\title{
TECHNICAL ASSOCIATION OF THE PULP AND PAPER INDUSTRY
}

\section{COLLABORATIVE REFERENCE PROGRAM FOR PAPER}

REPORT NO. 58G

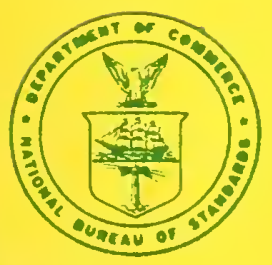

U.S. DEPARTMENT OF COMMERCE National Bureau of Standards

$=Q C$

100

.456

$79-1374$

1979

C. 2 


\section{TAPPI Paper and Board ( 6 times per year)}

Bursting strength

Tearing strength

Tensile breaking strength

Elongation to break

Tensile energy absorption

Folding endurance

Stiffness

Air resistance

Grammage
Smoothness

Surface pick strength

$\mathrm{K} \& \mathrm{~N}$ ink absorption

$\mathrm{pH}$

Opacity

Blue reflectance (brightness)

Specular gloss, $75^{\circ}$

Thickness

Concora (flat crush)

Ring crush

\section{FKBG-API Containerboard (48 times per year)}

Mullen burst of linerboard

Concora test of medium

MCCA Color and Appearance (4 times per year)

Gloss at $60^{\circ}$

Color and color difference

CTS Rubber ( 4 times per year)

Tensile strength, ultimate elongation and tensile stress

Hardness

Mooney viscosity

Vulcanization properties

\section{CTS Thermal Insulation Materials (2 times per year)}

19 test methods for thermal insulation materials covering: thermal properties; strength properties; dimensions, stability, and density properties; fire properties; and properties of vapor barriers

\section{ASTM Cement (2 times per year)}

Chemical (11 chemical components)

Physical (8 characteristics)

\section{AASHTO Bituminous}

Asphalt cement ( 2 times per year)

Cutbacks (once a year)

NBS Collaborative Reference Programs

A05 Technology Building

National Bureau of Standards

Washington, DC 20234 
TECHNICAL ASSOCIATION OF THE

PULP AND PAPER INDUSTRY

\title{
COLLABORATIVE REFERENCE PROGRAM FOR PAPER
}

Report No. 58G

\author{
R. G. Powell \\ TAPPI-NBS Research Associate \\ Collaborative Testing Services, Inc. \\ J. Horlick \\ Office of Testing Laboratory Evaluation Technology \\ Office of Engineering Standards \\ National Engineering Laboratory
}

NBSIR 79-1374

U. S. DEPARTMENT OF COMMERCE

National Bureau of Standards 


\section{INTRODUCTION}

Reports 585 and 586 comprise the fourth set of reports for the 78-79 program y ar. Participants in tests which involve strength properties of paper will receive only the S report; those in tests which measure other croperties will receive only the G report.

Notes and comments to individual laboratories and "Best Values" applicable to a paricular method are given following Table 1 for each method. See page 4 of this report for an explanation of "Best values". Please do not confuse these Best Values 1 th provisional values included with the samples to detect serious discrepancies at the $t$ ine of test. NBS results, identified as 1502 in the optical tests, are included on some or the tables.

If there are any questions on the notes, the analyses, or the reports in general, contact Robert G. Powell or Jeffrey Horlick on 301/921-2946.

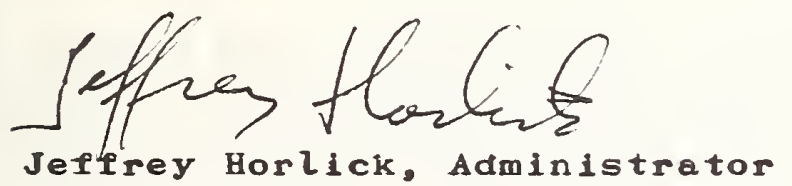

NBS-TAPPI Collaborative Reference Program Office of Testing Laboratory Evaluation Technology 


\section{BACKGROUND AND PURPOSE}

In 1969, the National Bureau of Standards and the Technical Assocjation of the Pulp and Paper Industry established a collaborative reference program to provide a participating laboratory with a means to check perlodically the level and uniformity of its testing in comparison with that of other laboratories.

The interchange of paper and board products and of the raw materials for these products requires agreement among raw material suppliers, paper and board producers, converters, distributors, retallers, commercial testing laboratorles, user organizations and the ultimate consumer as to the meaning of test results, an agreement that cannot be achieved without accurate and precise testing. This program is designed to help assure agreement.

\section{HOW THE PROGRAM WORKS}

Participants Select the Tests in which they wish to participate. This choice is made on joining the program, but additional tests may be added at any time. Also new participants may enter the program at any time.

Test Samples are Distributed Bimonthly; 1.e. every 2 months.

Provisional Values are Provided with the Samples for one or both of the test levels, depending on method. The provisional values permit serlous discrepancles to be detected without delay. (It is left to the discretion of the laboratory supervisor as to whether these values should be known to the operator.)

Each Participant Tests the Samples, following instructions provided for each test method. The full check on a single instrument should normally take no more than 30 minutes. The test results are then sent to NBS for analysis. The participant is also asked to report other Information relevant to an accurate analysis, such as test conditions and the instruments used.

Industry Means, Best Values and Other Statistics are developed from the data by NBS. The best values are estimates based on a careful examination of all data, both current and past, with special attention to results obtained by the National Bureau of Standards and other recognized reference laboratories in this and other countries.

A Quick Report is Prepared for each participating laboratory reporting data on time. This report shows the industry mean values, and the deviations of the laboratory's results from these values for each test method.

A Longer Summary Report, Showing the Data from all Participants, is also prepared. In the summary report, of which this report is an example, each laboratory is identifled by a code number so that the information is maintained on a confidential basis. However, instruments are identified by type so participants can compare their results with those obtalned on similar instruments of different manufacture. This report includes test averages, best values and standard deviations for individual participants and for the group as a whole. A participant should be able to readily determine the level and variability of his results in comparison with those of the other laboratories.

Repeatability and Reproducibllity Statements such as Contained in ASTM, TAPPI and ISO Standards are included at the end of the report. Participants can check their performance level against the precision statement given in the test method or specification. 
TABLE AF CONTENTS

Introduction

Description of Program

Metric Conversion Table

Key to Tables and Graphs

40-1 Alr Resistance, Gurley $\theta_{i l}$ type

40-2 Air Resistance, Shepfield type

4-1 Air Resistance, Gurley Mercury type

44-1 Smoothness, Parker Printsurp

45-1 Smoothness, Sheffield type

45-2 Smoothness, Bekk type

47-1 Snoothness, Bendtsen type

56-1 K \& N Ink Absorption

57-1 pH, Cold Extraction

57-2 pH, Hot Extraction

o $0-1$ Gpacity. White ( 89\%) Backing

60-2 Hpacity, Paper Backing, B \& I type

00-3 Opacity, Paper Backine. Elrepho type

65-1 Blue Reflectance (Brightness). Directional

65-2 Blue Reflectance, Diffuse, Elrepho (Gloss Irap)

05-3 Hiue Reflectance, Diffuse, Elrepho (No Gloss Trap)

75-1 Specular Glose, 75 degree

90-1 Thickness (Caliper)

95-1 Grammage (Basis Weight)

Summary

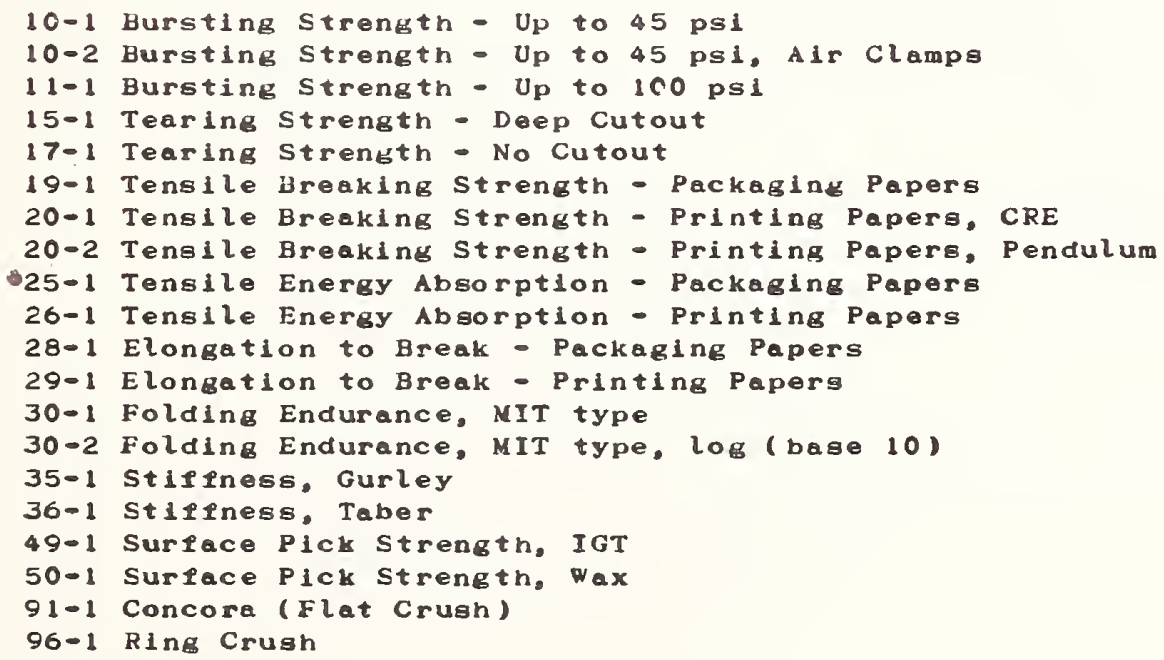


TABLE OF CONVERSION FACTCRS TO METRIC (SI) UNITS

Physical

Quantity

Bursting strength

Tearing strength

Tensile strength

Tensile energy absorption

Bending stiffness

Flat-crush strength (Concora)

Ring-crush (TAPPI)

(ISO)

Thickness
To Convert

From

psi

$\mathrm{kg} / \mathrm{cm}^{2}$

bar

g

1b/in.

$1 \mathrm{~b} / 0.5 \mathrm{in}$.

$1 \mathrm{~b} / 15 \mathrm{~mm}$

$\mathrm{kg} / 15 \mathrm{~mm}$

$\mathrm{kg} / 25 \mathrm{~mm}$

$\mathrm{kg} / \mathrm{mm}$

$f t-1 b / f t^{2}$

in. $-1 \mathrm{~b} / \mathrm{in}$. $^{2}$

$\mathrm{kg}-\mathrm{m} / \mathrm{m}^{2}$

$\mathrm{g} \cdot \mathrm{cm}$

$1 b$

$1 \mathrm{~b}$

$1 \mathrm{~b} / 6.00$ in.

mil
To

$\mathrm{kPa}$

$\mathrm{kPa}$

$\mathrm{kPa}$

$\mathrm{mN}$

$\mathrm{kN} / \mathrm{m}$

$\mathrm{kN} / \mathrm{m}$

$\mathrm{kN} / \mathrm{m}$

$\mathrm{kN} / \mathrm{m}$

$\mathrm{kN} / \mathrm{m}$

$\mathrm{kN} / \mathrm{m}$

$\mathrm{J} / \mathrm{m}^{2}$

$\mathrm{J} / \mathrm{m}^{2}$

$\mathrm{J} / \mathrm{m}^{2}$

$\mu \mathrm{N} \cdot \mathrm{m}$

$\mathrm{N}$

$\mathrm{N}$

$\mathrm{kN} / \mathrm{m}$

$\mu \mathrm{m}$
Multiply

by

6.895

98.07

100.00

9.807

.1751

.3502

.2965

.6538

.3923

9.807

14.59

175.1

9.807

98.07

4.448

4.448

0.0292

25.40 


\author{
MEAN - \\ GRAND MEAN - \\ (GR. MEAN)
}

SD OF MEANS -

(SD MEANS)

DEV -

N. DEV -

SDR -

AVERAGE SDR -

R. SDR -
The average of individual TEST DETERMINATIONS, The number of TEST DETERMINATIONS in the mean is given in the upper right corner of the first table (TEST D.) and again at the bottom of this table.

The average of the individual laboratory MEANS, excluding laboratories flagged (see column F) with an $X$, 非, or + . The GRAND MEAN is given in US customary units and, where applicable, in SI metric units.

The standard deviation of the laboratory MEANS about the GRAND MEAN; an index of the amonglaboratory precision.

The deviation or difference of the laboratory MEAN from the GRAND MEAN.

The normal deviate or ratio of the DEV to the SD OF MEANS; an indication of the degree of divergence of the laboratory MEAN from the GRAND MEAN. A N. DEV of more than 2 or less than -2 may indicate that the participant is not following the procedure considered standard for this analysis.

The standard deviation of repeated measurements; that is, of individual test determinations about their MEAN.

The average of the individual laboratory SDR's; an index of the within-laboratory precision of repeated measurements.

The relative standard deviation of repeated measurements; that is, the ratio of the SDR to the AVERAGE SDR; an indication of the ability of a participant to repeat his measurements relative to the average ability. The greater the number of TEST DETERMINATIONS the closer the R. SDR should be to unity. If $R$. SDR is outside the limits given below, the participant may not be following the procedure considered standard for this analysis: 
No. of test

Determinations

3

5

8

10

15

20

25
Lower limit

for R. SDR

0.09

0.27

0.40

0.46

0.56

0.61

0.65
Upper limit

for R. SDR

2.58

2.06

1.77

1.67

1.53

1.45

1.39
VAR -

F -

M -

X -

* -

S -

0 -

COORDINATES -

Code for instrument type or variation in condition, see second table.

Flag, with following meaning:

Excluded from grand means because VAR nonstandard for this analysis.

Excluded because data were not understood or because of a non-coded variation reported by the laboratory. (See NOTES following Table 1 for each method).

Excluded because data for one sample are missing.

Excluded because plotted point would fall outside of the $99 \%$ error ellipse, (see below for explanation of Graph).

Included in grand means but plotted point falls outside of the $95 \%$ error ellipse. The participants should take this as a warning to reexamine his testing procedure.

Included in grand mean but only after omission of one or more 'wild' values; that is, test determinations more than 3 times AVERAGE SDR from the laboratory's MEAN. Not more than 20\% of the test determination may be excluded in this manner without rejecting the laboratory. Included in grand mean and inside $95 \%$ error ellipse.

Distances along major and minor axes of error ellipse. If special additive or concurrent model of the measuring process applies to this method, the distance along the minor axis represents the random error within a laboratory while that along the major axis also includes a systematic laboratory component of error. 
95\% ELLIPSE -

AVG R. SDR -

$\underline{\text { Graph - }}$
Lengths of the major and minor axes of the ellipse and the angle that the major axis makes with the horizontal axis.

Average of the R. SDR for the two samples; an indication of the laboratory's precision of repeated measurements.

For each laboratory the MEAN for the second sample is plotted against the MEAN for the first sample, with each point representing a laboratory. The horizontal and vertical lines are the GRAND MEANS. The dashed line is drawn at $45^{\circ}$. The solid sloping line, which may or may not lie close to the $45^{\circ}$ line, is along the major axis of the error ellipse. The ellipse is drawn so that, on the average, it will include $95 \%$ of the points representing the laboratories.

Plotted symbols are as explained above (under $F)$, except that an ' $S$ ' is plotted as an ' $O$ '. A participant whose plotted point falls outside of the ellipse should carefully reexamine the testing procedure he is following.

The graph is plotted with an ellipse when there are 20 or more laboratories in the analysis. When there are 10 through 19 laboratories in the analysis the graph is plotted but the ellipse is omitted. When there are fewer than 10 laboratories retained in the analysis the graph is not plotted.

The International System of Units (SI) is used on the plots wherever possible to aid participants in familiarizing themselves with SI. Grand means in SI units are given at the top of the plot, and supplementary scales in SI units are drawn along the axes allowing the reader to compare means and variability in common units and SI units for the same data. 


\begin{abstract}
Summary -
(At end of report)

REPL CRP -

REPL TAPPI -

REPEAT -

REPROD -

In addition to several quantities already defined above,the summary shows the following values for each test method:

The number of replicate test determinations used in this Collaborative Reference Program.

The number of replicate test determinations in a test result required by the applicable TAPPI Standard or assumed here if there is no TAPPI Standard. This quantity is needed in the computation of TAPPI repeatability and reproducibility from the SD OF MEANS and the AVER SDR. See TAPPI Standard T1206 for definitions and computations.

TAPPI repeatability, a measure of the withinlaboratory precision of a test result.

TAPPI reproducibility, a measure of the betweenlaboratory precision of a test result.
\end{abstract}

Best values -
Given at the end of Table 1 for each method for which sufficient information is available. These best values are estimates based on a careful examination of all data, both current and past, with special attention to results obtained by the National Bureau of Standards and other recognized reference laboratories in this and other countries. All participants using equipment that is standard for the analysis should be able to achieve results within the plus-minus ( $(+)$ limits, when these are shown along with the best values. 


\begin{tabular}{|c|c|c|c|c|c|c|c|c|c|c|c|c|c|}
\hline & SAMPLE & \multicolumn{4}{|c|}{ PRIN IING } & SAMPLE & \multicolumn{4}{|c|}{ PR I N T I NG } & \multirow[t]{2}{*}{ TEST } & \multirow[t]{2}{*}{$D_{\bullet} \cdot$} & 10 \\
\hline LAB & $\mathrm{R} 21$ & $103 \mathrm{GLAMS}$ & PEF $S$ & SCUARE ME & & 843 & $60 \mathrm{GR}$ & PER & SUDARE Y & & & & \\
\hline CEDE & MEAN & DEV & N. DEV & $S D R$ & $R_{\bullet} S D R$ & MEAN & DEV & $N, D E V$ & SDR & $R \cdot S D R$ & VAR & $F$ & LAlS \\
\hline L 100 & 56.6 & 5.4 & 1.78 & $7 \cdot 3$ & 1.70 & 17.0 & .0 & .73 & 1.3 & 1.11 & 400 & 9 & L 100 \\
\hline L106 & 40.5 & -10.7 & -3.56 & 1.2 & .28 & 12.6 & -3.8 & -4.52 & .7 & .59 & $40 D$ & $\mathrm{x}$ & L106 \\
\hline L 107 & 52.6 & $1 \cdot 3$ & .44 & 5,8 & 1.35 & $17 \cdot 1$ & .7 & .89 & .6 & .49 & $4 C D$ & $\boldsymbol{\theta}$ & $L_{1} 07$ \\
\hline L1 21 & 50.9 & .04 & -.12 & 4.2 & .97 & 15.7 & -.7 & -.80 & .7 & .58 & $4 O D$ & $\boldsymbol{\theta}$ & L121 \\
\hline L122 & 50.0 & -1.2 & -.40 & 3.2 & .74 & 16.0 &. .4 & -.43 & 1.7 & 1.45 & $40 D$ & क & L1 22 \\
\hline 1123 & 52.1 & - 8 & .27 & 4.1 & .97 & 15.7 & -.7 & .80 & 1.4 & 1.22 & $4 O D$ & $\theta$ & L 123 \\
\hline LI $24 G$ & $48 \cdot 8$ & -2.4 & -.81 & 2.9 & .67 & 16.0 & -.4 & .044 & . 8 & .66 & $\triangle O D$ & $A$ & L124G \\
\hline L125 & 50.0 & -1.3 & -.42 & 3.3 & .77 & 16.6 & .2 & .27 & 1.3 & 1.07 & $4 O D$ & $\theta$ & L 125 \\
\hline L1 28 & 47.6 & -3.6 & -1.21 & 4.4 & 1.03 & 16.4 & .0 & .03 & 1.5 & 1.27 & $40 D$ & $\theta$ & L128 \\
\hline L141 & 52.0 & . 8 & .25 & 3. 7 & .87 & 17.1 & - 8 & .90 & 1.0 & .83 & $40 D$ & $\theta$ & L 141 \\
\hline$L 148$ & 53.6 & $2 \cdot 4$ & .78 & 4.3 & 1.01 & 16.6 & - 2 & .24 & 1.4 & 1.19 & $40 D$ & $A$ & L 148 \\
\hline L1 53 & 48.4 & $-2 \cdot 8$ & -.94 & $5 \cdot 9$ & 1.38 & 16.9 & .5 & .62 & 1.4 & 1.17 & 400 & $\theta$ & L 153 \\
\hline L158 & 46.9 & $-4 \cdot 3$ & -1.44 & 4.9 & 1.14 & 15.8 & -.0 & -.65 & 1.3 & 1.11 & $4 O D$ & $\theta$ & L158 \\
\hline L159 & 50.9 & -.3 &. .10 & 1.7 & .39 & 15.9 &. .5 & -.62 & .8 & .71 & $4 O D$ & $\theta$ & L159 \\
\hline L1 63 & 52.3 & 1.0 & .34 & 5.8 & 1.36 & 16.6 & - 2 & . 29 & - 8 & .69 & $40 D$ & 9 & L 163 \\
\hline L 166 & $51 \cdot 0$ & $\cdot 3$ & .11 & 4.1 & .96 & 16.5 & $\cdot 2$ & .18 & $1 \cdot 3$ & 1.07 & $4 O D$ & $\boldsymbol{\sigma}$ & L166 \\
\hline L174 & 51.4 & $\cdot 2$ & . C5 & $4 \cdot 8$ & 1.12 & 16.6 & - 2 & .22 & 1.2 & 1.02 & $40 D$ & $\theta$ & L174 \\
\hline I $182 G$ & 50.9 & -.3 & -011 & 7.1 & 1.66 & 16.4 & -.0 & -.02 & $1 \cdot 6$ & $1 \cdot 38$ & $4 O D$ & $\boldsymbol{\theta}$ & L $182 G$ \\
\hline L183 & 56.7 & 5.5 & 1.81 & 4.9 & 1.14 & 17.3 & 1.0 & 1.15 & 1.2 & 1.04 & $4 O D$ & $\theta$ & L 183 \\
\hline LIS OC & 52.7 & 1.5 & .49 & 5.6 & 1.32 & 16.4 & .0 & .01 & 1. 7 & 1.43 & $40 \mathrm{D}$ & $A$ & L190C \\
\hline L. $190 \mathrm{~K}$ & 54.4 & 3.2 & $1 \cdot 05$ & 6.7 & 1.57 & 16.1 & -.3 & -.31 & 1.2 & 1.05 & $4 O D$ & + & L $190 \mathrm{r}$ \\
\hline L21 2 & 47.4 & -3.8 & -1.27 & 5.8 & 1.36 & 14.5 & -1.9 & -2.23 & - 8 & .66 & $40 D$ & $\theta$ & L2 12 \\
\hline L219 & 47.4 & -3.8 & -1.26 & 2.9 & .68 & 15.9 & -.5 & $\because 60$ & 1.0 & .85 & $4 O D$ & A & L219 \\
\hline 1223 & 53.6 & 2.4 & .78 & 4.1 & .96 & $17 \cdot 6$ & 1.2 & 1.43 & 1.3 & 1.10 & $40 D$ & $\boldsymbol{G}$ & L 223 \\
\hline L230G & 59.7 & e. 5 & 2.81 & 5.5 & 1.28 & 18.3 & 1.9 & 2.30 & 1.3 & 1.06 & $4 O D$ & * & L230G \\
\hline L 232 & 43.0 & $-8 \cdot 3$ & -2.75 & 7.8 & 1.83 & 14.6 & -1.8 & -2.13 & 1.1 & .93 & $40 \mathrm{D}$ & * & L232 \\
\hline L.236 & 52.7 & 1.5 & .48 & 5.4 & 1.26 & 17.0 & .6 & - 76 & 1.0 & .83 & $40 D$ & $\theta$ & L230 \\
\hline L23 8A & 51.7 & - 5 & .15 & 2.5 & .58 & 17.0 & .6 & .76 & 1.0 & - 86 & $4 O D$ & e & L 238A \\
\hline L241 & 47.8 & -3.4 & -1.14 & 3.8 & - 88 & 15.7 & -.7 &. .81 & .9 & .80 & $4 C D$ & $\theta$ & L241 \\
\hline L24 2 & 50.0 & -1.2 &. .41 & 1.5 & .35 & 15.3 & -1.1 & -1.32 & 1.2 & 1.05 & $4 O D$ & 9 & L242 \\
\hline L $243 G$ & 49.6 & -1.6 & -.54 & 4.8 & 1.13 & $15 \cdot 7$ & $\because 7$ & -.85 & - 8 & .70 & $40 \mathrm{D}$ & $\theta$ & L243G \\
\hline L25 4 & 52.2 & 1.0 & .32 & 3.8 & - 89 & $17 \cdot 2$ & - 8 & .98 & - 9 & .78 & $40 D$ & $\theta$ & L254 \\
\hline L259 & 47.9 & $-3 \cdot 3$ & -1.11 & 3. 1 & .73 & 14.8 & -1.5 & -1.83 & 1.1 & .92 & $40 D$ & $\boldsymbol{b}$ & L259 \\
\hline 1201 & 51.8 & . 6 & .19 & 5.6 & 1.30 & 16.1 & -.3 & -.31 & 1.4 & 1.19 & $40 D$ & 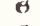 & L 261 \\
\hline L262G & 50.4 & .8 & -.27 & 1.8 & .43 & 16.6 & .2 & .22 & 1.2 & 1.05 & $4 O D$ & $\boldsymbol{A}$ & L $262 G$ \\
\hline L205 & 48.5 & -2.8 & -.91 & 4.7 & 1.11 & 15.9 & -.5 & -.62 & 1.1 & .92 & $4 O D$ & $\theta$ & L265 \\
\hline L278 & 51.0 & -.3 & -.09 & 3.8 & -88 & $1 \epsilon \cdot 2$ & -.2 & -.19 & 1.4 & 1.16 & $40 D$ & $A$ & L2 278 \\
\hline L285 & 52.4 & 1.2 & .40 & 5.4 & 1.26 & 16.7 & $\cdot 3$ & .36 & 1.2 & 1.02 & $4 O D$ & $\theta$ & L2 85 \\
\hline 1301 & 50.1 & -1.2 & -.38 & 4.3 & 1.00 & 16.3 & -.0 & -.06 & 1.3 & 1.08 & 400 & $\theta$ & 1301 \\
\hline$L 308$ & 50.9 & -.3 &. .11 & 3. 7 & .86 & 16.6 & .2 & .29 & $1 \cdot 3$ & 1.14 & $4 O D$ & $\theta$ & L.308 \\
\hline L320 & 46.8 & -4.4 & -1.47 & 2.7 & .63 & 15.6 & -.8 & .93 & 1.8 & 1.55 & $4 O D$ & $\theta$ & L320 \\
\hline L321 & 46.0 & -5.2 & -1.74 & 3.6 & .85 & 12.8 & -3.6 & -4.28 & .6 & .53 & $40 D$ & $\mathbf{x}$ & I321 \\
\hline L324 & 50.4 &. .5 & -.28 & 4.2 & .98 & 16.4 & . 1 & .06 & 1.0 & .81 & $4 O D$ & $\theta$ & L324 \\
\hline L326 & 54.3 & 3.1 & 1.03 & $7 \cdot 1$ & 1.65 & 18.4 & 2.0 & 2.40 & 1.0 & .88 & $40 D$ & $\star$ & L326 \\
\hline$L \overline{3} 28$ & 50.8 & -.4 & -.14 & 4.8 & 1.12 & 16.7 & .3 & .35 & 1.4 & 1.20 & $4 O D$ & $\theta$ & $\mathrm{L} 328$ \\
\hline L339 & 40.4 & -10.8 & -3.60 & 5.0 & 1.18 & 12.1 & $-4 \cdot 3$ & -5.10 & 1.0 & .86 & $40 D$ & $\mathbf{X}$ & L339 \\
\hline 1344 & 47.4 & -3.8 & -1.27 & 4.8 & 1.12 & 15.6 & -.8 & -.98 & - 9 & .76 & $4 O D$ & $\theta$ & 1344 \\
\hline 1376 & 53.6 & 2.4 & .78 & 5.0 & 1.17 & 17.0 & .6 & .70 & 1.4 & 1.17 & $40 D$ & $\theta$ & L376 \\
\hline L380 & 50.6 & -.6 & -.21 & 2.1 & .48 & 15.6 & -.8 &. .93 & 1.3 & 1.07 & $4 O D$ & $\theta$ & L380 \\
\hline L388 & 59.5 & 8.2 & 2.73 & 5.0 & 1.16 & 17.2 & .9 & 1.03 & 1.6 & 1.39 & $4 O D$ & $*$ & L388 \\
\hline 1394 & 54.4 & 3.2 & 1.05 & 5.1 & 1.19 & 17.6 & 1.2 & 1.43 & $1 \cdot 3$ & 1.13 & 4OD & G & L394 \\
\hline $1396 \mathrm{~N}$ & 48.5 & -2.7 & -.89 & 5.5 & 1.25 & 46.5 & 30.1 & 35.99 & 2.3 & 1.94 & $40 D$ & $*$ & L396M \\
\hline L56 7 & 53.1 & 1.9 & .62 & 3.6 & .85 & 16.7 & .3 & .37 & 1.6 & $1 \cdot 37$ & 400 & $\boldsymbol{\theta}$ & L567 \\
\hline L576 & 51.5 & $\cdot 2$ & .07 & 4.0 & .54 & 15.1 & $-1,3$ & -1.52 & 1.1 & .92 & $40 D$ & $\theta$ & 1576 \\
\hline L585 & 53.7 & 2.5 & .82 & 5.3 & 1.23 & 17.2 & .8 & .96 & 1.4 & 1.18 & $4 O D$ & 0 & L5 85 \\
\hline L5ऽ 7 & 50.5 & -.7 &. .24 & 3. 1 & .72 & 16.3 & -1 & -.09 & 1.8 & 1.55 & $40 D$ & $\theta$ & L597 \\
\hline L604 & 51.3 & - 1 & .02 & 5.1 & 1.19 & 16.1 & -.3 & -.33 & .6 & .48 & 400 & $\theta$ & L604 \\
\hline 2616 & 52.3 & 1.1 & .37 & 3.3 & .77 & 17.7 & 1.3 & 1.54 & $\cdot 3$ & .24 & $4 O D$ & $\sigma$ & 2616 \\
\hline 2651 & 45.4 & $-1 \cdot 8$ & -.61 & 3.8 & .88 & 15.0 & -1.4 & -1.65 & 1.1 & .89 & 4OD & $\sigma$ & L651 \\
\hline
\end{tabular}

GR. MEAN - 51.2 GURLEY UNITS GRAND MEAN $=16.4$ GURLEY UNITS SD MTANS = 300 GURLEY UNIT $\triangle$ VERAGE SDR .
GRAND MEAN $=16.4$ GURLEY UNITS 4.3 GURLEY UNITS AVERAGE SDR
TEST DETERYINATIGNS = 10 55 LABS IN GRAND MEAN 1.2 GURLEY UNITS

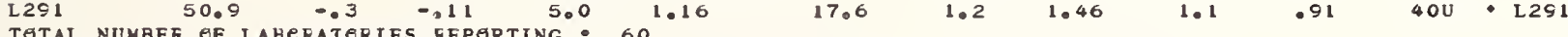
Best values: K21 $51.0+4.1$ Gurley units 


\begin{tabular}{|c|c|c|c|c|c|c|c|c|c|c|c|c|c|}
\hline \multirow{2}{*}{$\begin{array}{l}\text {LAB} \\
\text { CबLE }\end{array}$} & \multirow[b]{2}{*}{$\mathbf{F}$} & \multicolumn{2}{|c|}{ MEANS } & \multicolumn{2}{|c|}{ CEORDINATES } & $A \nabla G$ & \multirow[b]{2}{*}{$V_{A R}$} & \multirow{2}{*}{\multicolumn{2}{|c|}{ PREPERTY $=-$ TEST }} & \multirow{2}{*}{\multicolumn{3}{|c|}{ ST INSTRUMENT $--C$ CENDITI TN }} & \\
\hline & & K2 1 & 543 & HAJER & MINER & R. SDK & & & & & & & Ns \\
\hline L33G & $\mathbf{x}$ & 40.4 & 12.1 & $-11 \cdot 5$ & -1.9 & 1.02 & $40 \mathrm{D}$ & $\mathbf{A I R}$ & RES IST ANCE & GURLEY & DENSOMETER & - $\boldsymbol{\theta I L}$ & FIETAIIEN \\
\hline L106 & $\mathbf{x}$ & 40.5 & 12.6 & $-11 \cdot 3$ & -1.5 & .43 & $40 \mathrm{D}$ & $\mathbf{A I R}$ & RES I ST ANCE, & GURLEY & DENSOMETER & - $\boldsymbol{H I L}$ & FLETATIAN \\
\hline L232 & - & 3.0 & $14 \cdot 6$ & -8.5 & .0 & 1.38 & $40 \mathrm{D}$ & $\mathbf{A I R}$ & RESIST ANCE, & GURLEY & DENSOMETER & - $\theta \mathbf{I L}$ & FLETATION \\
\hline 1321 & $\mathbf{x}$ & 46.0 & 12.8 & -5.9 & -2.4 & .69 & $40 \mathrm{D}$ & $A I R$ & RESISTANCE, & GUR LEY & DENSOMETER & - $\boldsymbol{\theta I} \mathbf{L}$ & FLETATIGN \\
\hline 2320 & $\theta$ & $4 \epsilon .8$ & 15.6 & $-4 \cdot 5$ & $\cdot 2$ & 1.09 & $40 \mathrm{D}$ & AIR & RES IST ANCE, & GURLEY & DENSEMETER & $-\theta I L$ & FLAT $\triangle I$ I \\
\hline L158 & a & 46.9 & 15.8 & -4.4 & .3 & 1.12 & $4 O D$ & AIR & RESISI ANCE, & G UR LEY & DENSOMETER & $-\theta I \mathbf{L}$ & FLETAIIUN \\
\hline 1344 & $\theta$ & 47.4 & 15.6 & -3.9 & $\because 0$ & .94 & $4 O D$ & $A I R$ & RESISTANCE, & GURLEY & DENSOMETER & - $\boldsymbol{\theta I L}$ & FLEIATIEN \\
\hline L212 & $\boldsymbol{\theta}$ & 47.4 & $14 \cdot 5$ & -4.1 & -1.0 & 1.01 & $40 \mathrm{D}$ & $\Delta I R$ & RESISTANCE, & GURLEY & DENSOMETER & - OIL & FLETATION \\
\hline L219 & $\boldsymbol{\theta}$ & 47.4 & 15.9 & -3.8 & .3 & .76 & $4 O D$ & $A I R$ & RESISTANCE, & GURLEY & DENSEMETER & - $\boldsymbol{\theta I L}$ & FLETATIEN \\
\hline 1128 & $\theta$ & 47.6 & 16.4 & -3.6 & .8 & 1.15 & $40 D$ & AI R & RESISTANCE, & GURLEY & DENSOMETER & - OIL & FLETATIEN \\
\hline L241 & $\theta$ & $47 \cdot 8$ & 15.7 & -3.5 & .0 & .84 & $4 O D$ & AI R & RESISTANCE, & GURLEY & DEN SEMETER & $-\boldsymbol{\theta I} \mathbf{L}$ & PLETATIEN \\
\hline L259 & $\boldsymbol{\theta}$ & 47.9 & 14.8 & -3.6 & .08 & .82 & $4 O D$ & $\Delta I R$ & RESI ST ANCE, & GUR LEY & DENSOMETER & $-\theta I L$ & FLETATIEN \\
\hline L153 & g & 48.4 & 16.9 & -2.7 & 1. 1 & 1.27 & $40 \mathrm{D}$ & $A I R$ & RESI STANCE, & GURLEY & DENSEMETER & - $\boldsymbol{\theta I L}$ & FLETATION \\
\hline 2265 & A & 48.5 & 15.9 & $-2 \cdot 8$ & .1 & 1.01 & $4 O D$ & $\Delta I R$ & RESISTANCE, & GUR LEY & DENSEMETER & - $\boldsymbol{\theta I L}$ & FLOTATION \\
\hline L396M & $*$ & 48.5 & 46.5 & 3.6 & 30.0 & 1.62 & $40 \mathrm{D}$ & AI R & RESIST $\triangle N C E$ & GURLEY & DENSEXETER & - AIL & FLETATIEN \\
\hline L124G & $\boldsymbol{\theta}$ & 48.8 & 16.0 & -2.5 & .1 & .06 & $40 \mathrm{D}$ & $\Delta I R$ & RESIST ANCE, & GURLEY & DENSOMETER & - $\boldsymbol{\theta I L}$ & FLETATIGN \\
\hline 1651 & $\boldsymbol{\theta}$ & 49.4 & 15.0 & $-2 \cdot 1$ & -1.0 & .89 & $\triangle O D$ & AIR & RESISTANCE, & GUEIEY & DENSEMEIER & - GIL & FLETATION \\
\hline L243G & $\theta$ & 49.6 & 15,7 & -1.7 & .4 & .91 & $4 O D$ & AI R & RESI ST ANCE, & GURLEY & DENSEMETER & - OIL & FLETATIEN \\
\hline L125 & $\theta$ & 50.0 & 16.6 & $-1 \cdot 2$ & .5 & .92 & $\triangle O D$ & AIR & RESISTANCE. & GURLEY & DENSEMEIER & - $\boldsymbol{\theta I L}$ & FLETATIEN \\
\hline L242 & e & 50.0 & 15.3 & -1.4 & .08 & .70 & $40 D$ & AIR & RESISTANCE, & GURLEY & DENSEMETER & $=\operatorname{GIL}$ & FLETATIGN \\
\hline 1122 & $\boldsymbol{\theta}$ & $5 \mathrm{c} .0$ & 16.0 & $-1 \cdot 3$ & -1 & 1.09 & $40 D$ & A I R & RES IST ANCE, & GURLEY & DENSOMETER & - $\boldsymbol{\text { CIL }}$ & FLETATIEN \\
\hline 1301 & $A$ & 50.1 & $16 \cdot 3$ & -1.1 & .2 & 1.04 & $40 D$ & AI R & RES I ST ANCE & GURLEY & DENSOMETER & $=\theta I L$ & FLETATIEN \\
\hline L324 & $\boldsymbol{\theta}$ & 50.4 & 16.4 & .08 & .2 & .90 & $40 D$ & AIR & RESIST ANCE, & GURLEY & DENSEMETER & - OIL & FLETATIEN \\
\hline L262G & $\theta$ & 50.4 & 16.6 & -.8 & .3 & .74 & $40 D$ & $\mathbf{A I} \mathbf{R}$ & RESISTANCE, & GUR IEY & DENSOMETER & - OIL & FLOT ATION \\
\hline L5S7 & $\theta$ & 50.5 & 16.3 & -.7 & .1 & 1.13 & $40 D$ & AI R & RESIST ANCE & GUR LEY & DENSOMETEK & - OIL & FLOTATIEN \\
\hline L380 & $\theta$ & 50.6 & 15.6 &. .8 & -.6 & .78 & $\triangle O D$ & $\mathbf{A I R}$ & RESISTANCE, & GURLEY & DENSEMETER & - $\boldsymbol{\theta I L}$ & FLETATI GN \\
\hline L328 & e & 50.8 & 16.7 & -.4 & .4 & 1.16 & $4 O D$ & AIR & RESISTANCE, & GURLEY & DENSEMETER & - $\theta I L$ & FLOTATIEN \\
\hline L121 & $\theta$ & 50.9 & 15.7 &. .5 & .06 & .77 & $40 \mathrm{D}$ & AI R & RESISTANCE, & GUKLEY & DENSOMET ER & - $\boldsymbol{\theta I L}$ & FLETATIEN \\
\hline L291 & - & 50.9 & 17.6 & .01 & $1 \cdot 3$ & 1.04 & 400 & AI R & RESI STANCE, & SHEFF I & ELD IN GURLE & EY UNI? & Is \\
\hline 1308 & $\theta$ & 50.9 & 16.6 &. .3 & .3 & 1.00 & $4 O D$ & $A I R$ & RESISI $\triangle$ NCE & G UR LEY & DENSEMEIER & $-\boldsymbol{\theta I L}$ & FLOTATION \\
\hline L $182 G$ & $\boldsymbol{\theta}$ & 50.9 & 10.4 & -.3 & $\cdot 1$ & 1.52 & $40 D$ & AI R & RESI STANCE, & GORLEY & DENSEMETER & $-\theta I L$ & FLETATIEN \\
\hline L159 & $\boldsymbol{\theta}$ & 50.9 & 15.5 & -.4 & -.4 & .55 & $40 \mathrm{D}$ & AIR & RES IST ANCE, & GURLEY & DENSOMETER & - $\theta I L$ & FLETATIEN \\
\hline L278 & a & 51.0 & 16.2 & -.3 & .11 & 1.02 & $40 D$ & A I R & RESISTANCE, & GURLEY & DENSEMETER & - $\theta$ IL & FLETATIEN \\
\hline 1604 & A & $51 \cdot 3$ & 16.1 & .0 & -.3 & .84 & 400 & AIR & RESISTANCE, & GUR LEY & DENSEMETER & - $\boldsymbol{\theta I L}$ & FLETATIEN \\
\hline 1174 & $\theta$ & 51.4 & 16.6 & $\cdot 2$ & .1 & 1.07 & $\triangle O D$ & AIR & RESI STANCE, & GURLEY & DEN SEMETER & - $\boldsymbol{\theta I L}$ & FLETATIEN \\
\hline L576 & $\boldsymbol{\theta}$ & 51.5 & 15.1 & .0 & $-1 \cdot 3$ & .93 & $4 O D$ & A I R & RESISTANCE, & GURLEY & DENSOMETER & - OIL & FLETATIEN \\
\hline L166 & H & 51.6 & 16.5 & .3 & $\cdot 1$ & 1.02 & $40 D$ & $\Delta I R$ & RESISTANCE, & GURLEY & DENSOMETER & - AIL & FLETATIEN \\
\hline L238A & $\boldsymbol{\theta}$ & 51.7 & 17.0 & .6 & .5 & .72 & $40 \mathrm{D}$ & AIR & RESISTANCE, & GURLEY & DENSOMETER & - AIL & FLETATI GN \\
\hline L261 & a & 51.8 & 16.1 & .5 & .4 & 1.24 & $40 \mathrm{D}$ & $\Delta I R$ & RESISTANCE, & GURLEY & DENSOMETER & - OIL & FLETATIEN \\
\hline 1141 & $\theta$ & 52.0 & 17.1 & .9 & .6 & .85 & $40 D$ & AI R & RES I ST $\triangle N C E$ & GUR LEY & DENSEMETER & - AIL & FLETATIAN \\
\hline L123 & $\theta$ & 52.1 & $15 \cdot 7$ & .7 &. .8 & 1.09 & $40 \mathrm{D}$ & AIR & RES I STANCE, & GUP LEY & DENSEMETER & - OIL & FLETATION \\
\hline L254 & $\theta$ & 52.2 & 17.2 & 1.1 & .6 & .83 & $\triangle O D$ & $\Delta I R$ & RESISTANCE, & GURLEY & DENSOMETER & - $\theta \mathbf{L}$ & FLOTATION \\
\hline 1163 & $\theta$ & 52.3 & 16.6 & $1 \cdot 1$ & .0 & 1.02 & $\triangle O D$ & $A I R$ & RESI ST ANCE, & GUR LEY & DEN SOMETER & - $\boldsymbol{\theta I L}$ & FLETATION \\
\hline 2616 & $\theta$ & 52.3 & 17.7 & 1.4 & 1.0 & .50 & 4OD & $\mathbf{A I R}$ & RESISTANCE, & GURLEY & DENSEMETER & $-\theta I L$ & FLOTATIEN \\
\hline L285 & e & 52.4 & 16.7 & 1.2 & .0 & 1.14 & $40 \mathrm{D}$ & $\Delta I R$ & RESISIANCE, & GURLEY & DENSEMETER & - OIL & FLETATIEN \\
\hline 1107 & $\theta$ & 2.6 & 17.1 & 1.4 & .5 & .92 & $40 \mathrm{D}$ & AI R & RESISTANCE, & GUR LEY & DEN SAMETER & - OIL & TAT ION \\
\hline L236 & $\boldsymbol{\theta}$ & 52.7 & 17.0 & 1.6 & $\cdot 3$ & 1.05 & 400 & $\Delta I R$ & RESISTANCE, & GURLEY & DENSEMETER & - $\boldsymbol{\theta I L}$ & FLETATIEN \\
\hline L $190 \mathrm{C}$ & $\theta$ & 52.7 & 16.4 & 1.4 &. .3 & 1.37 & $40 \mathrm{D}$ & $\mathbf{A I} \mathbf{R}$ & RES ISTANCE, & GUR LEY & DENSEMETER & - AIL & FLETATIEN \\
\hline L567 & $\leftrightarrow$ & 53.1 & 16.7 & 1.9 & -1 & 1.11 & $40 D$ & $\mathbf{A I} R$ & RESISTANCE, & GURLEY & DENSEMETER & - भII & FLGTATIEN \\
\hline$L 376$ & 0 & 53.6 & 17.0 & 2.4 & $\cdot 1$ & 1.17 & $40 \mathrm{D}$ & A I R & RESISTANCE, & GUKLEY & DENSAMETEK & - $\theta I L$ & FLETAT IEN \\
\hline 2148 & $\boldsymbol{\theta}$ & & 16.6 & 2.4 & -.3 & 1.10 & $\triangle O D$ & $\Delta I R$ & RES I ST $\triangle N C E$, & GURLEY & DENSEMETER & - AII & PLET $\triangle$ I IGN \\
\hline L223 & $\boldsymbol{\theta}$ & 3.6 & 17.6 & $2 \cdot 6$ & .7 & 1.03 & $40 D$ & $A I R$ & RESISI $\triangle N C E$, & GURLEY & DENSAMETER & $-\theta I L$ & FLETATI $\sigma N$ \\
\hline L.585 & $\theta$ & 53.7 & 17.2 & 2.6 & $\cdot 3$ & 1.20 & $40 D$ & $A I R$ & RES IST $\triangle N C E$, & GURLEY & DENSOMETER & $-\theta I L$ & FLETATIEN \\
\hline 2326 & * & $54 \cdot 3$ & 18.4 & $3 \cdot 4$ & 1.3 & 1.27 & $4 O D$ & A IR & RESIST $A N C E$, & GURLEY & DENSOMETER & - $\theta I L$ & FLETATIEN \\
\hline L3S4 & e & $54 \cdot 4$ & 17.6 & $3 \cdot 3$ & .5 & 1.16 & $40 D$ & $\mathbf{A I} \mathbf{R}$ & RESISTANCE. & GURLEY & DENSEMETER & $-\theta I L$ & FLETATIEN \\
\hline L $190 R$ & $\boldsymbol{\theta}$ & 54.4 & 16.1 & 3.1 & -.9 & 1.31 & $40 \mathrm{D}$ & A I R & RESISTANCE, & GURLEY & DENSOMETER & - $\theta I L$ & FLOTATION \\
\hline L100 & $\boldsymbol{\theta}$ & 56.6 & 17.0 & 5.4 & -.5 & 1.40 & $4 O D$ & AI R & RESISTANCE, & GUR LEY & DEN SOMETER & $-\theta \mathbf{I L}$ & FLOTATIEN \\
\hline $\mathbf{L 1 8 3}$ & $\boldsymbol{\theta}$ & 56.7 & $17 \cdot 3$ & 5.5 & -.2 & 1.09 & $40 D$ & AIR & RES ISTANCE, & GURLEY & DENSEMETER & - $\theta I L$ & FLOTATION \\
\hline 1388 & 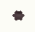 & 59.5 & 17.2 & 8.2 & -.9 & 1.27 & $4 O D$ & $\Delta \mathbf{I} \mathbf{R}$ & RESIST $\triangle N C E$, & GURLEY & DENSAMETER & - $\boldsymbol{\theta I L}$ & FLETAIION \\
\hline L230G & - & 59.7 & 18.3 & 8.7 & .1 & 1.17 & $4 O D$ & AIR & RES ISTANCE, & GURLEY & DENSEMEIER & $-\theta \mathbf{I} \mathbf{L}$ & FLOTATIGN \\
\hline & & $\begin{array}{l}51.2 \\
95 \%\end{array}$ & $\begin{array}{l}16.4 \\
\text { IPSE: }\end{array}$ & 7.8 & 1.4 & $\begin{array}{l}1.00 \\
\text { WITH }\end{array}$ & GAH & & DEGREES & & & & \\
\hline
\end{tabular}


AIR RESISTANCE, GURLEY

SAMPLE K21 $=51.2$ GURLEY UNITS SAMPLE K43 $=16.4$ GURLEY UNITS

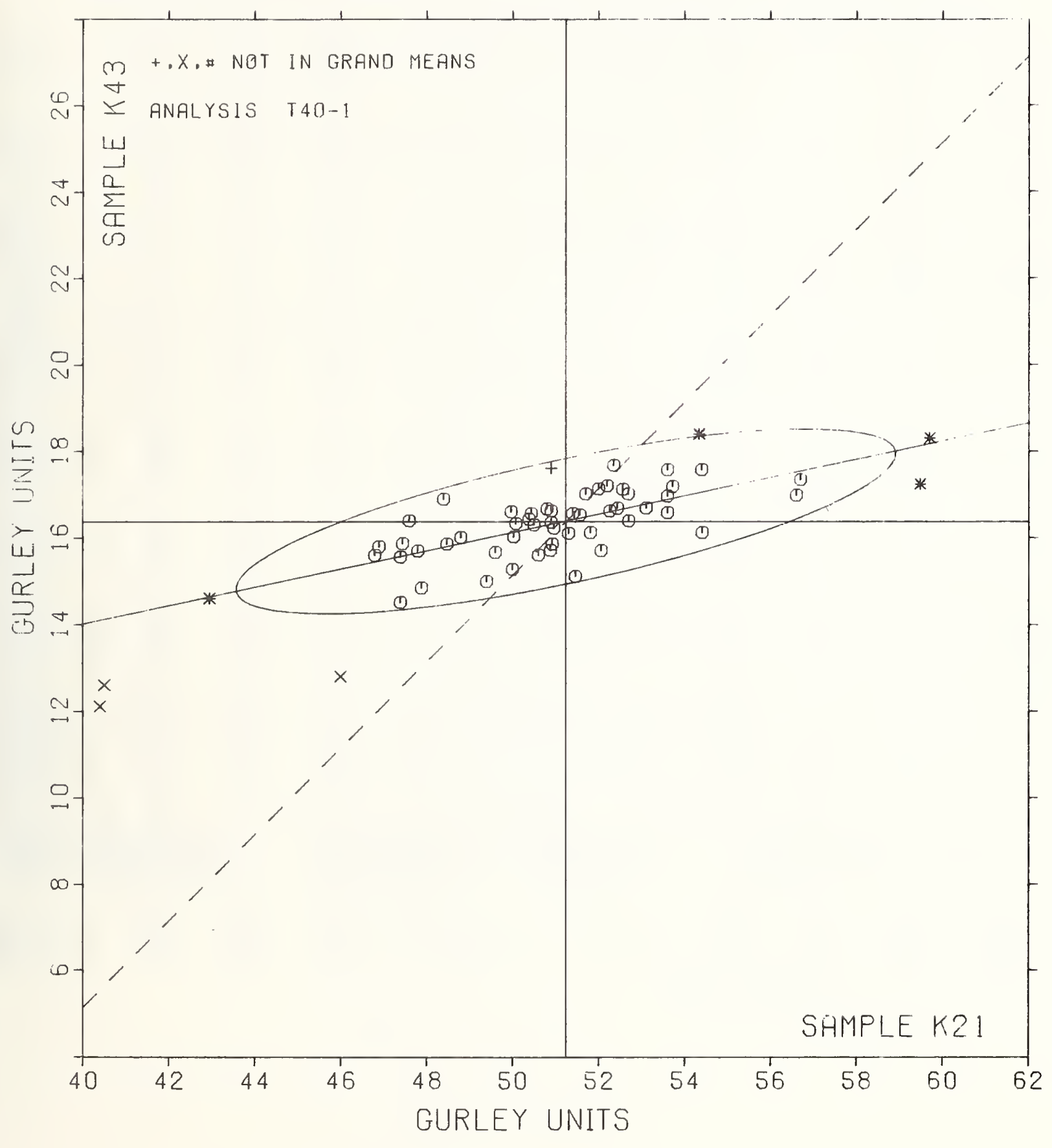



AIR RESISTANCE, SHEFFIELD UNITS (CC/MIN) FER 0.442 SQ. IN ( $3 / 4$ IN. DIA) ORIFIEE SHEFFIELD IESTBR IS STANDARD FER THIS ANALYSIS

\begin{tabular}{|c|c|c|c|c|c|c|c|c|c|c|c|c|c|}
\hline \multirow{3}{*}{$\begin{array}{l}\text { LAb } \\
C \in D E\end{array}$} & \multirow{3}{*}{$\begin{array}{c}\text { SAMPIE } \\
\text { R21 } \\
\text { MEAN }\end{array}$} & \multicolumn{4}{|c|}{ PRINIING } & \multirow{3}{*}{$\begin{array}{c}\text { SAMPLE } \\
\text { R } 43 \\
\text { MEAN }\end{array}$} & \multicolumn{4}{|c|}{ PLINTING } & \multirow[t]{2}{*}{ TEST } & $D_{\bullet}-$ & \multirow[t]{2}{*}{ - 10} \\
\hline & & 103 GFA: & $S$ PER S & SQUARE ME & VETER & & 60 GRA & S PER S & SQUARE ME & IETER & & & \\
\hline & & DEV & N.DEV & SDR & R. SDK & & DEV & $\mathrm{N} \bullet \mathrm{DEV}$ & SDR & $R_{\bullet}$ SDR & VAR & $\mathbf{F}$ & $\mathbf{L A B}$ \\
\hline 1114 & 79.4 & 8.3 & 1.40 & 4.9 & .82 & $177 \cdot 7$ & 10.1 & 1.10 & 13.0 & 1.48 & 405 & $\boldsymbol{\theta}$ & 1114 \\
\hline 121 & 89.0 & 17.9 & 3.03 & 5.8 & .97 & 172.9 & $5 \cdot 3$ & .58 & 5.8 & 1.11 & 405 & $*$ & 1121 \\
\hline L1245 & 70.2 & -.9 & .15 & 6.8 & 1.15 & 168.0 & .4 & .05 & 7.4 & .84 & 405 & $\boldsymbol{\theta}$ & $1124 \mathrm{~S}$ \\
\hline 1132 & 69.0 & -2.1 & .35 & 8.2 & 1.38 & 168.4 & .8 & .09 & 11.8 & 1.35 & 405 & 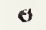 & 1132 \\
\hline 2148 & 79.9 & 8.8 & 1.49 & 4.5 & .75 & 174.6 & 7.0 & .77 & 8.9 & 1.02 & 405 & $\theta$ & 1148 \\
\hline L150 & 67.2 & -3.5 & -.066 & 4.4 & .74 & 194.5 & 26.9 & 2.93 & 23.9 & 2.72 & 405 & $*$ & L 150 \\
\hline L 157 & 67.8 & $-3 \cdot 3$ & -.56 & 5.8 & .97 & 174.6 & 7.0 & .77 & 10.9 & 1.25 & $40 \mathrm{~s}$ & A & 1157 \\
\hline L1 58 & 73.0 & 1.5 & . 32 & 5.9 & .99 & 162.0 & -5.6 &. .60 & 11.4 & 1.29 & $40 S$ & $\boldsymbol{\sigma}$ & I 158 \\
\hline $1173 B$ & 67.5 & -3.6 &. .61 & 4.9 & .82 & 177.0 & 9.4 & 1.03 & 4. 8 & .55 & 405 & $\boldsymbol{\theta}$ & L 1731 \\
\hline $\mathrm{L} 190 \mathrm{C}$ & 78.8 & 7.7 & 1.30 & 6.4 & 1.08 & 162.6 & -5.0 & -.54 & 8.8 & 1.00 & 405 & $\boldsymbol{\theta}$ & L $190 \mathrm{C}$ \\
\hline 1213 & 75.7 & 4.6 & .78 & 5.7 & .96 & 165.2 & -2.4 & -.26 & 8.1 & .92 & $40 \mathrm{~S}$ & $\theta$ & 1213 \\
\hline L223 & 62.1 & -9.0 & -1.52 & 3.7 & .62 & 163.0 & -4.6 & -.50 & 10.2 & 1.17 & 405 & $\theta$ & 1223 \\
\hline L228 & 06.4 & -4.7 &. .79 & 6.4 & 1.08 & 165.9 & -1.7 & .18 & 5.8 & .66 & 405 & 0 & L 228 \\
\hline $1230 \mathrm{~s}$ & 69.6 & -1.5 & .25 & 7.0 & 1.17 & 159.9 & -7.7 & .83 & 4.0 & .46 & 405 & 6 & L 230 S \\
\hline 1233 & 55.4 & -15.7 & -2.66 & 7.2 & 1.21 & 158.0 & -9.6 & -1.04 & 8.2 & .93 & $40 s$ & * & L 233 \\
\hline 1241 & 79.7 & 8.6 & 1.46 & $5 \cdot 7$ & .95 & 176.4 & 8.8 & .96 & 7.6 & .87 & 405 & $\boldsymbol{A}$ & 1241 \\
\hline 1249 & 65.9 & -5.2 & -.88 & 6.4 & 1.08 & 164.4 & -3.2 & -.34 & 10.0 & 1.14 & $40 \mathrm{~s}$ & $\boldsymbol{\theta}$ & 1249 \\
\hline L255 & 92.1 & 21.0 & 3.55 & 6.2 & 1.03 & 188.7 & $21 \cdot 1$ & 2.30 & 5.8 & .66 & 405 & $\mathbf{x}$ & L255 \\
\hline$L 257 \mathrm{~A}$ & 77.6 & 6.5 & 1.10 & 5.0 & .85 & 157.5 & -10.1 & $-1,09$ & 8.7 & 1.00 & 405 & $\boldsymbol{\theta}$ & L 2574 \\
\hline $1257 \mathrm{H}$ & 68.6 & -2.5 & -.42 & 5.6 & .94 & 169.0 & 1.4 & .16 & 13.2 & 1.51 & 405 & $\theta$ & 12578 \\
\hline $1257 \mathrm{C}$ & 78.5 & $7 \cdot 4$ & 1.25 & 8.0 & 1.35 & 161.7 & -5.9 & -.64 & 8.0 & .92 & 405 & $\boldsymbol{\theta}$ & L257C \\
\hline L260 & 71.2 & .1 & .02 & 6.7 & 1.12 & 173.4 & 5.8 & .64 & 13.9 & 1.59 & $4 \mathrm{Cs}$ & $\boldsymbol{\theta}$ & L 260 \\
\hline 12025 & 71.4 & - 3 & .05 & $4 \cdot 1$ & .68 & 157.2 & $=10.4$ & -1.13 & 8.4 & .96 & 405 & $\boldsymbol{\theta}$ & L 262S \\
\hline L288 & 73.3 & 2.2 & .37 & 4.1 & .69 & 187.3 & 19.7 & 2.15 & 13.6 & 1.55 & 405 & $\theta$ & L 288 \\
\hline 1301 & 68.2 & -2.5 &. .49 & 8. 3 & 1.40 & 168.6 & 1.0 & .11 & 9.4 & 1.07 & 405 & $\theta$ & 1301 \\
\hline L305 & 70.0 & -1.1 &. .19 & 5.8 & .97 & 171.0 & 3.4 & .37 & 6.5 & .74 & 405 & $\theta$ & L305 \\
\hline 2318 & 70.2 &. .5 & .015 & 6.4 & 1.07 & 158.5 & -9.1 & .98 & 5.6 & .64 & 405 & $\boldsymbol{\theta}$ & 2318 \\
\hline L352 & 78.5 & $7 \cdot 4$ & 1.25 & 2.3 & .39 & 168.6 & 1.0 & .11 & 8.7 & .99 & 405 & $A$ & L352 \\
\hline L354 & 73.6 & $2 \cdot 5$ & .42 & 6.8 & 1.15 & 168.8 & 1.2 & .14 & 9.3 & 1.06 & 405 & A & L354 \\
\hline L360 & 69.1 & -2.0 & -.34 & 2.8 & .48 & 157.2 & -10.4 & -1.13 & 6.5 & .74 & $40 \mathrm{~s}$ & $\boldsymbol{\theta}$ & L 360 \\
\hline 1370 & 67.3 & -3.8 & -.64 & 5.5 & .92 & 156.3 & -11.3 & -1.22 & 8.2 & .93 & 405 & A & L 370 \\
\hline 1372 & 69.5 & -1.6 & -.27 & 9.9 & 1.66 & 170.4 & 2.8 & .31 & 6.5 & .74 & 405 & $\boldsymbol{\sigma}$ & 1372 \\
\hline L350 & 66.5 & -4.6 & -.78 & 5.8 & .97 & 161.0 & -6.6 & -.71 & 8.4 & .96 & 405 & $\theta$ & L390 \\
\hline I562 & 71.5 & .4 & .07 & 6.3 & 1.06 & 181.8 & 14.2 & 1.55 & S.1 & 1.03 & 405 & $\boldsymbol{\theta}$ & 1562 \\
\hline L575 & 71.1 & .0 & .00 & 4.4 & .74 & 168.2 & .6 & .07 & 12.3 & 1.40 & $\triangle O S$ & ब & 1575 \\
\hline 1585 & 71.5 & .4 & .07 & 8.2 & 1.38 & 167.5 & -.1 & -.01 & 11.8 & 1.35 & 405 & $H$ & L5 85 \\
\hline L5؟7 & 70.6 & -.5 & -.08 & 6.5 & 1.16 & 158.9 & -8.7 & -.94 & 6.0 & .68 & 405 & 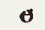 & 1597 \\
\hline 1600 & 71.0 & -.1 & -.02 & 7.1 & 1.20 & 160.2 & -7.4 & .80 & 5.5 & .63 & 405 & $\sigma$ & 1600 \\
\hline L626 & 64.4 & -6.7 & -1.13 & 8.8 & 1.47 & 146.3 & $-21 \cdot 3$ & -2.31 & 6.7 & .77 & 405 & H & L620 \\
\hline L684 & 64.5 & $-6 \cdot 6$ & -1.12 & 3.7 & .62 & 176.7 & 9.1 & .99 & 8.9 & 1.02 & 405 & $\boldsymbol{\theta}$ & 1684 \\
\hline 1687 & 69.1 & -2.0 & -.34 & 5.5 & . $\$ 2$ & 171.1 & 3.5 & .39 & 0.5 & .74 & 405 & $\boldsymbol{\theta}$ & 1687 \\
\hline GR. MEAN & 71.1 & SHEFF。 U & I IS & & GRAND MEAN - & $=167.6$ & SHEFF. $U$ & ITS & & TEST DETER & INATIF & NS & \\
\hline SD MEAHS & 5.5 & SHEFF。 UI & I Ts & & SD OF MEANS - & $=\quad 9.2$ & SHEFF. 0 & ITS & & 40 LABS I & GRAN & $\mathrm{m}$ & ANS \\
\hline & & AVER & GE SDR & 5.9 & SHEFF. UNITS & & AVER & GE SDR & 8.8 & SHEFF UN & & & \\
\hline L1 $82 \mathrm{~B}$ & $246 \cdot 5$ & 175.4 & 29.67 & 18.3 & 3.07 & 762.5 & 594.9 & 64.67 & 35.8 & 4.09 & $40 \mathrm{~B}$ & - & $1182 B$ \\
\hline L243日 & 280.2 & 209.1 & 35.37 & 24.5 & 4.12 & 821.4 & 653.8 & 71.07 & 52.2 & 5.95 & $4 \mathrm{CB}$ & - & L $243 B$ \\
\hline L312 & 80.0 & 8.5 & 1.51 & 3.8 & .63 & 167.5 & 01 & .01 & 6.5 & .74 & $\triangle O T$ & $\bullet$ & 1312 \\
\hline 1587 & 74.0 & 2.9 & .49 & 5.7 & .95 & 169.5 & 1.9 & .21 & 8.3 & .95 & $40 T$ & - & 1587 \\
\hline
\end{tabular}

TOTAL NUMBER GF LABGRATERIES FEPURTING - 45

Best values: $\mathrm{K} 2171+8$ Sheffield units

K43 167 \pm 14 Sheffield units 
AIR BESISIANCE, SHEFFIELD UNITS (CC/NIN) FGR $0.442 \mathrm{SQ}$. IN ( $3 / 4$ IN. DIA) ARIFICE SHEFFIELD TESTER IS STANDARD FAR THIS ANALYSIS

\begin{tabular}{|c|c|c|c|c|c|}
\hline \multicolumn{2}{|l|}{ LAB } & \multicolumn{2}{|c|}{ MEANS } & \multicolumn{2}{|c|}{ CEARDINATES } \\
\hline CADE & $F$ & B2 1 & B43 & MAJEE & MINER \\
\hline 2233 & $*$ & 55.4 & 158.0 & -12.6 & 13.3 \\
\hline L223 & $\boldsymbol{a}$ & 62.1 & 163.0 & -6.3 & 7.8 \\
\hline 1626 & $\boldsymbol{\theta}$ & $64 \cdot 4$ & 146.3 & $-22 \cdot 2$ & 2.1 \\
\hline 2684 & 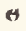 & $64 \cdot 5$ & 176.7 & $7 \cdot 6$ & 8.4 \\
\hline L249 & $\theta$ & 65.9 & $164 \cdot 4$ & $-4 \cdot 2$ & 4.4 \\
\hline L 228 & $\theta$ & 66.4 & 165.9 & $-2 \cdot 6$ & 4.2 \\
\hline L390 0 & $\theta$ & 66.5 & 161.0 & $-7 \cdot 4$ & 3.1 \\
\hline L150 & * & 67. & 194.5 & 25.5 & s. 5 \\
\hline L370 & $\theta$ & 67.3 & 156.3 & -11.8 & 1.3 \\
\hline $\mathrm{L} 173 \mathrm{~B}$ & $\theta$ & 67.5 & 177.0 & 8.5 & 5.5 \\
\hline L157 & a & 67.8 & 174.6 & 6.2 & 4.7 \\
\hline 1301 & a & 68.2 & 168.6 & .4 & 3.0 \\
\hline L25 7B & $\theta$ & 68.6 & 169.0 & .9 & 2.7 \\
\hline 1132 & $\theta$ & 69.0 & 168.4 & .4 & 2.2 \\
\hline 1687 & $\theta$ & 69.1 & 171.1 & 3.0 & 2.7 \\
\hline 1360 & $\theta$ & $6 S_{0} 1$ & 157.2 & -10.5 & -.2 \\
\hline L. 372 & $\theta$ & 69.5 & 170.4 & 2.4 & $2 \cdot 2$ \\
\hline $1230 \mathrm{~S}$ & A & 69.6 & 159.9 & -7.8 & -01 \\
\hline L305 & $\theta$ & 70.0 & 171.0 & 3.1 & 1.8 \\
\hline L124S & $\theta$ & 70.2 & 168.0 & .2 & 1.0 \\
\hline L. 318 & 9 & 70.2 & 158.5 & -9.0 & -1.0 \\
\hline L597 & $\theta$ & 70.6 & 158.9 & $-8 \cdot 6$ & $-1 \cdot 3$ \\
\hline 1600 & $\theta$ & 71.0 & 160.2 & -7.2 & -1.5 \\
\hline L575 & $\theta$ & 71.1 & 168.2 & .6 & .1 \\
\hline L260 & H & 71.2 & 173.4 & 5.7 & 1.1 \\
\hline L262S & $\theta$ & 71.4 & 157.2 & -10.1 & -2.5 \\
\hline L585 & $\theta$ & 71.5 & 167.5 & .0 & $=.4$ \\
\hline 1562 & e & 71.5 & 181.8 & 14.0 & 2.6 \\
\hline L158 & $\theta$ & 73.0 & 162.0 & -5.0 & -3.0 \\
\hline L2E 8 & $\theta$ & 73.3 & 187.3 & 19.8 & 2.0 \\
\hline L354 & $\theta$ & 73.6 & 168.8 & 1.7 & -2.2 \\
\hline L587 & - & 74.0 & 169.5 & 2.5 & -2.4 \\
\hline 1213 & $\theta$ & 75.7 & 165.2 & -1.3 & -5.0 \\
\hline L.257A & $\theta$ & 77.6 & 157.5 & -8.5 & -8.5 \\
\hline L 352 & $\theta$ & 78.5 & 168.6 & 2.6 & -7.0 \\
\hline $1257 \mathrm{C}$ & $\theta$ & 78.5 & 161.7 & -4.2 & -8.5 \\
\hline L190C & $\sigma$ & 78.8 & 162.6 & -3.2 & -8.6 \\
\hline 1114 & $\theta$ & 79.4 & 177.7 & 11.7 & -6.0 \\
\hline L24 I & $\theta$ & 79.7 & 176.4 & 10.5 & -6.6 \\
\hline L148 & $\theta$ & 75.9 & 174.6 & 8.7 & -7.1 \\
\hline $\mathrm{L} 312$ & - & 80.0 & 167.5 & 1.8 & -8.7 \\
\hline L121 & * & 89.0 & 172.9 & 9.0 & -16.4 \\
\hline L25 5 & $\mathrm{x}$ & 92.1 & 188.7 & 25.1 & -16.1 \\
\hline L182B & - & 246.5 & 762.5 & 618.5 & -46.6 \\
\hline L243B & - & 280.2 & 821.4 & 683.2 & -67.2 \\
\hline GN & & 71.1 & 167.6 & & \\
\hline & & & & & \\
\hline
\end{tabular}

AVG

R.SDR YAR PRUPERTY---TEST INSTRUMENT---CONDITIONS

$1.0740 S$ AIR RESISTANCE, SHEFFIELD (3/4 INCH DIAMETER ORIFICE) $8940 S$ AIR RESISTANCE, SHEFFIELD ( $3 / 4$ INCH DIAMETER ARIFICE) 1.1240 S AIR RESISTANCE, SHEFFIELD ( $3 / 4$ INCH DIAMETER ORIFICE) .8240 S AIR RESISTANCE, SHEFPIELD ( $3 / 4$ INCH DIAMETER GRIFICE) 1. $1140 S$ AIR RESISTANCE, SHEFFIELD ( $3 / 4$ INCH DIAMETER ORIFICE)

- 8740 S AIR RESISTANCE, SHEFFIELD ( $3 / 4$ INCH DIAMETER ARIFICE) $.9740 S$ AIR RESISTANCE, SHEFFIELD ( $3 / 4$ INCH DIAMETER ORIFICE) 1. 73 4OS AIR RESISTANCE, SHEFFIELD ( $3 / 4$ INCH DIAMETER ARIFICE) - $9240 S$ AIR RESISTANCE, SHEFFIELD ( $3 / 4$ INCH DIAMETER ORIFICE) $.6840 S$ AIR RESISTANCE, SHEFFIELD ( $3 / 4$ INCB DIAMETER GRIFICE)

$1.1140 S$ AIR RESISTANCE, SHEFPIELD (3/4 INCH DIAMETER GRIFICE) 1. 2340 S AIR RESISTANCE, SHEFFIELD ( $3 / 4$ INCH DIAMETER ORIFICE) 1. $2340 S$ AIR RESISTANCE, SHEFFIELD ( $3 / 4$ INCH DIANETER ARIFICE) 1. 37 4OS AIR RESISTANCE, SHEFFIELD (3/4 INCH DIAMETER ORIFICE) $.8340 S$ AIR RESISTANCE, SHEFFIELD ( $3 / 4$ INCH DIAMETER ORIFICE)

.61405 AIR RESISTANCE, SHEFFIELD ( $3 / 4$ INCH DIAMETER ORIFICE) 1.2040 S AIR RESISTANCE, SHEFFIELD (3/4 INCH DIAMETER ORIFICE) - 8240 S AIR RESISTANCE, SHEFFIELD ( $3 / 4$ INCH DIAMETER ARIFICE) - $8540 S$ AIR RESISTANCE, SHEFFIELD ( $3 / 4$ INCH DIAMETER ORIFICE) $1.0040 S$ AIR RESISTANCE, SHEFFIELD ( $3 / 4$ INCH DIAMETER ORIFICE)

- $8640 S$ AIR RESISTANCE, SHEFFIELD ( $3 / 4$ INCH DIAMETEE GRIFICE) - 9240 A AIR RES ISTANCE, SHEFFIELD (3/4 INCH DIAMETER ORIFICE)

- $9140 S$ AIR RESISTANCE, SHEFFIELD ( $3 / 4$ INCH DIAMETER GRIFICE) 1.07 40 S AI\& RESISTANCE, SHEFFIELD (3/4 INCH DIAMETER ORIFICE) 1. $3540 S$ AIR RESISTANCE, SHEFFIELD ( $3 / 4$ INCH DIAMEIER ORIFICE)

$.8240 S$ AIR RESISTANCE, SHEFFIELD ( $3 / 4$ INCH DIAMETER URIFICE) 1. 36 4OS AIR RESISTANCE, SHEFFIELD ( $3 / 4$ INCH DIAMETER ERIFICE) 1.04 40 S AIR RESISTANCE, SHEFFIELD ( $3 / 4$ INCH DIANETER ARIFICE) 1. $1440 S$ AIR RESISTANCE, SHEFFIELD ( $3 / 4$ INCH DIAMETER ERIFICE) 1.1240 S AIR RESISTANCE, SHEFFIELD ( $3 / 4$ INCH DIAMETER ORIFICE)

1. $1040 S$ AIR RESISTANCE, SHEFFIELD (3/4 INCH DIAMETER ORIFICE) - $9540 T$ AIR RESISTANCE, SHEFFIELD ( 3 INCH DIAMETEB GRIEICE)

- $9440 S$ AIR RESISTANCE, SHEFFIELD ( $3 / 4$ INCH DIAMETEF ORIFICE) - 92 4OS AIR RESISTANCE, SHEFFIELD (3/4 INCH DIAMETER GRIFICE) $.6940 S A I R$ RESISTANCE, SHEFFIELD (3/4 INCH DIAMETER ORIFICE)

$1.1340 S$ AIR RESISTANCE, SHEFFILLD ( $3 / 4$ INCH DIAMETER GRIFICE) $1.0440 S A I R$ RESISTANCE, SHEFFIELD (3/4 INCH DIAMETER GRIFICE) 1.15405 AIR RESISTANCE, SHEFFIELD ( $3 / 4$ INCH DIAMETER ARIFICE) - 91 $40 S$ AIR RESISTANCE, SHEFFIELD ( $3 / 4$ INCH DIAYETER ARIFICE) $.8840 S$ AIR RESISTANCE, SHEFFIELD (3/4 INCH DIAMETER GRIFICE)

-68 40 T AIR RESISTANCE, SHEFFIELD ( 3 INCB DIAMETER ORIFICE) 1. 04 4OS AIR RESISTANCE, SHEFFIELD (3/4 INCH DIAMETER ARIFICE) - 8540 S AIR RESISTANCE, SHEFFIELD (3/4 INCH DIAMETEE ARIFICE) $3.5840 B$ AIR RESISTANCE, BENDTSEN, MG 150 $5.0440 B$ AIR RESISTANCE, BENDTSEN, WG 150 1.00

ITH GAMMA $=77$ DEGREES 
AIR RESISTANCE, SHEFFIELD

SAMPLE K21 = 71. SHEFF. UNITS SAMPLE K43 $=168$. SHEFF. INHIIS

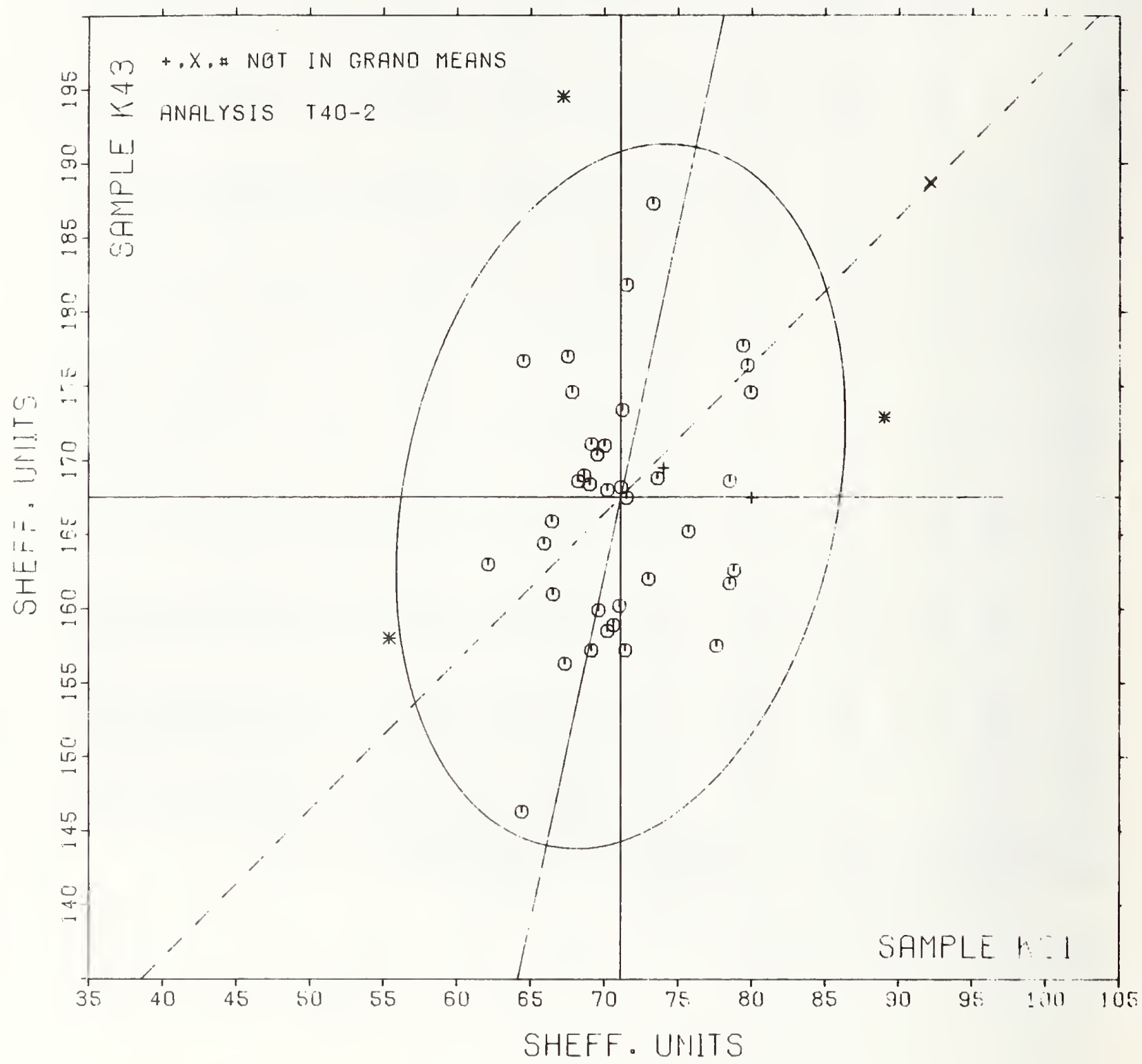


AIR RESISTANCE, HIGH RANGE, GURIEY MERCURY FLGTATIGN

DIRECT READING, SEC/10 CC, MERCURY DENSITY

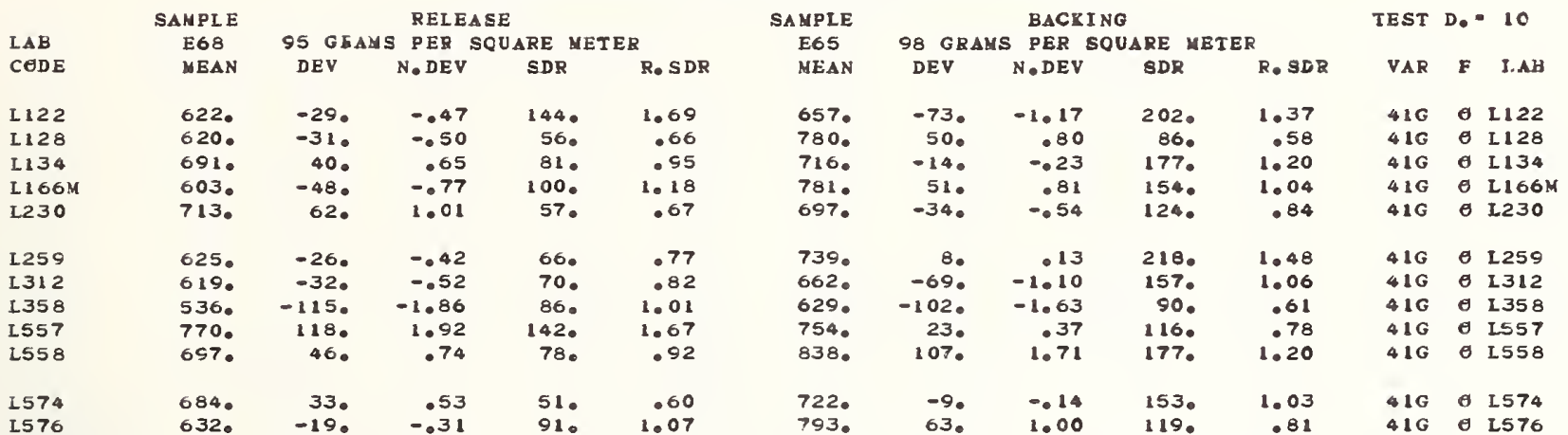

GR. MEAN = 651. SEC/10 CC GRAND MEAN - 731. SEC/10 CC

SD MEANS $=62$. SEC/10 CC AVERAGE SDR $=$ 85. SEC/10 CC

TETAL NUMBER GF LABERATERIES REPGRTING = 12

Best values: E68 650 seconds per 10cc,

E65 730 mercury density

(direct reading)

The values reported here are the time in
geconds required for the displacement of
10 mi of al through an area of lo o sq.
in of the gpecimen. The values are not
converted to looml of afr nor to oil density.
REPERT NO. 586

\begin{tabular}{|c|c|c|c|c|c|c|c|c|c|c|c|c|c|c|}
\hline \multirow{3}{*}{$\begin{array}{l}\text { LAB } \\
\text { CEDE } \\
\text { L358 }\end{array}$} & \multirow{3}{*}{$\begin{array}{l}\boldsymbol{F} \\
\boldsymbol{\theta}\end{array}$} & \multicolumn{2}{|c|}{ MEANS } & \multicolumn{2}{|c|}{ COURDINATES } & \multirow{2}{*}{$\begin{array}{l}\text { AVG } \\
R_{\bullet} \text { SDR }\end{array}$} & \multirow[b]{2}{*}{ VAR } & \multirow{2}{*}{\multicolumn{2}{|c|}{ PREPERTY $=--$ TEST }} & \multirow{2}{*}{\multicolumn{4}{|c|}{ T INSTRUMENT $-\infty$ CENDITIENS }} & \multirow[b]{3}{*}{ FLETATIEN } \\
\hline & & E68 & E65 & MAJEF & MINER & & & & & & & & & \\
\hline & & 536. & 629. & -153 & 12 & $\cdot 81$ & 416 & $\Delta I R$ & RES ISTANCE, & HIG H & RANGE, & GURLEY & MERCURY & \\
\hline L $166 \mathrm{M}$ & $\theta$ & 603. & 781. & 3. & 70. & 1.11 & $41 \mathrm{G}$ & AI $\mathbf{K}$ & RESISTANCE. & HIG H & RANGE, & GURLEY & MERCURT & FLETATIEN \\
\hline 1312 & $\boldsymbol{\theta}$ & 619. & 662. & -72. & -25 & .94 & $41 \mathrm{G}$ & $\mathbf{A I} \mathbf{R}$ & RESISTANCE. & HI G H & RANGE. & GURLEY & MERCURY & FLETATIEN \\
\hline L 128 & $\theta$ & 620. & 780. & 14. & 57. & .62 & $41 \mathrm{G}$ & AI R & RESISTANCE, & HI GH & RANGE, & G TRLEY & MERCORY & FLETATIEN \\
\hline L122 & G & 622. & 657 & -73 & -30 & 1.53 & $41 G$ & AIR & RESISTANCE, & НI G В & RANGE。 & GDRLEY & MERCORY & FLETATIEN \\
\hline L259 & $\boldsymbol{\theta}$ & 625. & 739. & -13 & 24. & 1.13 & $41 \mathrm{G}$ & AI R & RESISTANCE, & HI GH & RANGE, & GURLEY & MERCORY & FLETATIEN \\
\hline 2576 & $\boldsymbol{\theta}$ & 632 & 793. & 32. & 57. & .94 & $41 \mathrm{G}$ & $\mathbf{A I} \mathbf{R}$ & RESISTANCE, & HIG H & RANGE, & GORLEY & MERCURY & FLETATIEN \\
\hline 2574 & 9 & 684. & 722 & 17. & -30 & .82 & $41 \mathrm{G}$ & AIR & RESISTANCE, & HIG B & RANGE, & GURLEY & MBRCURY & FLETATIEN \\
\hline L 134 & ต & 691. & 716. & 18. & -39. & 1.08 & $41 G$ & $\mathbf{A I R}$ & RESISTANCE, & $\mathrm{HIGH}$ & RANGE, & GURLEY & MERCURY & FLETATIEN \\
\hline L558 & $\boldsymbol{\theta}$ & 697. & 838 & 109. & 42. & 1.06 & $41 G$ & $\Delta I R$ & RESISTANCE, & HIG H & RANGE, & GURLEY & MERCURY & FLETATIEN \\
\hline L230 & $\theta$ & 713. & 697. & 19. & -68 & .75 & $41 \mathrm{G}$ & AI R & RESISTANCE, & HI G H & RANGE, & GURLEY & MERCURY & FLETATIGN \\
\hline 1557 & $\theta$ & 770. & 754. & 99. & -69 & 1.22 & $41 \mathrm{G}$ & AIR & RES IST ANCE, & HIG B & RANGE, & GURLEY & MERCURY & FLETATION \\
\hline GXEANS & & $\begin{array}{l}651 . \\
95 \%\end{array}$ & $\begin{array}{r}731 \\
\text { ELIIPSE: }\end{array}$ & 218 & 149. & $\begin{array}{l}1.00 \\
\text { WITH }\end{array}$ & A $\mathbf{A}$ & A & 45 DEGREES & & & & & \\
\hline
\end{tabular}


AIR RESISTANCE, GURLEY HG FLOTATIGN

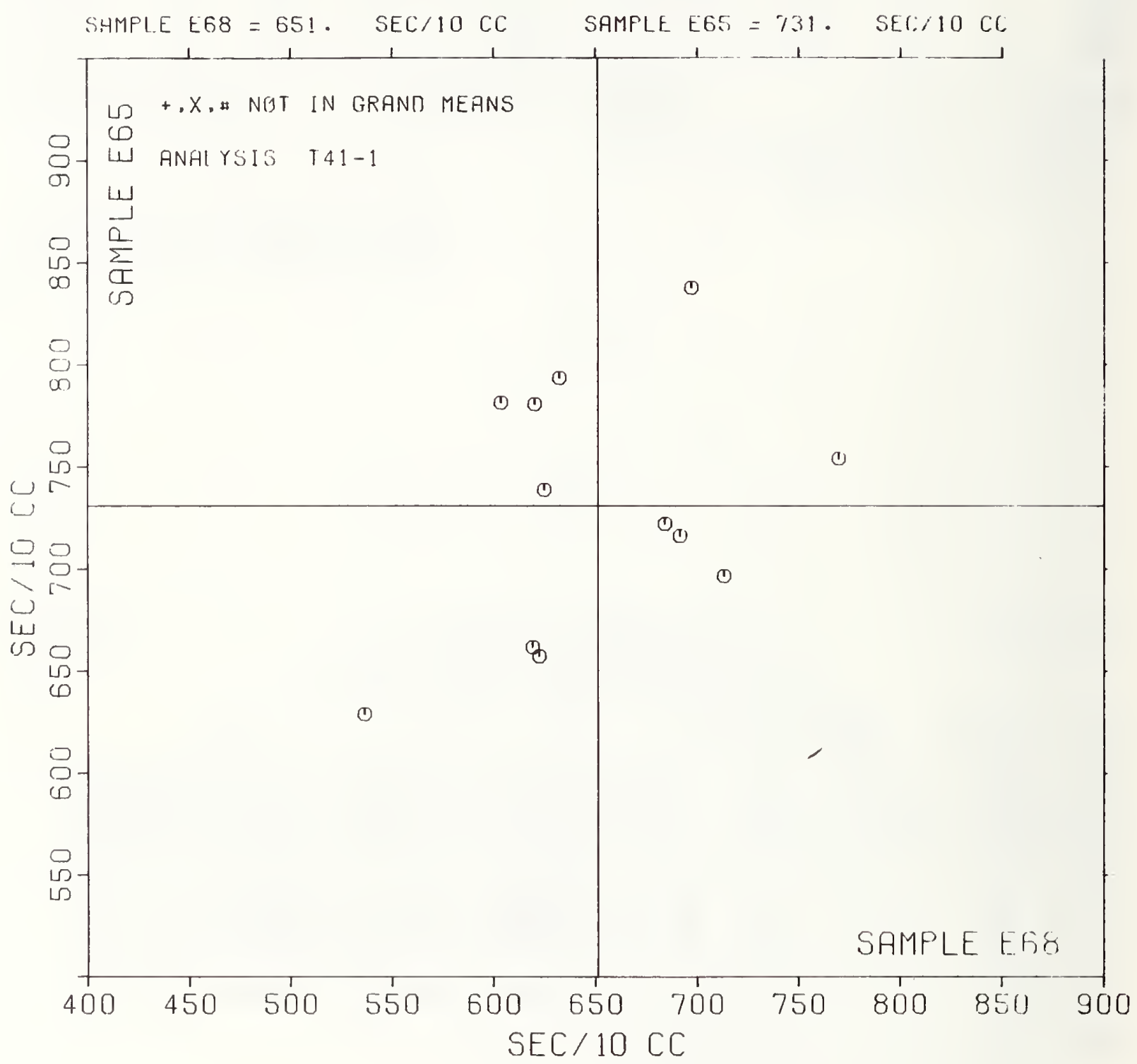




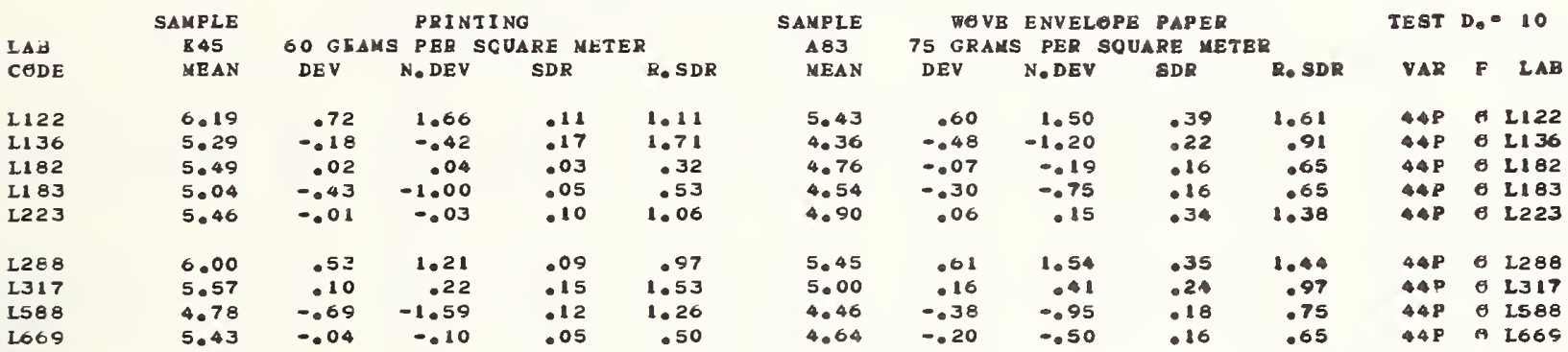

GR. MEAN $=5.47$ MICRENS GRAND MEAN $=4.84$ MICRANS SD MEANS - 43 MICKENS

TCTAL NUMBER GF IABCFATORIES KEPORTING - 9

Best values: $\mathrm{K} 45 \quad 5.5$ microns

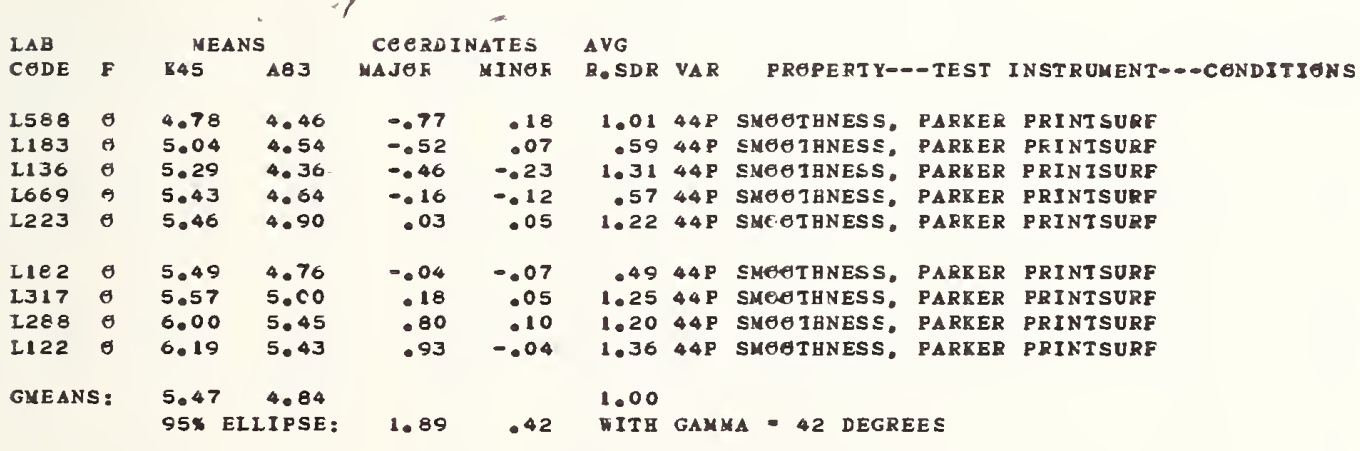


SHEFPIELD IESTER IS STANDAED FGR THIS ANALYSIS

\begin{tabular}{|c|c|c|c|c|c|c|c|c|c|c|c|c|c|}
\hline \multirow{3}{*}{$\begin{array}{l}\text { LAB } \\
\text { CADE }\end{array}$} & \multirow{3}{*}{$\begin{array}{c}\text { SAMPLE } \\
\text { K } 45 \\
\text { MEAN }\end{array}$} & \multicolumn{4}{|c|}{ PRINIING } & \multirow{3}{*}{$\begin{array}{c}\text { SAMPLE } \\
\text { AB3 } \\
\text { MEAN }\end{array}$} & \multicolumn{4}{|c|}{ WAVE ENVELUPE PAPER } & \multirow[t]{2}{*}{ TEST } & \multirow[t]{2}{*}{$D_{0}=$} & \multirow[t]{2}{*}{$=15$} \\
\hline & & $60 \mathrm{GFAl}$ & $S$ PER S & SQUARE $\triangle B$ & & & 75 GRA & $S$ PER S & SQUARE ME & & & & \\
\hline & & DEV & N.DEV & SDR & R. SDR & & DEV & $\mathrm{N} \bullet \mathrm{DEV}$ & SDR & $R \cdot S D R$ & VAR & $\boldsymbol{F}$ & $\mathbf{L A B}$ \\
\hline 2100 & 162.5 & $2 \cdot 3$ & .32 & 5.0 & .55 & 96.2 & -9.2 & -1.36 & 6.9 & .65 & 455 & $\theta$ & 2100 \\
\hline 2108 & 155.0 & -5.3 &. .75 & 6.2 & .68 & 106.7 & 1.3 & .19 & 8.7 & .81 & 455 & o & 2108 \\
\hline L 114 & $164 \cdot 1$ & $3 \cdot 8$ & .54 & 9.9 & 1.08 & 98.6 & -6.8 & -1.00 & 11.4 & 1.06 & $45 \mathrm{~S}$ & ต & 2114 \\
\hline L121 & $156 \cdot 3$ & -4.0 & -.57 & 7.4 & .80 & 106.1 & .7 & .10 & 11.5 & 1.08 & 455 & $\sigma$ & 2121 \\
\hline L 122 & 161.9 & 1.6 & .23 & 8.4 & .92 & 112.1 & 6.7 & .98 & 13.3 & 1.24 & 455 & $\theta$ & L1 22 \\
\hline L 123 & $1 \in 3.4$ & 3.1 & .45 & 11.2 & 1.23 & 101.9 & -3.6 & -.52 & 11.4 & 1.06 & 455 & $\sigma$ & L123 \\
\hline L124 & 153.6 & -6.7 & -.95 & 11.0 & 1.20 & 103.1 & -2.4 & -.35 & 11.0 & 1.03 & $45 S$ & $\theta$ & 1124 \\
\hline 1125 & 167.0 & 6.7 & .96 & 11.5 & 1.25 & 103.0 & -2.4 &. .36 & 16.8 & 1.56 & 455 & $\theta$ & L125 \\
\hline L1 26 & $164 \cdot 5$ & 4.3 & .61 & 15.3 & 1.68 & $106 \cdot 2$ & .8 & .11 & 14.8 & 1.38 & $45 \mathrm{~S}$ & 0 & L126 \\
\hline 1128 & 160.5 & .2 & .03 & 11.7 & 1.28 & 102.9 & -2.6 & -.38 & 8.7 & .82 & $45 s$ & $\boldsymbol{\theta}$ & L128 \\
\hline L132 & 169.5 & $9 \cdot 3$ & $1 \cdot 32$ & 13.6 & 1.48 & 101.3 & -4.2 &. .61 & 14.3 & 1.33 & 455 & $\boldsymbol{\sigma}$ & 1132 \\
\hline L134 & 150.7 & -9.6 & -1.37 & 7.5 & .82 & 97.7 & -7.8 & -1.14 & 6.2 & .58 & $45 S$ & 6 & $L_{1} 34$ \\
\hline L139s & 163.9 & 3.6 & .51 & 6.7 & .74 & 118.5 & 13.1 & 1.92 & 9.0 & .84 & 455 & $\boldsymbol{\sigma}$ & L139S \\
\hline 2148 & 169.0 & 8.7 & 1.24 & 9.5 & 1.03 & 116.3 & 10.8 & 1.59 & 13.2 & 1. 23 & 455 & $\boldsymbol{\theta}$ & L1 48 \\
\hline L150 & 170.9 & 10.6 & 1.51 & 18.6 & 2.03 & 105.5 & .0 & .00 & 14.9 & 1.39 & 455 & 0 & L150 \\
\hline L152 & $1 \in 3 \cdot 2$ & 2.9 & .42 & 5.4 & .59 & 111.7 & 6.3 & .92 & 11.3 & 1.06 & 455 & $\theta$ & L152 \\
\hline 1153 & 173.7 & 13.4 & 1.91 & 7.3 & .79 & 114.7 & 9.2 & 1.35 & 7.6 & .71 & $45 \mathrm{~S}$ & $\theta$ & 2153 \\
\hline 1157 & $168 \cdot 5$ & 8. 2 & 1.17 & 11.5 & 1.26 & 116.6 & 11.2 & 1.64 & 14.5 & 1.36 & 455 & $\theta$ & L157 \\
\hline L158 & 158.0 & -2.3 & -.32 & 7.3 & .79 & 110.7 & 5.2 & .77 & 10.2 & .95 & $45 \mathrm{~S}$ & $\boldsymbol{\theta}$ & 2158 \\
\hline 1159 & 170.1 & 9.8 & 1.39 & 11.8 & 1.28 & 114.9 & 9.5 & 1.39 & 10.7 & 1.00 & $45 \mathrm{~s}$ & $\sigma$ & L159 \\
\hline L 162 & 156.7 & -3.6 &. .51 & 6.2 & .67 & 107.0 & 1.6 & - 23 & 7.0 & .65 & 455 & e & L162 \\
\hline L166 & 153.9 & $-6 \cdot 3$ & -.90 & 8.0 & .87 & $109 \cdot 3$ & 3.9 & .57 & 9.2 & .86 & $45 S$ & $\boldsymbol{\theta}$ & L166 \\
\hline L167 & 162.3 & $2 \cdot 1$ & .29 & 4.2 & .46 & 112.7 & $7 \cdot 2$ & 1.06 & 5.0 & .46 & $45 \mathrm{~S}$ & $\boldsymbol{A}$ & L 167 \\
\hline $1173 B$ & 161.7 & 1.4 & .20 & $7 \cdot 5$ & .82 & 88.7 & -16.8 & -2.46 & 6.9 & .65 & $45 \mathrm{~S}$ & $*$ & $L_{1}>3 B$ \\
\hline L1835 & 162.9 & 2.7 & .38 & 8.8 & .96 & 111.9 & 6.4 & .94 & 16.7 & 1.56 & 455 & $\theta$ & 21835 \\
\hline L $190 \mathrm{C}$ & 159.4 & $\because 9$ & .012 & 7.4 & .81 & $108 \cdot 3$ & 2.9 & .42 & 9.2 & .86 & 455 & $\theta$ & L190C \\
\hline L190R & 157.1 & -3.1 & -.45 & 9.3 & 1.01 & 93.1 & $-12 \cdot 3$ & -1.81 & 12.0 & 1.11 & 455 & a & LI GOR \\
\hline 1206 & 163.6 & 3.3 & .47 & 6.3 & .68 & 114.4 & 9.0 & 1.31 & 11.6 & 1.08 & 455 & $\boldsymbol{\theta}$ & L206 \\
\hline L2 11 & $149 \cdot 4$ & -10.9 & -1.55 & 7.4 & .81 & 101.1 & -4.4 & -.64 & 10.5 & .98 & $45 \mathrm{~S}$ & $\boldsymbol{\theta}$ & 1211 \\
\hline L213 & 146.3 & -13.9 & -1.99 & 10.4 & 1.14 & 103.2 & $-2 \cdot 2$ & -.33 & 10.2 & .95 & $45 \mathrm{~s}$ & $\theta$ & 1213 \\
\hline 1219 & $170 \cdot 2$ & 9.5 & 1.41 & 16.9 & 1.85 & $108 \cdot 3$ & 2.9 & .42 & 10.8 & 1.01 & $45 s$ & 0 & 1219 \\
\hline L223 & 157.7 & -2.6 & -.37 & 6,3 & .69 & 103.9 & -1.5 & -.22 & 14.0 & 1.31 & $45 \mathrm{~S}$ & $\theta$ & L2 23 \\
\hline $1226 B$ & 149.9 & -10.3 & -1.47 & 9.1 & 1.00 & 96.1 & -9.3 & $-1 \cdot 37$ & 11.2 & 1.05 & 455 & $\boldsymbol{\theta}$ & L226B \\
\hline L228 & 163.3 & 3. 1 & .44 & 10.5 & 1.15 & 108.1 & 2.7 & .39 & 13.4 & 1.25 & $45 s$ & $\theta$ & L228 \\
\hline L230s & 162.1 & 1.5 & .26 & 8.6 & .94 & 101.5 & -3.9 &. .57 & 10.6 & .99 & $45 s$ & $\theta$ & L230S \\
\hline L231 & 167.1 & 6.8 & .97 & 9.5 & 1.04 & 103.8 & -1.6 & -.24 & 13.0 & 1.21 & $45 s$ & $\theta$ & L231 \\
\hline L232S & 160.3 & .1 & .01 & 5.8 & .64 & 92.7 & -12.8 & -1.87 & 8.8 & .82 & 455 & $\theta$ & L232S \\
\hline L233 & 155.2 & -5.1 &. .72 & 11.6 & 1.26 & 105.6 & .2 & .02 & 10.6 & .99 & $45 \mathrm{~S}$ & $\theta$ & L233 \\
\hline L237 & 160.8 & .5 & .07 & 5.2 & .56 & 106.0 & .6 & .08 & $4 \cdot 3$ & .40 & 455 & $\theta$ & L237 \\
\hline L241 & 150.1 & -10.1 & -1.44 & 8.4 & .92 & s8.9 & -6.6 & .096 & 7.7 & .71 & $45 \mathrm{~s}$ & $\boldsymbol{\sigma}$ & L 241 \\
\hline 1249 & 156.1 & $-4 \cdot 1$ & -.59 & 8.3 & .90 & 106.1 & .6 & .09 & 10.3 & .96 & $45 s$ & $\theta$ & L249 \\
\hline 1254 & $166 \cdot 7$ & 6.4 & .91 & 10.3 & 1.12 & 106.5 & 1.1 & .16 & 8.4 & .78 & $45 s$ & $\boldsymbol{\theta}$ & L254 \\
\hline L25 5 & $161 \cdot 3$ & 1.0 & .14 & 4.3 & .47 & 120.6 & 15.2 & 2.22 & 2.6 & .24 & 455 & $\theta$ & I255 \\
\hline L257A & $157 \cdot 1$ & -3.1 & -.45 & 10.2 & 1.11 & 102.8 & -2.6 & -.39 & 7.7 & .72 & 455 & $\boldsymbol{\theta}$ & I257A \\
\hline L257B & 165.3 & 5.1 & .72 & 9.9 & 1.08 & 103.2 & -2.2 & -.33 & 9.7 & .90 & 455 & $\theta$ & L257B \\
\hline L257C & 160.0 & -.3 & -.04 & 7.1 & .78 & 106.5 & 1.1 & .16 & 7.7 & .72 & $45 \mathrm{~S}$ & 9 & L257C \\
\hline L259 & 174.2 & 13.5 & 1.98 & 14.8 & 1.62 & 116.4 & 11.0 & 1.61 & 10.4 & .97 & 455 & $\boldsymbol{\theta}$ & L259 \\
\hline L260 & 160.6 & $\cdot 3$ & .05 & 6.2 & .67 & 111.4 & 6.0 & • 87 & 16.2 & 1.51 & $45 S$ & $\theta$ & L260 \\
\hline L20 1 & 156.4 & -3.9 & -.55 & $7 \cdot 3$ & .80 & $103 \cdot 3$ & -2.2 & -.32 & 8.6 & .80 & $45 S$ & $\boldsymbol{\theta}$ & L261 \\
\hline L262 & $157 \cdot 6$ & -2.7 & -.38 & $8 \cdot 3$ & .91 & 102.8 & -2.6 & -.39 & 8.3 & .78 & 455 & $\theta$ & L262 \\
\hline L275 & 156.7 & -3.5 & -.50 & 12.2 & 1.33 & 102.9 & -2.6 & -.38 & $13 \cdot 7$ & 1.28 & $45 S$ & $\boldsymbol{\theta}$ & L275 \\
\hline 1278 & 167.0 & 6.7 & .96 & 8.9 & .97 & 113.7 & 8.2 & 1.21 & 13.3 & 1.24 & $45 S$ & 6 & 1278 \\
\hline L28 1 & 162.7 & 2.4 & .34 & 10.2 & 1.11 & 109.9 & 4.5 & .66 & 14.5 & 1.35 & $45 s$ & $\theta$ & L281 \\
\hline L285 & 148.7 & -11.6 & -1.65 & 8.3 & .91 & 101.7 & -3.8 & -.55 & 12.5 & 1.16 & $45 S$ & $\theta$ & 1285 \\
\hline L28 8 & $169 \cdot 2$ & . $\quad 8.9$ & $1 \cdot 27$ & 7.7 & -84 & 106.9 & 1.4 & .21 & 11.2 & 1.04 & 455 & $\theta$ & L 288 \\
\hline L290 & 152.3 & -7.5 & -1.13 & 7.3 & .80 & 98.3 & -7.1 & -1.04 & 7.5 & .70 & $45 s$ & $\theta$ & L290 \\
\hline L29 is & 162.4 & 2.1 & .30 & 5.8 & .63 & 103.9 & -1.6 & -.23 & 11.2 & 1.04 & 455 & $\boldsymbol{\theta}$ & L291s \\
\hline 1301 & 163.0 & 2.7 & .39 & 7.4 & .81 & 116.3 & 10.8 & 1.59 & 12.4 & 1.15 & 455 & $\theta$ & L301 \\
\hline L305 & $165 \cdot 2$ & 4.9 & .70 & 8.4 & .92 & 109.1 & 3.6 & .53 & 7.4 & .69 & 455 & $\theta$ & 1305 \\
\hline L308 & 156.9 & -3.4 &. .49 & 7.0 & .77 & $\$ 9.5$ & -6.0 & $-0 \theta$ & 11.2 & 1.05 & $45 \mathrm{~s}$ & $\theta$ & 2308 \\
\hline 1312 & 161.4 & 1.1 & .16 & 13.4 & 1.46 & 115.0 & 9.6 & 1.40 & 9.4 & $.8 \theta$ & 455 & $\boldsymbol{\sigma}$ & L312 \\
\hline 2,317 & 157.3 & -3.0 & -.43 & 13.4 & 1.46 & 54.1 & -11.4 & -1.67 & 7.5 & .70 & $45 \mathrm{~s}$ & 0 & 2317 \\
\hline 2318 & 160.9 & .6 & .08 & 11.0 & 1.20 & 117.5 & 12.1 & 1.77 & 16.7 & 1.55 & $45 s$ & $\boldsymbol{A}$ & L31B \\
\hline L3 21 & 140.8 & -19.5 & -2.77 & 5.6 & .61 & 98.7 & -6.7 & -.98 & 12.4 & 1.15 & 455 & $\bullet$ & 1321 \\
\hline L 323 & 164.0 & 3.7 & .53 & 10.4 & 1.13 & 103.7 & -1.8 & .026 & 9.5 & .89 & 455 & a & L 323 \\
\hline
\end{tabular}


SHEFFIELD TESTER IO STANDARD FER THIS ANALYSIS

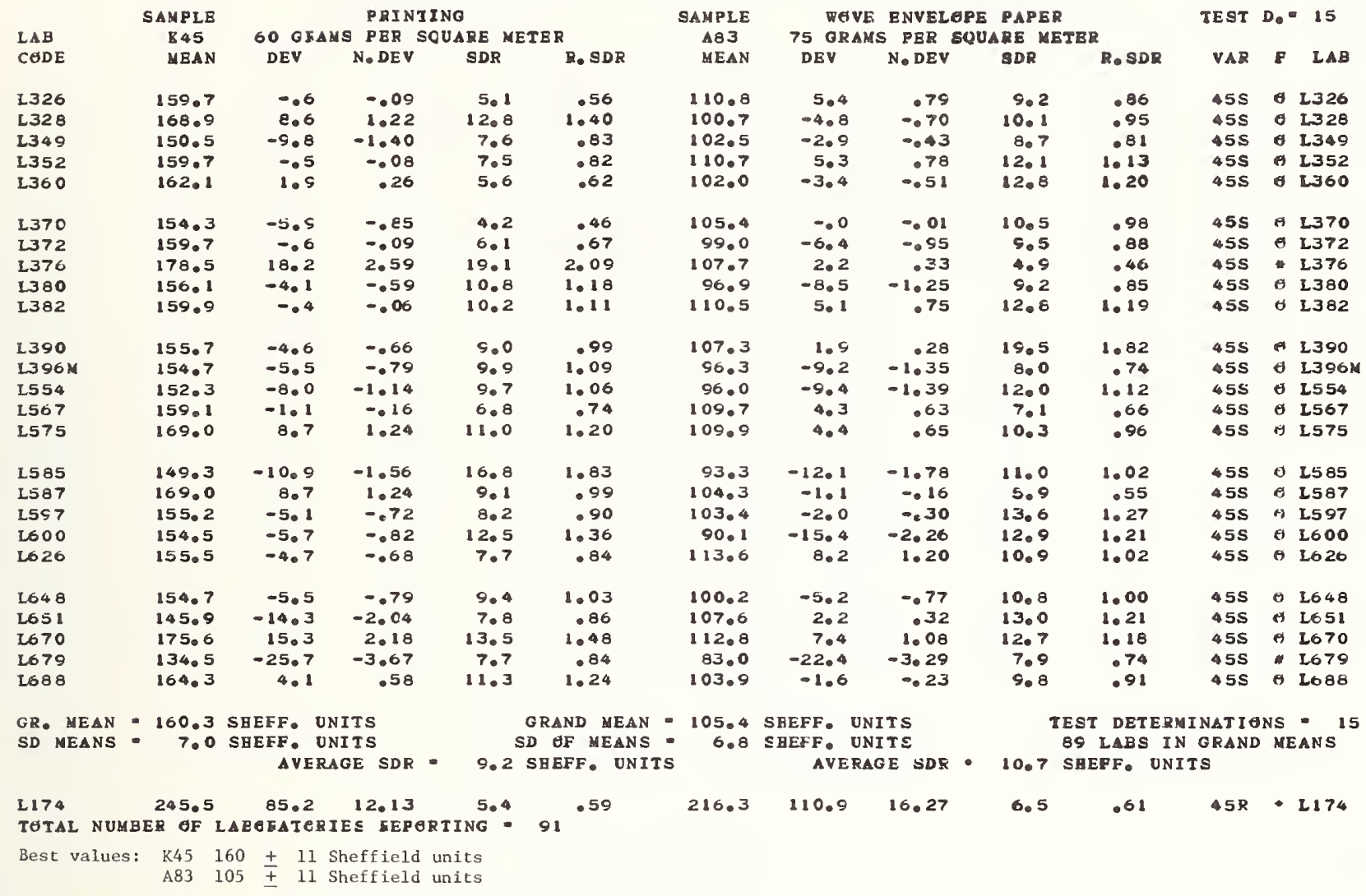

The following laboratories were omitted from the

grand means because of extreme test results: 679 . 


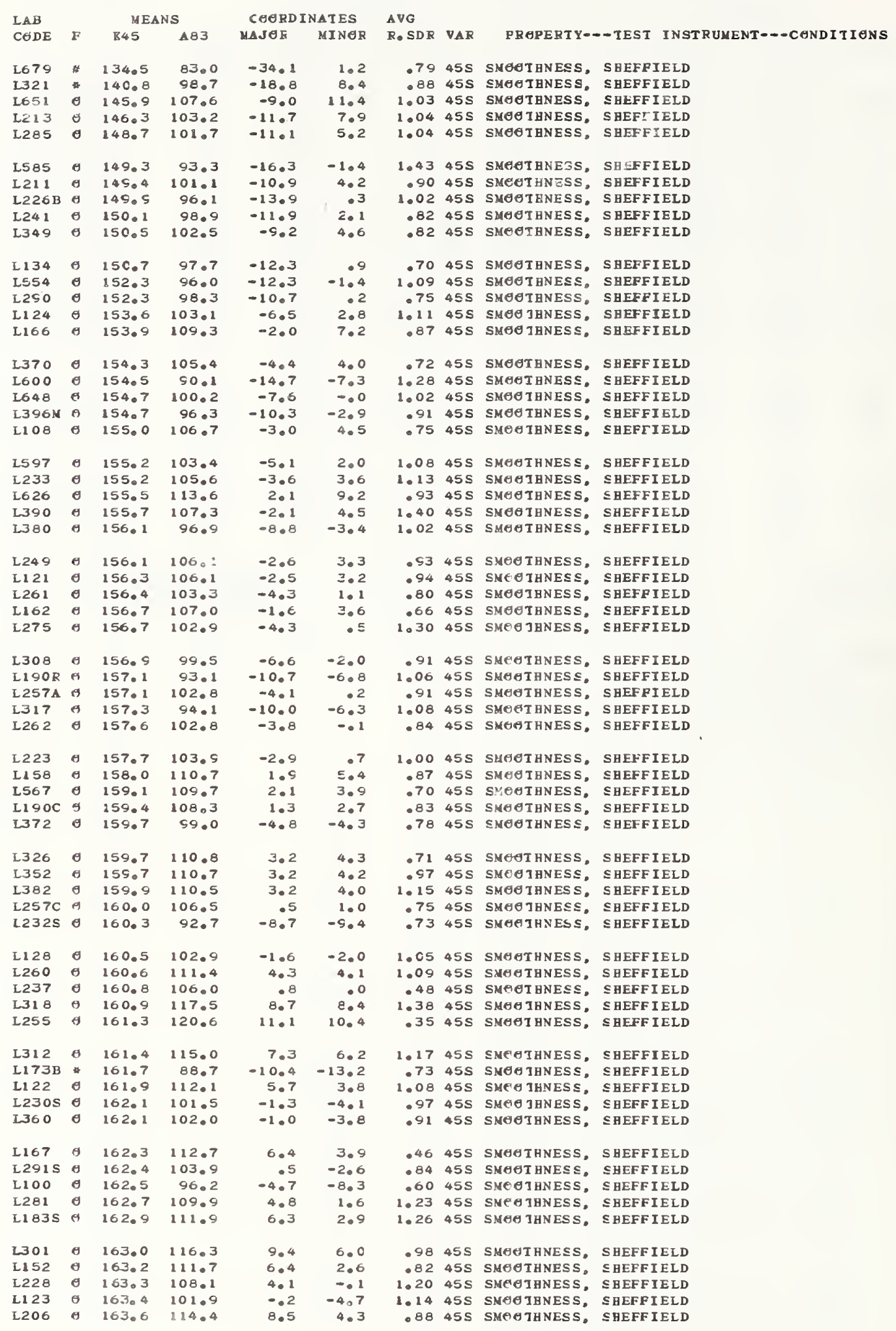


SHEFFIELD TESTER IS STANDARD FOR TEIS ANALYSIS

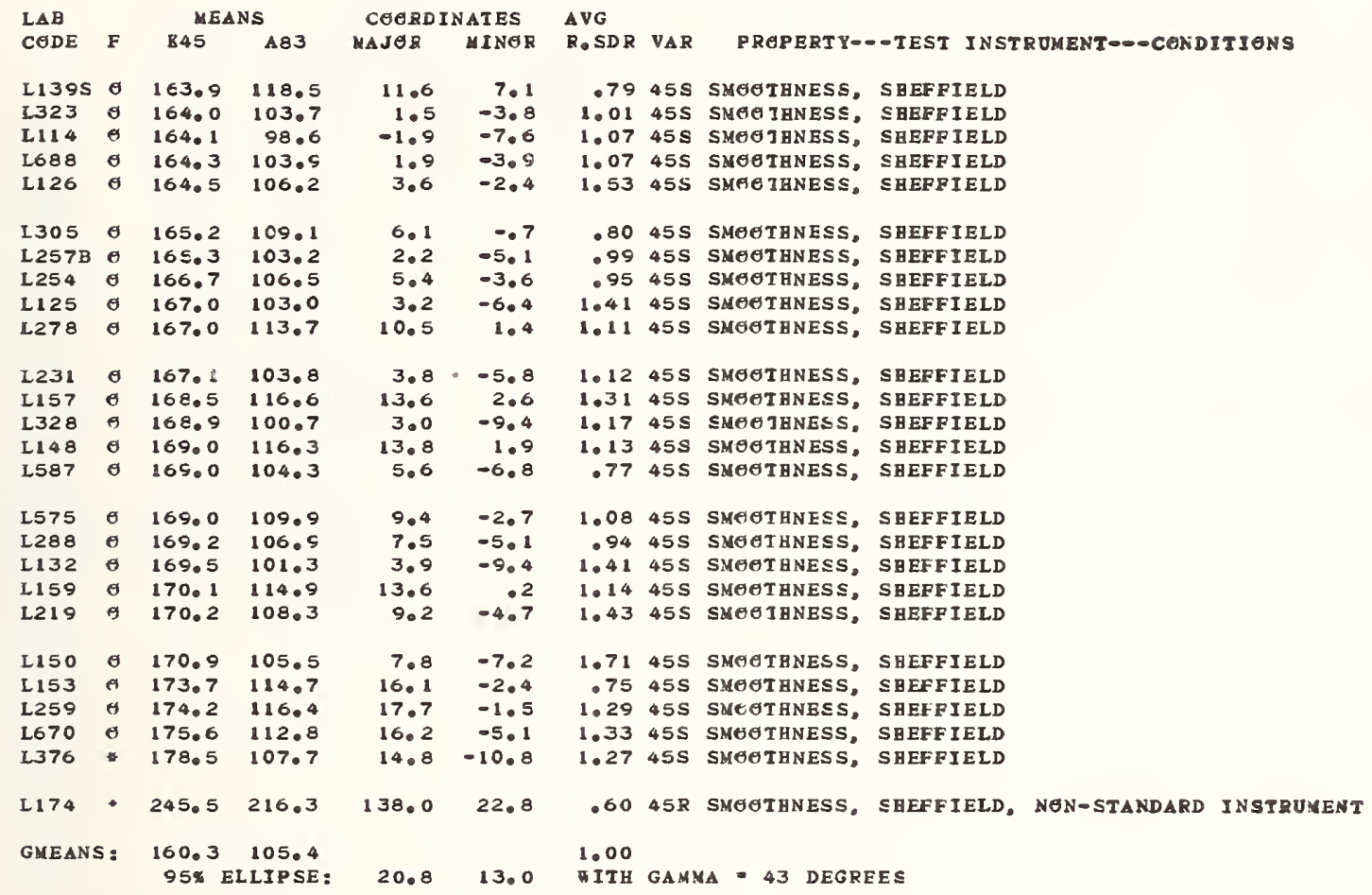


SMOOTHNESS, SHEFFIELD

SAMPLE K45 $=160$. SHEFF. UNITS SAMPLE A83 $=105$. SHEFF. UNITS

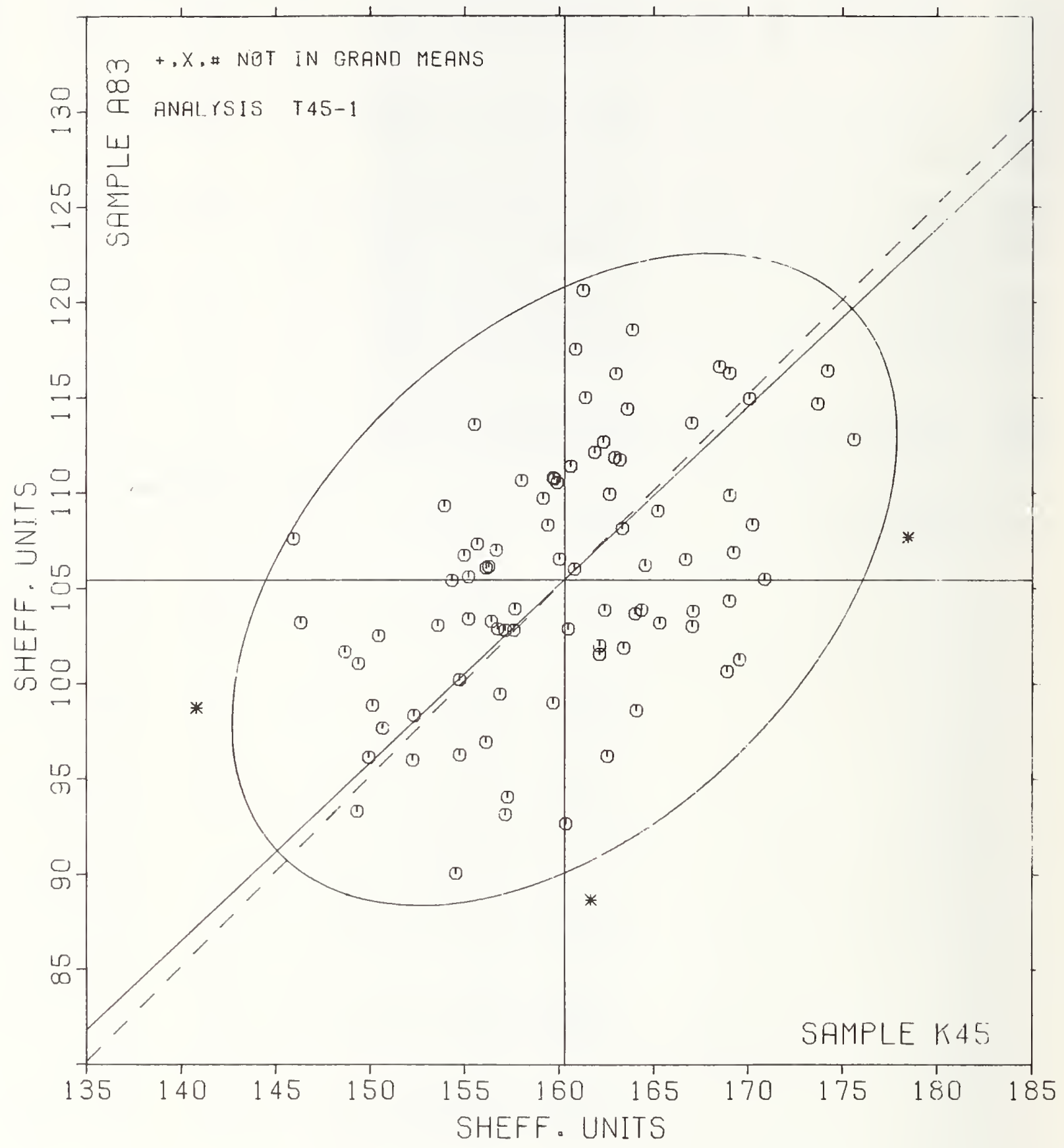


SMUOIHNESS, BEXK SECONDS

TAPPI SUGGESTED METHOD T479 SU-71, SNOOTHNESS EF PAPER (BEKE METHED)

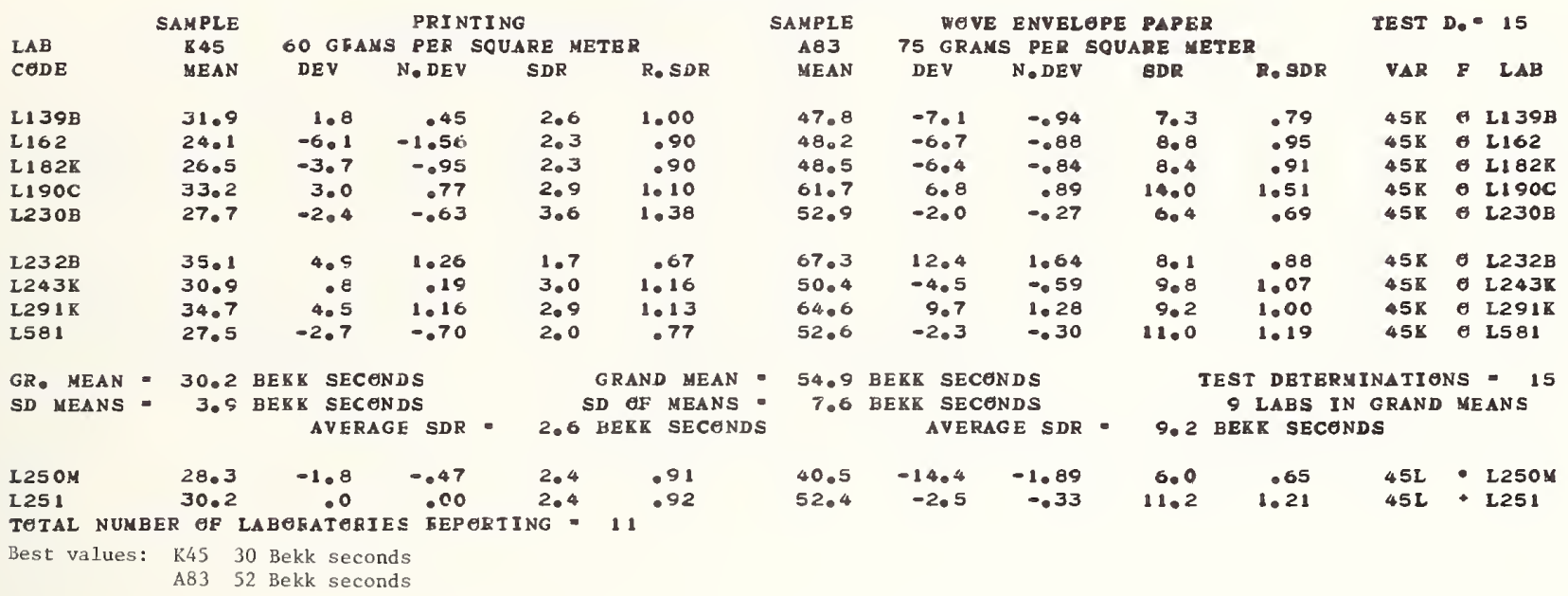

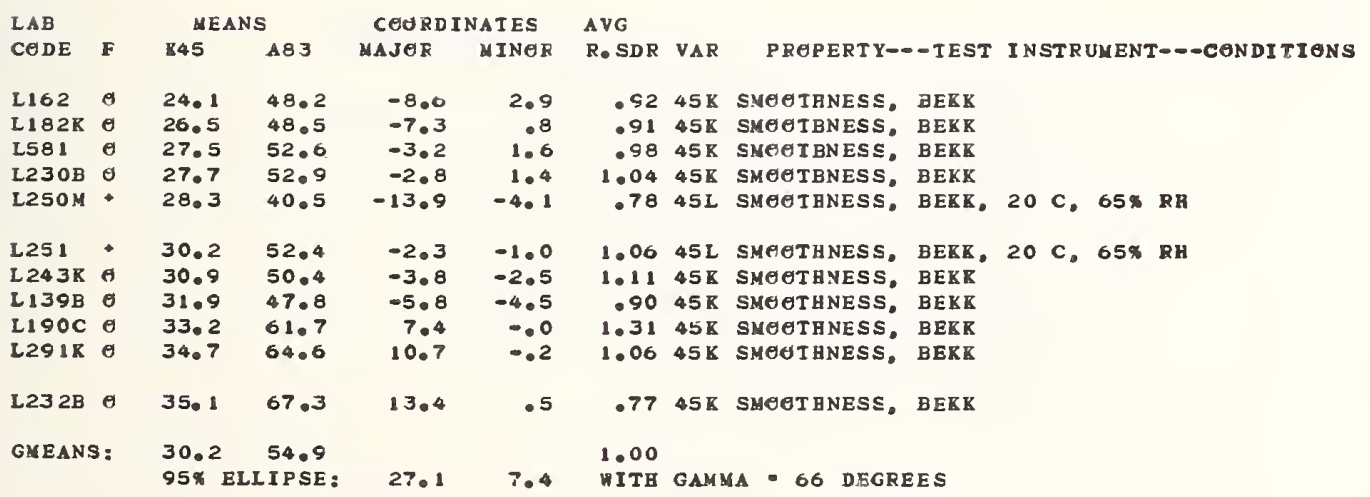




\begin{tabular}{|c|c|c|c|c|c|c|c|c|c|c|c|c|c|}
\hline \multirow{3}{*}{$\begin{array}{l}\text { LAB } \\
\text { CADE }\end{array}$} & \multirow{3}{*}{$\begin{array}{c}\text { SAMPLE } \\
\text { K } 45 \\
\text { MEAN }\end{array}$} & \multicolumn{4}{|c|}{ PRINTING } & SAMPLE & \multicolumn{4}{|c|}{ WUVE ENVELOPE PAPER } & \multirow[t]{2}{*}{ TEST } & \multirow[t]{2}{*}{$D_{0}=$} & \multirow[t]{2}{*}{$=10$} \\
\hline & & 60 GKANS & $S$ PER S & SQUARE M & METER & A 83 & $75 \mathrm{GR}$ & S PER & SQUARE M & METER & & & \\
\hline & & DEV & N. DEV & SDR & $R_{\bullet} S D R$ & MEAN & DEV & N.DEV & SDR & $R_{\bullet} S D R$ & VAR & $\boldsymbol{F}$ & LAB \\
\hline Lico & 193. & -10 & -.67 & 13. & .70 & 91. & -22 & -1.69 & 12. & .65 & $47 b$ & a & L100 \\
\hline L18 $B$ & 230. & 28 & 1.92 & 27. & 1.46 & 122. & 9. & .71 & 26. & 1.47 & $47 B$ & $\boldsymbol{\sigma}$ & $L_{1} 82 B$ \\
\hline L236 & 200 & -2 & -.16 & 13. & .71 & 117. & 40 & .32 & 12. & .66 & 478 & $\theta$ & L236 \\
\hline L.242 & 189. & -14. & -.94 & 18. & .57 & 110 & -3. & -.21 & 9. & .53 & $47 B$ & $\theta$ & L242 \\
\hline L243B & 212 & 10 & .67 & 17. & .90 & $11 \%$ & 1. & .07 & 14. & .77 & $47 B$ & $\theta$ & L243B \\
\hline 1244 & 189. & -13 & -.91 & 20. & 1.10 & 107 & -6 & -.45 & 23. & 1.32 & 478 & $\sigma$ & L244 \\
\hline L248 & 195. & -8 & -.53 & 22. & 1.18 & 136. & 23. & 1.75 & 24. & $1 \cdot 34$ & $47 B$ & $\boldsymbol{\theta}$ & L248 \\
\hline 2333 & 211. & 9. & .61 & 18. & .98 & 106. & -6 & -.49 & 22 & 1.25 & 478 & $\theta$ & L333 \\
\hline
\end{tabular}

GR. MEAN - 203. ML/MIN

GRAND MEAN $=113 . \mathrm{MI} / \mathrm{MIN}$

SD MEANS - 14, ML/MIN

SD GF MEANS - 13. ML/NIN

AVERAGE SDR - 19. HL MIN

AVERAGE SDR *

TEST DETERMINATIONS - 10

8 LABS IN GRAND MEANS TOTAL NUMBER GF LABEFATERTES KEPORTING - 8

8. ML/MIN

Best values: K45 200 milliliter per minute

A83 120 milliliter per minute

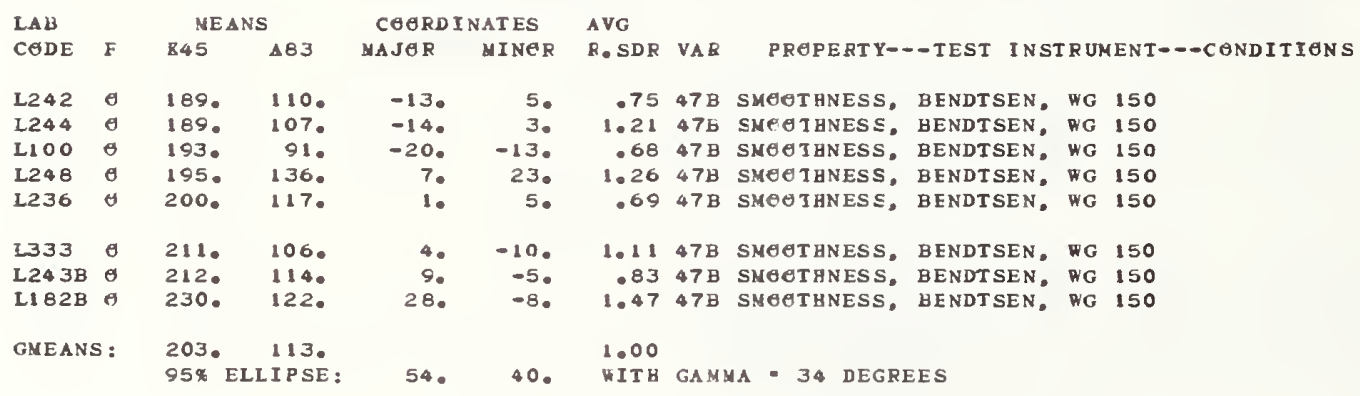




\begin{tabular}{|c|c|c|c|c|c|c|c|c|c|c|c|c|c|}
\hline \multirow{3}{*}{$\begin{array}{l}\text { LAB } \\
\text { CODE }\end{array}$} & SAMPIE & \multicolumn{4}{|c|}{ COATED GLESS } & SAMPLE & \multicolumn{4}{|c|}{ HEAT SET GFFSET BOGE } & \multirow{2}{*}{ TEST } & \multirow{2}{*}{\multicolumn{2}{|c|}{$D_{\bullet} \cdot$}} \\
\hline & E48 & GEA & $S$ PER & SQUARE & & B58 & $76 \mathrm{GR}$ & S PER & SQUARE M & & & & \\
\hline & MEAN & DEV & $N, D E V$ & SDR & R. SDR & MEAN & DEV & N.DEV & SDR & R. 3DR & VAR & $\mathbf{F}$ & LAB \\
\hline L1 26 & 74.9 & $50 \cdot 2$ & 15.40 & .6 & .66 & 42.2 & -19.2 & -2.77 & . 6 & .82 & $56 \mathrm{~K}$ & " & 1126 \\
\hline 1149 & 23.7 & -1.0 & -.29 & .5 & .60 & 54.7 & -6.6 &. .96 & 1.0 & 1.28 & $56 \mathrm{~B}$ & $\theta$ & 2149 \\
\hline L 182 & 28.7 & 4.0 & 1.23 & .6 & .70 & 61.9 & .5 & .08 & $\cdot 2$ & .23 & $56 \mathrm{~K}$ & a & 2182 \\
\hline I213 & 28.4 & 3. 7 & 1.13 & 1.0 & 1.14 & 64.7 & 3.4 & .49 & - 6 & - 86 & $56 \mathrm{E}$ & $\sigma$ & 1213 \\
\hline L2@ 1 & 22.3 & -2.4 & -.73 & 1.3 & 1.53 & 64.9 & 3.5 & .51 & 1.2 & 1.56 & $56 \pi$ & $\theta$ & L29! \\
\hline 1333 & $20 \cdot 5$ & $-4 \cdot 2$ & $-1 \cdot 28$ & - 7 & .89 & $53 \cdot 1$ & $-8 \cdot 3$ & -1.19 & 1. 1 & 1.48 & $56 \mathrm{~K}$ & $\theta$ & 2333 \\
\hline 1339 & 28.2 & 3.5 & 1.09 & .5 & .60 & 71.6 & 10.2 & 1.48 & .5 & .64 & $56 \mathrm{E}$ & A & 1339 \\
\hline 2616 & 23.7 & -1.0 & -.29 & .5 & .60 & 66.7 & 5.4 & .77 & .5 & .67 & $56 \mathrm{~K}$ & $\boldsymbol{\theta}$ & L616 \\
\hline 1643 & 22.0 & -2.7 & -.83 & 1.6 & 1.95 & 53.2 & -8.1 & -1.17 & 1.0 & 1.28 & $56 \mathrm{E}$ & $\theta$ & 1643 \\
\hline
\end{tabular}

GP. MEAN $=24.7 \mathrm{~K} \& \mathrm{~N}$ CNITS

SD MEANS $3.3 \mathrm{~K} \& \mathrm{~N}$ UNITS

AVERAGE SDR =
GRAND NEAN $=61.4 \mathrm{~K}$ \& N UNITS

SD GF MEANS = 6.9 K \& N UNITS

$8 \mathrm{~K} \& \mathrm{~N}$ UNITS

. 81.00

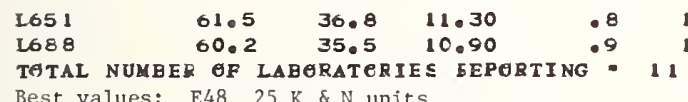

AVERAGE SDR -

TEST DETERMINATIENS -

8 LABS IN GRAND MEANS

Best values: $\begin{array}{lll}\text { E4 } & 25 \mathrm{~K} \& \mathrm{~N} \text { units } \\ \mathrm{B} 58 & 61 \mathrm{~K} \& \mathrm{~N} \text { units }\end{array}$

The following Iaboratories were omitted from the

grand means because of extreme test results; 126.

REPERT NG. 58G

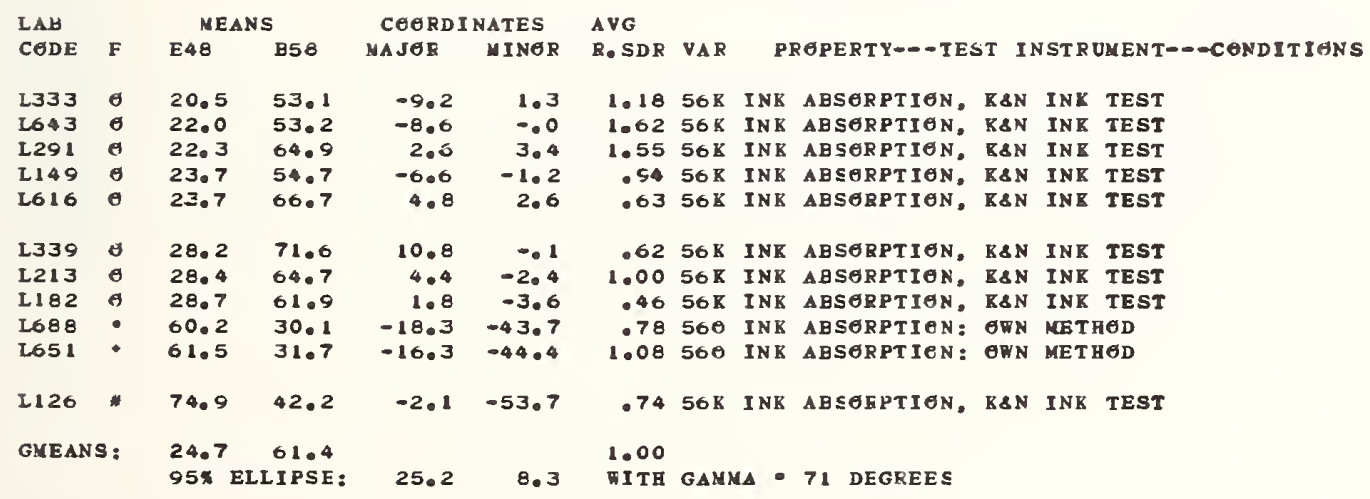

FEBRUARY 1979

OLLABGRATIVE REFERENCE P
ANALYSIS T56-1 TABLE 2

E \& N INE ABSORPTIEN

PEBDUARY 1979 

TAPPI STANDARD TSO9 OS-77

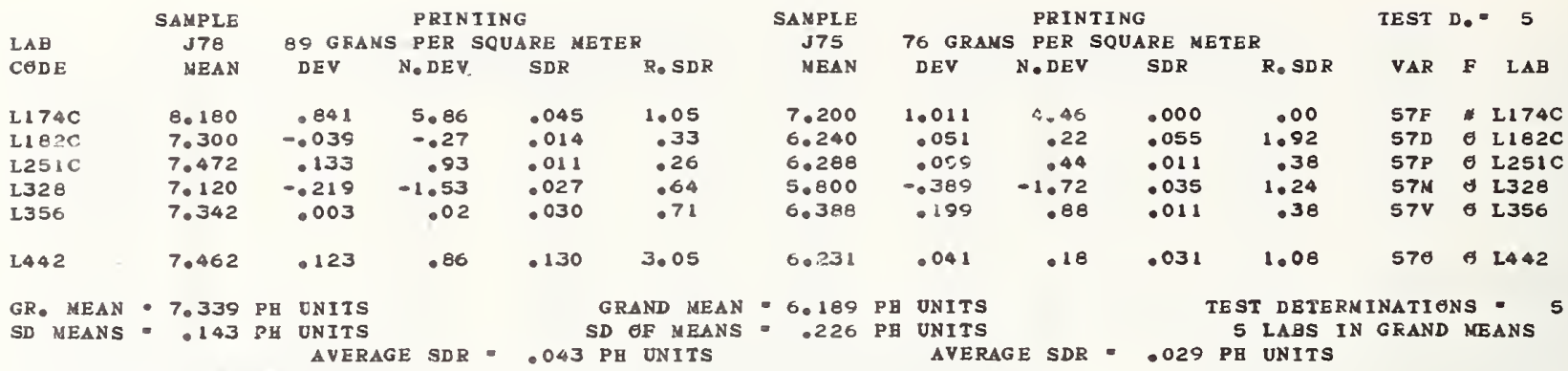

TOTAL NUMBER OF LAEGFATEEIES EEPERTING - 6

Best values: J78 $7.3 \mathrm{pH}$ units

J75 6.2 pH units

The following laboratories were omitted from the

grand means because of extreme test results: $174 \mathrm{C}$.

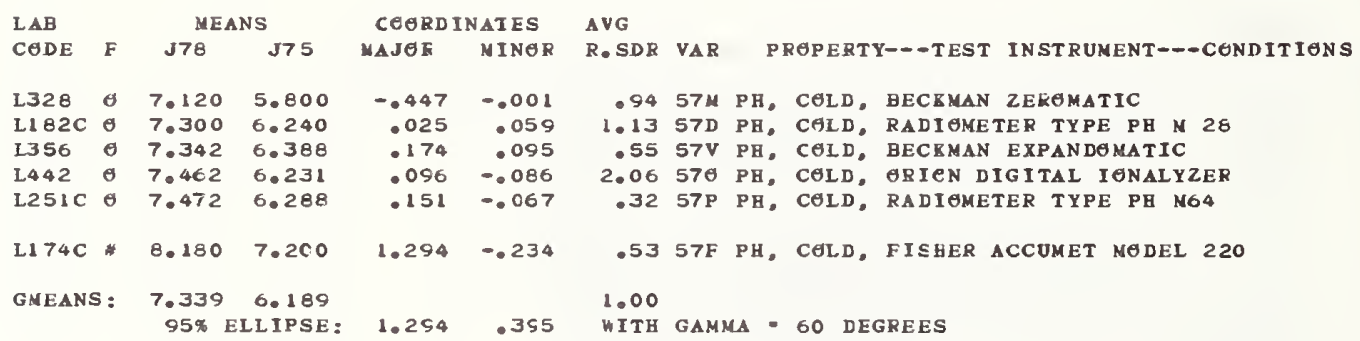


TAPPI STANDARD T 35 O $\$-77$

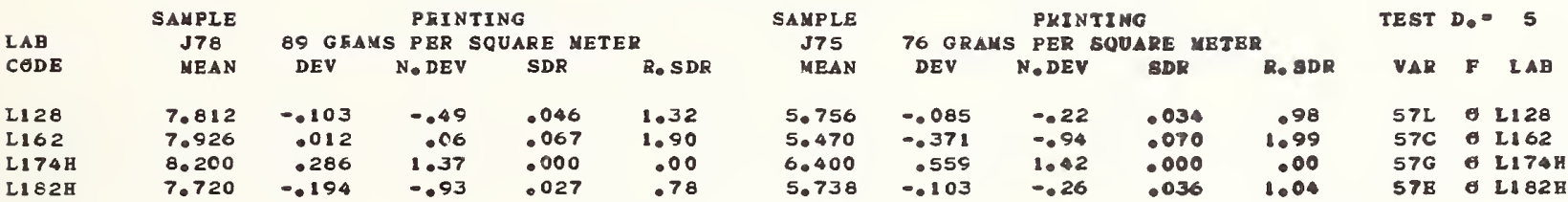

GR. MEAN - 7.914 PH UNIIS GRAND MEAN - 5.841 PH UNITS SD MEANS - 208 PH UNITS SD OF MEANS $=.395$ PH UNITS AVERAGE SDR - .035 PH UNITS AVERAGE SDR . .035 PH UNITS

TOTAL NUMBER GF LABERATERIES EEPGRTING - 4

REPART NG. 58 G

\begin{tabular}{lll} 
LAB & \multicolumn{1}{c}{ MEANS } \\
CODE F J78 & J75
\end{tabular}

L1828 $9.720 \quad 5.738$

I128 7.8125 .756

1162 ศ 7.9265 .470

I 174 B $8.200 \quad 6.400$

G.EANS: 7.9145 .841

95\% ELLIPSE:
TAPPI COLLABURATIVE REFERENCE PREGRAM

ANALYSIS I57-2 TABLE 2

HYDREGEN ION CENCENTRATION (PH), HET

TAPPI STANDARD T435 OS-77

CEORDINAIES AVG

MAJUE MINGE ROSDR VAR PREPERTY---TEST INSTRUMENT---CENDITIENS

$0.173 \quad .137$

$.119 \quad .060$

$0.335=.160$

$.627 \quad-.036$

.9157 E PH, HeT, FADIEMETER TYPE PH M 28

$1.1557 \mathrm{~L} P H$, HबT, $L+N$

$1.9457 \mathrm{C} \mathrm{PH}$, HOT CORNING MUDEL 12 RESEARCH METER

.00570 PH, HAT. FISHER ACCUMET XODEL 220

1.00

WITH GAMMA - 66 DEGREES 
OPACITY (89\% REFLECTANCE BACKING) IN PERCENT

IAPPI STANDA 7 D 425 AS-75, OPACITY AF PAPER (15 DEG•/DIFFUSE, ILLUMINANT A) - BAL TYPE

\begin{tabular}{|c|c|c|c|c|c|c|c|c|c|c|c|c|c|}
\hline \multirow{3}{*}{$\begin{array}{l}\text { LAB } \\
\text { CबDE }\end{array}$} & \multirow{3}{*}{$\begin{array}{c}\text { SAMPLE } \\
\text { E86 } \\
\text { MEAN }\end{array}$} & \multicolumn{4}{|c|}{ BđND } & \multirow{3}{*}{$\begin{array}{c}\text { SAMPLE } \\
\text { B2 I } \\
\text { MEAN }\end{array}$} & \multicolumn{4}{|c|}{ SEMI BLEACHED } & \multirow[t]{2}{*}{ TEST } & \multirow[t]{2}{*}{$D_{\bullet}=$} & \multirow[t]{2}{*}{$=10$} \\
\hline & & 79 G FA & $S$ PER S & SQUARE NE & & & 65 GRA & $S$ PBR S & ARE MI & & & & \\
\hline & & DEV & N. DEV & SDR & $I_{\bullet}$ SDR & & DEV & $N, D E V$ & SDR & R. SDR & VAR & $\boldsymbol{F}$ & LAB \\
\hline L105 & 50.40 & .65 & 1.21 & .59 & 1.66 & 74.00 & 1.64 & $\therefore 54$ & .85 & .84 & $60 \mathrm{H}$ & $\theta$ & L105 \\
\hline LiOS & 89.90 & .15 & .28 & .41 & 1.16 & 72.25 & -011 & -011 & .44 & .44 & $60 \mathrm{~B}$ & A & 1108 \\
\hline $112 B$ & 89.73 & -.02 & -.03 & .35 & 1.00 & 72.49 & $0+3$ & .13 & 1.19 & 1.17 & $60 \mathrm{~B}$ & $\boldsymbol{0}$ & L118 \\
\hline L1 21 & 50.09 & .34 & .63 & .51 & 1.44 & 72.48 & .12 & .12 & 1.56 & 1.53 & $60 \mathrm{~B}$ & $\theta$ & 1221 \\
\hline L122 & 89.41 & -.34 & -.63 & .30 & .86 & 71.38 &. .98 & -.98 & .74 & .73 & $60 D$ & $\theta$ & L 122 \\
\hline L 123 & 89.25 & -.50 & -.92 & .32 & .91 & 72.79 & .43 & .43 & 1.09 & 1.07 & $60=$ & A & $L_{123}$ \\
\hline L124 & 89.39 & -.36 & .66 & .49 & 1.40 & $72 \cdot 63$ & .27 & .27 & .95 & .94 & $60 \mathrm{~B}$ & a & L124 \\
\hline 1125 & 89.27 &. .48 & -.89 & .30 & .85 & 71.02 & $-1 \cdot 34$ & -1.34 & 1.13 & 1.11 & $60 \mathrm{H}$ & a & L125 \\
\hline 4131 & 88.43 & -1.32 & -2.44 & .21 & .58 & 70.67 & -1.65 & -1.69 & .91 & .89 & 6OR & $\theta$ & 2131 \\
\hline$L 132$ & 89.48 & -.27 & -.50 & .25 & .72 & 71.02 & $-1 \cdot 34$ & -1.34 & 1.21 & 1.19 & 605 & $\theta$ & L 132 \\
\hline L1 34 & 89.98 & .23 & .43 & .28 & .79 & 72.84 & .48 & .48 & 1.05 & 1.03 & $60 R$ & A & I 134 \\
\hline L1 36 & 89.40 & -.35 & -.65 & .33 & .94 & 72.41 & .05 & .05 & 1.00 & .98 & $60 \mathrm{~B}$ & A & L1 36 \\
\hline 1139 & 89.50 & -.25 & -.46 & .35 &.$\$ 9$ & 71.68 & .068 &. .68 & .85 & .84 & 603 & $\sigma$ & 2139 \\
\hline LI $48 \mathrm{H}$ & 89.29 & -.46 & -.85 & .30 & - 84 & 71.20 & -1.16 & -1.16 & 1.22 & 1.20 & $60 \mathrm{H}$ & 6 & $L_{148 I}$ \\
\hline L150 & 50.20 & .45 & .84 & .42 & 1.20 & 74.10 & 1.74 & 1.74 & .81 & .80 & $60 \mathrm{~B}$ & $\boldsymbol{\theta}$ & L150 \\
\hline L152 & $\$ 0.05$ & .30 & .56 & .23 & .64 & 73.08 & .72 & .72 & .88 & .86 & $60 \mathrm{~B}$ & $\theta$ & L152 \\
\hline 153 & 89.95 & .20 & .37 & .64 & 1.83 & 72.55 & .59 & .59 & .69 & .67 & 608 & $\theta$ & $L 153$ \\
\hline L157 & $\$ 0.35$ & .60 & 1.12 & .41 & 1.17 & 72.95 & .59 & .59 & 1.82 & 1.79 & 608 & $\theta$ & L 157 \\
\hline L158 & 90.38 & .63 & 1.17 & .50 & 1.41 & 73.80 & 1.44 & 1.44 & 1.19 & 1.17 & $60 D$ & $\theta$ & $L 158$ \\
\hline L159 & 89.61 & -.14 & -.26 & .20 & .57 & 71.04 & -1.32 & -1.32 & 1.03 & 1.01 & GOR & $\sigma$ & L159 \\
\hline L162 & 50.01 & .26 & .49 & .33 & . 93 & $72 \cdot 22$ &. .14 & -.14 & .89 & .87 & $60 \mathrm{~W}$ & e & L162 \\
\hline L166 & 88.76 & -.99 & -1.83 & .58 & 1.63 & 71.37 & -.95 & -.99 & 1.57 & 1.54 & $60 \mathrm{~B}$ & $\theta$ & $L_{166}$ \\
\hline L1734 & 90.30 & .55 & 1.02 & .48 & 1.37 & 72.20 &. .16 & -.16 & .52 & .90 & $60 \mathrm{~B}$ & $\theta$ & 21734 \\
\hline L190C & 89.08 & -.67 & $-1 \cdot 24$ & .34 & .97 & $70 \cdot 16$ & -2.20 & -2.21 & 1.41 & 1.39 & $60 \mathrm{~B}$ & $\theta$ & L $190 \mathrm{C}$ \\
\hline LISOR & 89.77 & .02 & .04 & .37 & 1.04 & 72.64 & .28 & .28 & 1.21 & 1.19 & $60 \mathrm{~B}$ & $\theta$ & L $190 R$ \\
\hline L206 & 50.00 & .25 & .47 & .34 & .95 & $73 \cdot 38$ & 1.02 & 1.02 & 1.29 & 1.26 & $60 \mathrm{~B}$ & $\theta$ & L206 \\
\hline L2 $10 \mathrm{~B}$ & 89.93 & .18 & .34 & .29 & .82 & 73.21 & .85 & .85 & .93 & .92 & $60 \mathrm{~B}$ & $\theta$ & L2 $10 \mathrm{~B}$ \\
\hline L210D & 90.14 & .35 & .73 & .35 & 1.00 & 73.07 & .71 & .71 & .79 & .78 & $60 D$ & $\theta$ & $L 210 D$ \\
\hline $\mathrm{L} 211 \mathrm{~s}$ & 28.42 & -1.33 & -2.46 & .38 & 1.08 & 69.68 & -2.68 & -2.69 & 1.21 & 1.19 & $60 R$ & . & L211s \\
\hline L2 12 & 50.50 & .75 & 1.40 & .71 & 2.01 & 72.80 & .44 & .44 & .79 & .78 & $60 \mathrm{~B}$ & $\theta$ & L212 \\
\hline L2 13 & So.11 & .36 & .67 & .45 & 1.26 & 72.84 & .48 & .48 & .91 & .89 & $60 \mathrm{~B}$ & $A$ & L2 13 \\
\hline L22 $3 B$ & 90.11 & - $3 \epsilon$ & .67 & .39 & 1.10 & 72.41 & .05 & .05 & 1.40 & 1.37 & $60 B$ & $\theta$ & L2238 \\
\hline L225 & 90.91 & 1.16 & 2.16 & .64 & 1.82 & 74.62 & 2.26 & 2.26 & 1.11 & 1.09 & $60 \mathrm{~B}$ & A & L225 \\
\hline L226B & 88.86 & -.85 & -1.65 & .30 & .86 & 70.84 & -1.52 & -1.52 & .74 & .73 & $60 \mathrm{~B}$ & $\theta$ & L226B \\
\hline L22 8 & 89.44 & -.31 & -.57 & .27 & .76 & 72.35 & .01 & -.01 & .91 & .90 & $60 \mathrm{H}$ & $\theta$ & L228 \\
\hline L230 & 89.92 & .17 & .32 & .18 & .51 & 72.02 & -.34 & .34 & .50 & .49 & 608 & $\boldsymbol{\sigma}$ & L2 30 \\
\hline L236B & 89.02 & -.73 & $-1 \cdot 35$ & .64 & 1.81 & $71 \cdot 22$ & -1.14 & -1.14 & .45 & .49 & $60 \mathrm{~B}$ & $\theta$ & L 236 B \\
\hline L238A & $88 \cdot 38$ & -1.37 & -2.54 & .24 & .69 & 70.79 & -1.57 & -1.57 & .54 & .53 & $60 \mathrm{R}$ & * & L238A \\
\hline L241 & 89.55 & $-.2 c$ & -.37 & .18 & .52 & 72.77 & .41 & .41 & 1.06 & 1.04 & $60 \mathrm{~B}$ & e & L241 \\
\hline L243 & 29.56 & .15 & -.35 & .44 & 1.24 & 72.01 &. .35 & -.35 & .74 & .73 & $60 \mathrm{~B}$ & $\boldsymbol{\theta}$ & L243 \\
\hline L254 & 90.20 & .45 & .84 & .41 & 1.17 & 72.06 & -.30 & -.30 & 1.09 & 1.07 & $60 \mathrm{H}$ & $\theta$ & L254 \\
\hline L255 & 89.87 & .12 & .23 & .21 & .60 & 72.00 & -.36 & -.36 & .65 & .64 & $60 B$ & $\theta$ & 1255 \\
\hline L259 & 89.59 & .24 & .45 & .45 & 1.28 & 73.10 & .74 & .74 & .84 & .83 & $60 B$ & $\theta$ & L259 \\
\hline L261 & 50.47 & .72 & 1.34 & .35 & 1.00 & $73 \cdot 20$ & .84 & .84 & 1.06 & 1.04 & $60 B$ & $\theta$ & L261 \\
\hline L262 & 50.36 & .61 & 1.14 & .27 & .77 & 72.80 & .44 & .44 & .88 & .87 & $60 R$ & $a$ & L262 \\
\hline L275 & 89.34 & -.41 & -.76 & .37 & 1.05 & 71.53 &. .83 &. .83 & .85 & .83 & $60 R$ & $\theta$ & L 275 \\
\hline L278 & 50.00 & .25 & .47 & .59 & 1.67 & 72.93 & .57 & .57 & .90 & .89 & $60 B$ & A & L278 \\
\hline 1281 & 89.77 & .02 & .04 & .24 & .68 & 72.92 & .56 & .56 & .91 & .89 & $60 \mathrm{D}$ & $\theta$ & L281 \\
\hline L285D & 89.52 & -.23 &. .42 & .28 & .80 & 72.08 & -.28 &. .28 & 1.38 & 1.35 & $60 D$ & $\boldsymbol{\theta}$ & L285D \\
\hline L285R & 89.14 &. .61 & $-1 \cdot 13$ & .21 & .60 & 71.26 & -1.10 & -1.10 & .88 & .86 & $60 R$ & $\theta$ & L2 $85 R$ \\
\hline L28 8 & 90.03 & .28 & .52 & .46 & 1.30 & 72.13 & -.23 & -.23 & 1.12 & 1.10 & 6OD & $\theta$ & L288 \\
\hline L. 301 & 89.61 & -.14 & -.26 & .23 & .66 & 72.22 & -.14 & .14 & .71 & .70 & $60 B$ & $\theta$ & 1301 \\
\hline L305 & 89.67 & -.08 & -114 & .18 & .52 & 71.52 & -.84 & -.84 & .87 & .85 & $60 \mathrm{R}$ & $\theta$ & 2305 \\
\hline L308 & 50.45 & .70 & 1.30 & .22 & .62 & $73 \cdot 13$ & .77 & .77 & 1.35 & 1.33 & $60 \mathrm{H}$ & $\boldsymbol{\theta}$ & L308 \\
\hline 1315 & 89.21 & -.54 & -1.00 & .35 & 1.00 & $71 \cdot 26$ & -1.10 & -1.10 & 1.42 & 1.39 & $60 \mathrm{D}$ & ब & L315 \\
\hline 2317 & 50.04 & .29 & .54 & .30 & .86 & 72.55 & .19 & .19 & .63 & .62 & $60 B$ & $\theta$ & L317 \\
\hline 1318 & 90.05 & .30 & .56 & .44 & 1.24 & $73 \cdot 35$ & .99 & .99 & .75 & .73 & $60 \mathrm{~B}$ & $\theta$ & L318 \\
\hline L323 & 89.92 & .17 & .32 & .36 & 1.01 & $72 \cdot 36$ & -.00 & -000 & 1.14 & 1.12 & $60 \%$ & $\boldsymbol{\sigma}$ & L.323 \\
\hline $\mathbf{L} 326$ & 89.60 &. .15 & -.27 & .44 & 1.25 & 72.84 & .48 & .48 & 1.71 & 1.68 & $60 B$ & $\theta$ & L326 \\
\hline L328 & 50.60 & .85 & 1.58 & .70 & 1.58 & 72.60 & .24 & .24 & 1.43 & 1.41 & $60 B$ & $\theta$ & L328 \\
\hline 1339 & 89.00 & -.75 & -1.39 & .47 & 1.34 & 90.00 & 17.64 & 17.67 & .00 & .00 & $60 \mathrm{~B}$ & $*$ & L339 \\
\hline 1349 & 89.75 & .00 & .00 & .19 & .54 & 72.80 & .44 & .44 & .75 & .74 & 600 & $\sigma$ & 1349 \\
\hline 1352 & 89.18 & -.57 & -1.05 & .22 & .62 & 72.10 &. .26 & -.26 & .69 & .68 & $60 R$ & $\theta$ & L352 \\
\hline L354 & 89.00 &. .75 & -1.39 & .00 & .00 & 71.20 & -1.16 & -1.16 & .79 & .78 & $60 \mathrm{~B}$ & $\boldsymbol{\theta}$ & L354 \\
\hline L390 & 89.92 & .17 & .32 & .27 & .75 & 73.92 & 1.56 & 1.56 & 1.92 & 1.89 & $60 \mathrm{~B}$ & $\boldsymbol{\theta}$ & L390 \\
\hline
\end{tabular}



GPACITY (89\% REFLECTANCE BACEING) IN PERCENT TAPPI STANDAED T425 ES-75, GPACITY GF PAPER (15 DEG./DIFFUSE, ILLUMINANT AD - BAL TYPE

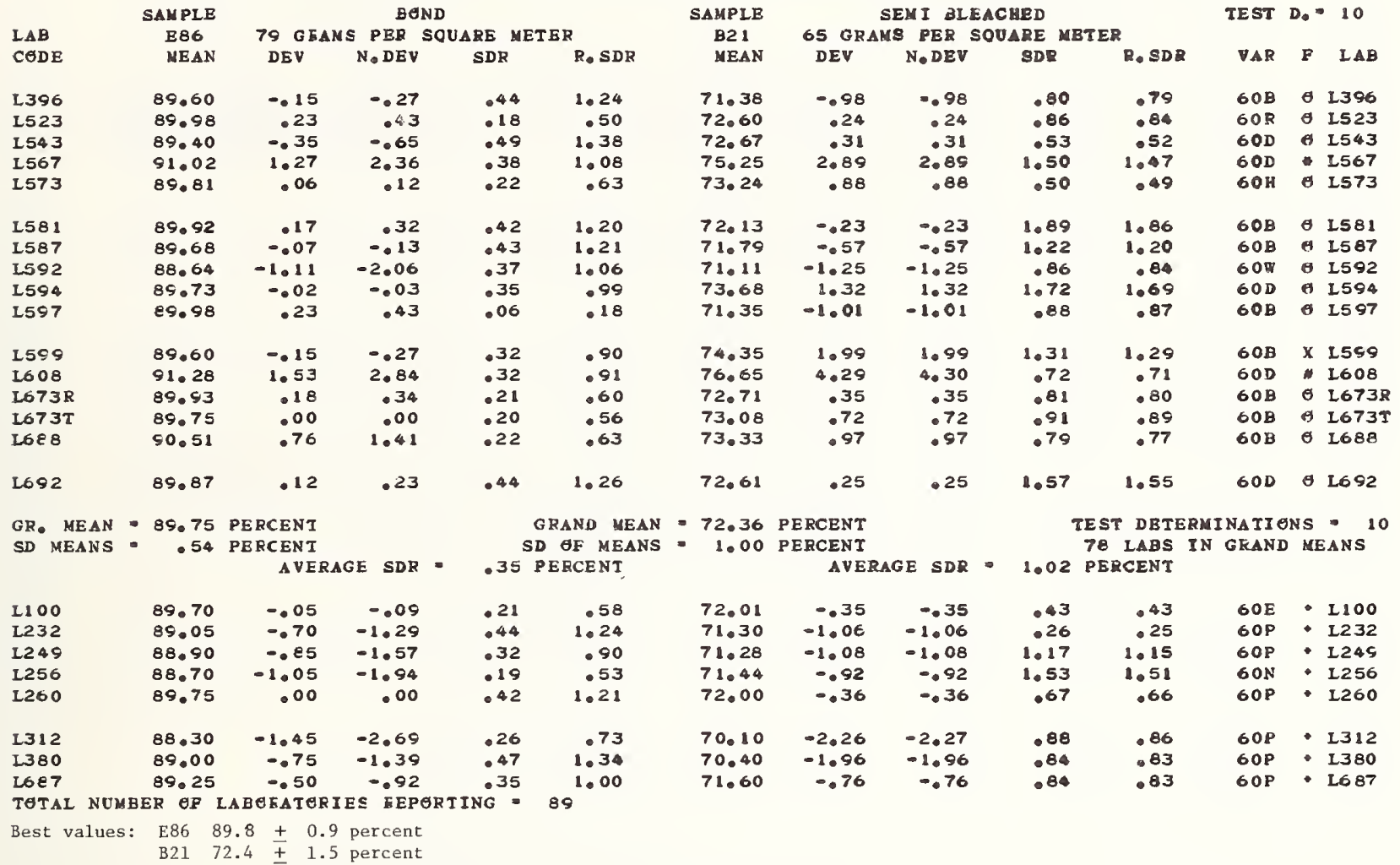

The following laboratories were omitted from the grand means because of extreme test results: 339 , 608. 
OPACIIY (89\% REFLECTANCE BACKING) IN PERCENT

TAPPI STANDAFD T425 OS-75, OPACITY OF PAPER (15 DEG /DIFFUSE, ILLUMINANT A) - B\&L TYPE

\begin{tabular}{|c|c|c|c|c|c|c|c|c|c|c|c|c|c|}
\hline \multirow{2}{*}{$\begin{array}{l}\text { LAB } \\
\text { CODE }\end{array}$} & \multirow[b]{2}{*}{$\mathbf{F}$} & \multicolumn{2}{|c|}{ MEANS } & \multicolumn{2}{|c|}{ CUERDINATES } & \multicolumn{2}{|l|}{ AVG } & \multirow{2}{*}{\multicolumn{5}{|c|}{ PREPERTY---TEST INSTRUMENT---CENDITIONS }} & \\
\hline & & E86 & B2 1 & MAJOF & MINER & E.SDR & VAR & & & & & & \\
\hline L312 & $\bullet$ & 88.30 & $70 \cdot 10$ & -2.66 & .35 & .80 & $60 P$ & OPACI IY & ( WHITE & BACKING), & PBeTEVeLt & & \\
\hline L238A & $*$ & $88 \cdot 38$ & 70.75 & -2.00 & .57 & .61 & $60 R$ & OPACI TY & ( FEITE & BACKING ; & IHEING-ALBERT & ( FERNERLY & SRL) \\
\hline L2:1S & $\leftarrow$ & 88.42 & 69.68 & -2.99 & .07 & 1.14 & $60 \mathrm{R}$ & APACITY & ( PBITE & BAC $(I N G)$, & TH\#ING-ALBERT & ( FERMBRLY & SRL) \\
\hline L 131 & 6 & $88 \cdot 43$ & 70.67 & -2.09 & .48 & .74 & $60 \mathrm{R}$ & OPACITY & ( WHITE & 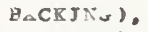 & THWI NG-ALBERT & (FERMERLY & SRL ) \\
\hline L5S 2 & $\theta$ & 88.64 & 71.11 & -1.60 & .47 & .95 & $60 \%$ & OPACITY & ( FESTE & MAKI NG ), & HUYGEN, DIGITAL & & \\
\hline L256 & $\bullet$ & 88.70 & 71.44 & $-1 \cdot 28$ & .56 & 1.02 & $60 \mathrm{~N}$ & OPACI IY & 、 कHIT工 & BAC K I NG ). & HONTER & & \\
\hline L 166 & $\theta$ & 88.76 & $71 \cdot 37$ & -1.32 & .48 & 1.59 & $60 B$ & OPACITY & ( $\mathrm{AHTTE}$ & BACRING). & BALSCH - LONB & & \\
\hline L 226B & $\theta$ & 88.86 & 70.84 & $-1 \cdot 75$ & .16 & .79 & $60 \mathrm{~B}$ & OPACITY & ( WHITE & BACKING ), & BAUSCB - LANB & & \\
\hline L249 & + & 88.90 & $71 \cdot 28$ & $-1 \cdot 34$ & $\cdot 31$ & 1.03 & $60 P$ & APACITY & ( WHITE & BACKING), & PBeT & & \\
\hline 1380 & + & 89.00 & 70.40 & -2.09 & .015 & 1.08 & 6OP & OPACITY & ( พHITE & BAC XING ), & PHET TEVELT & & \\
\hline $\mathrm{L} 354$ & $\boldsymbol{\theta}$ & 89.00 & $71 \cdot 20$ & -1.37 & .19 & $\cdot 39$ & $60 B$ & OPACI TY & ( HBITE & BACK ING ), & BAUSCH * LGMB & & \\
\hline 1339 & \# & 89.00 & 90.00 & $15 \cdot 66$ & 8.15 & .67 & $60 \mathrm{~B}$ & OPACITY & ( & BACKING), & BAUSCH * LONB & & \\
\hline L236B & $\boldsymbol{\theta}$ & 89.02 & $71 \cdot 22$ & $-1 \cdot 34$ & .18 & 1.15 & $60 \mathrm{~B}$ & OPACITY & ( FEITE & BACKING), & BAUSCH * LOMB & & \\
\hline L232 & $\bullet$ & 85.05 & $71 \cdot 30$ & $-1 \cdot 20$ & .18 & .75 & $60 P$ & GPACI IY & ( जHITE & BACKING), & PHETEVGLT & & \\
\hline L19OC & $\theta$ & 89.08 & $70 \cdot 16$ & -2.28 &. .33 & 1.18 & 608 & OPACITY & ( VBITE & BACKING). & BAUSCH * LEMB & & \\
\hline L285R & $\theta$ & $89 \cdot 14$ & $71 \cdot 26$ & $-1 \cdot 25$ & .08 & .73 & $60 \mathrm{R}$ & OPACITY & ( WHITE & BACKING ), & THWI NG-A LBERT & ( F ORMERLY & SRL ) \\
\hline L352 & $\theta$ & $89 \cdot 18$ & $72 \cdot 10$ & .48 & .40 & .65 & $60 \mathrm{R}$ & OPACITY & ( ИНITE & BACKING ), & THWI NG -AL BERT & ( FGRNERLY & SRL) \\
\hline L315 & $\theta$ & 89.21 & $71 \cdot 26$ & $-1 \cdot 23$ & .02 & 1.19 & $60 \mathrm{D}$ & OPACI IY & ( REITE & BACKING ), & $H N L=2$ & & \\
\hline 1687 & 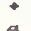 & 89.25 & 71.60 &. .90 & .13 & .92 & 60P & OPAC I TY & ( WВ I TE & BACKING ), & PHATEVEIT & & \\
\hline L123 & $\boldsymbol{\sigma}$ & 89.25 & 72.79 & $\cdot 18$ & .63 & .99 & $60 \mathrm{~W}$ & EPACI TY & ( ABITE & BACKING), & BUYGEN, DIGITAL & & \\
\hline L 125 & $\theta$ & 89.27 & 71.02 & -1.42 &. .13 & .98 & $60 \mathrm{H}$ & OPACI TY & ( ФHITE & BAC K ING), & BUYGEN & & \\
\hline L $148 \mathrm{H}$ & e & $89 \cdot 29$ & $71 \cdot 20$ & -1.25 & -.08 & 1.02 & $60 \mathrm{H}$ & OPACI TY & ( WHITE & BACEING), & HUYGEN & & \\
\hline L 275 & $\theta$ & $89 \cdot 34$ & 71.53 &. .93 & .02 & .94 & $60 \mathrm{R}$ & OPACITY & ( WBITE & BACKING ), & THWI NG - AL BERT & ( FORMERLY & SRL ) \\
\hline L1 24 & $\theta$ & 89.39 & 72.63 & .09 & .44 & 1.17 & $60 \mathrm{~B}$ & OPACI IY & ( НHITE & BACKING ), & BAUSCH * LOMB & & \\
\hline 2543 & $\theta$ & 89.40 & 72.67 & .13 & .45 & .95 & 601 & OPACITY & ( WBITE & BACEING), & $B N L-2$ & & \\
\hline L136 & $\theta$ & 89.40 & $72 . \div i$ & -.10 & .34 & .96 & $60 \mathrm{H}$ & OPACI TY & ( WHITE & BACKING ), & HUYGEN & & \\
\hline L122 & $\theta$ & 89.41 & $71 \cdot 38$ & -1.03 & -.11 & .79 & GOD & OPACITY & ( WHITE & BAC KING), & $B N L=2$ & & \\
\hline L228 & $\theta$ & 89.44 & $72 \cdot 35$ & .14 & .27 & $\bullet 83$ & $60 \mathrm{H}$ & APACITY & ( WBITE & BACRING), & HUYGEN & & \\
\hline L132 & $\boldsymbol{\theta}$ & $89 \cdot 48$ & 71.02 & $-1 \cdot 33$ & -.33 & .95 & $60 \mathrm{~B}$ & OPACI IY & ( WBITE & BACKING), & $B \triangle U S C H+\angle O H B$ & & \\
\hline L139 & $\theta$ & 89.50 & 71.68 & -.72 & -.06 & .91 & 608 & OPACITY & ( WHITE & HACKING). & BAUSCB + LEMB & & \\
\hline L285D & $\theta$ & 89.52 & 72.08 & -.35 & .09 & 1.08 & $60 \mathrm{D}$ & OPACITY & (WHITE & ( $A$ ACKING), & $B N L=2$ & & \\
\hline L241 & $\theta$ & 89.55 & 72.77 & .25 & . 35 & .78 & $60 \mathrm{~B}$ & OPACITY & ( WHITE & BACKING). & HAUSCH * LOMB & & \\
\hline L243 & $\boldsymbol{\theta}$ & 89.56 & 72.01 & -.40 & .02 & .99 & $60 \mathrm{~B}$ & EPACI TY & ( W月ITE & BACKING). & BAUSCB * LGNB & & \\
\hline L599 & $\mathbf{X}$ & 89.60 & 74.35 & 1.74 & .98 & 1.09 & $60 \mathrm{~B}$ & EPACITY & ( WHITE & BACKING ), & BAUSCB + LONB & & \\
\hline 2396 & $\theta$ & 89.60 & $71 \cdot 38$ & -.95 & -.28 & 1.01 & $60 B$ & OPACITY & ( WHITE & BACKING). & BAUSCB + LENB & & \\
\hline L326 & $\theta$ & 89.60 & $72 \cdot 84$ & .37 & $\cdot 34$ & 1.46 & $60 \mathrm{~B}$ & OPACITY & ( DHITE & BACKING ), & BAUSCH — LOMB & & \\
\hline L 159 & $\theta$ & 85.61 & 71.04 & $-1 \cdot 26$ & -.43 & .79 & $60 R$ & OPACI TY & ( WITE & HACKING). & THW I NG-ALBER T & (FORNERLY & SRL ) \\
\hline 1301 & $\theta$ & 89.61 & 72.22 &. .19 & .07 & .68 & $60 \mathrm{~B}$ & G?ACITY & ( WHITE & BACKING). & HAUSCH & & \\
\hline 1305 & $\theta$ & 89.67 & 71.52 & -.80 & -.29 & .69 & $60 \mathrm{R}$ & OPACITY & ( WHITE & BACKING), & THWING-ALBERT & ( FER VERLY & SRL ) \\
\hline L587 & $\theta$ & 89.68 & 71.79 &. .55 & -.18 & 1.20 & $60 \mathrm{~B}$ & APACI TY & ( WEITE & BACKING ), & BAUSCB — LONB & & \\
\hline L100 & + & 89.70 & 72.01 & -.34 & -.11 & .50 & $6 \mathrm{CE}$ & $\Theta P A C I T Y$ & ( WHITE & BACKING), & ZEISS ELREPBO, & $F M Y-C(10)$ & FILTE \\
\hline L118 & $\theta$ & 89.73 & 72.49 & .11 & .07 & 1.08 & $60 \mathrm{~B}$ & EPACITY & ( AHITE & BACKING), & BAUSCH * LONB & & \\
\hline 1594 & $\boldsymbol{\sigma}$ & 89.73 & 73.68 & 1.19 & .57 & $1 \cdot 34$ & 60D & OPACITY & ( WHI TE & BACKING), & BNL - 2 & & \\
\hline L260 & $\bullet$ & 89.75 & 72.00 &. .33 &. .15 & .93 & OOP & EPACITY & ( WHITE & BACKING ), & PHOTOVOLT & & \\
\hline L673T & $\theta$ & 89.75 & 73.08 & .65 & .30 & .72 & 605 & ӨPACI TY & ( WH I TE & BACKING ), & BAUSCB - LOMB & & \\
\hline 1349 & $\theta$ & 89.75 & 72.80 & .40 & .18 & .64 & $60 \mathrm{D}$ & OPACITY & ( ПНITE & BACKING). & BNL-2 & & \\
\hline L $19 \mathrm{CR}$ & $\theta$ & 89.77 & 72.64 & $.2 \epsilon$ & .10 & 1.11 & $60 \mathrm{~B}$ & OPACI TY & ( АHITE & BACK ING ). & BAUSCH + LOVB & & \\
\hline L281 & $\theta$ & 89.77 & 72.92 & .52 & .22 & .79 & $60 \mathrm{D}$ & OPACITY & ( WHITE & BACKING ), & $B N L-2$ & & \\
\hline$L 573$ & $\theta$ & 89.81 & 73.24 & .82 & .32 & .56 & $\epsilon \mathrm{OH}$ & OPACITY & ( WHITE & BACKING), & HUYGEN & & \\
\hline L255 & $\boldsymbol{\theta}$ & 89.87 & 72.00 & $-.2 \varepsilon$ & -.26 & .62 & $60 \mathrm{H}$ & OPACITY & ( WHI TE & BACKING ), & BAUSCB - LONB & & \\
\hline 1692 & $\theta$ & 89.87 & 72.61 & .28 & -.01 & 1.40 & 6OD & GPACI TY & ( ИНITE & BACK ING ), & BNL-2 & & \\
\hline 2108 & $\theta$ & 89.90 & $72 \cdot 25$ & -.04 & -.18 & .80 & 6013 & OPACI TY & ( WHITE & BACKING), & BAUSCH * LENB & & \\
\hline L58 1 & $\boldsymbol{\theta}$ & 89.92 & 72.13 & -.14 & -.25 & 1.53 & $60 \mathrm{~B}$ & OPACI TY & ( WHITE & BACKING), & ВAUSCH LOMB & & \\
\hline L390 & $\boldsymbol{\theta}$ & 89.92 & 73.92 & 1.49 & .50 & 1.32 & $60 \mathrm{H}$ & OPACI TY & ( МHI TE & BACKING), & BAUSCH + LOMB & & \\
\hline L230 & $\boldsymbol{\theta}$ & 89.92 & 72.02 & -.24 & -.30 & .50 & $60 B$ & OPACITY & (WHITE & BACKING I, & BAUSCE - LOMB & & \\
\hline L323 & $\theta$ & 89.92 & 72.36 & .07 & -.16 & 1.06 & $60 \mathrm{~W}$ & OPACITY & ( W I TE & BACKING), & HUYGEN, DIGITAL & & \\
\hline L673R & $\boldsymbol{\theta}$ & 89.93 & 72.71 & .39 &. .02 & .70 & $60 \mathrm{~B}$ & OPACI TY & ( & BACKING), & BAUSCB • LOMH & & \\
\hline L210B & $\boldsymbol{\theta}$ & 89.93 & 73.21 & - 25 & .19 & .87 & $60 \mathrm{~b}$ & OPACI TY & ( WHITE & BACKING), & BAUSCH • LOMH & & \\
\hline 1153 & $\boldsymbol{\theta}$ & 89.95 & 72.95 & .62 & .07 & 1.25 & $60 \mathrm{H}$ & OPACI TY & ( WHITE & BACKING), & BAUSCH • LOME & & \\
\hline $\mathrm{L} 134$ & 0 & 89.58 & 72.84 & .53 & -.01 & .91 & $60 \mathrm{R}$ & OPACITY & ( WHITE & BACKING ), & TEW I NG-AL BER T & ( FORMERLY & SRL ) \\
\hline 1597 & 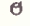 & 89.98 & $71 \cdot 35$ & -.82 & -.64 & .52 & $60 \mathrm{~B}$ & OPACI TY & ( $\mathrm{MHITE}$ & BACXING), & BAOSCE — LAMB & & \\
\hline L523 & $\theta$ & 89.98 & 72.60 & .31 & -.11 & .67 & $60 \mathrm{R}$ & EPACITY & ( WHITE & BACKING). & THWING-AL BERT & (FORNERLY & SRL) \\
\hline L259 & $\boldsymbol{\sigma}$ & 89.99 & $73 \cdot 10$ & .77 & .09 & 1.05 & $60 \mathrm{~B}$ & EPACITY & ( WHITE & BACKING), & BAUSCH $\bullet$ LAMB & & \\
\hline L206 & $\boldsymbol{\theta}$ & 90.00 & $73 \cdot 38$ & 1.03 & .20 & 1.11 & $60 \mathrm{~B}$ & BPACITY & ( WHITE & BACKING), & BAUSCH • LOMB & & \\
\hline 1278 & $\theta$ & 50.00 & 72.93 & .62 & .01 & 1.28 & $60 \mathrm{~B}$ & OPACI IY & ( WHITE & BACKING), & BAUSCB LEMB & & \\
\hline
\end{tabular}


OPACITY ( B9\% REFIECTANCE BACKING) IN PERCENT

IAPPI STANDARD T425 OS-75, OPACITY OF PAPER (15 DEG,/DIFFUSE. ILLUMINANT A) - BAL TYPE

\begin{tabular}{|c|c|c|c|c|c|c|c|c|c|c|c|c|c|}
\hline \multirow{3}{*}{$\begin{array}{l}L_{A B} \\
C A D E \\
1162\end{array}$} & \multirow{3}{*}{$\begin{array}{l}F \\
\theta\end{array}$} & \multicolumn{2}{|c|}{ MEANS } & \multicolumn{2}{|c|}{ CEORDINATES } & \multirow{2}{*}{$\begin{array}{l}\text { AVG } \\
R_{\bullet} \text { SDR }\end{array}$} & \multirow[b]{2}{*}{ VAK } & \multirow{2}{*}{\multicolumn{2}{|c|}{ PROPERTY $=-$ IEST }} & \multirow{2}{*}{\multicolumn{3}{|c|}{ I INSTRUENT $-\infty$ CENDITIENS }} & \\
\hline & & E86 & E21 & MAJ日R & MINGR & & & & & & & & \\
\hline & & 90.01 & 72.22 & .02 & -.30 & .90 & $60 \mathrm{~W}$ & OPACITY & ( DHITE & BACKING), & HUYGEN, & DIGITAL & \\
\hline L288 & A & 90.03 & $72 \cdot 13$ & -.09 &. .35 & 1.20 & $60 D$ & APACITY & ( DEITE & BACKING), & $B N L=2$ & & \\
\hline 2317 & A & 90.04 & 72.55 & .29 & $\because 18$ & .74 & $60 \mathrm{~B}$ & OPACITY & ( ВHITE & BACXING), & BAUSCB & - Lөмв & \\
\hline L152 & $\theta$ & 90.05 & 73.08 & .78 & .03 & .75 & $60 \mathrm{~B}$ & OPACITY & ( ИHITE & BACKING), & BAUSCH & - цөмв & \\
\hline L318 & $\boldsymbol{\theta}$ & 90.05 & 73.35 & 1.02 & .15 & .99 & 608 & OPACI TY & ( WBI TE & BACKING), & BAUSCE & - LฮบB & \\
\hline I 121 & A & 90.09 & 72.48 & .25 &. .26 & 1.48 & $60 \mathrm{~B}$ & GPACITY & ( WBITE & BACKING), & BAUSCE & - LeNB & \\
\hline L223B & ฮ & 90.11 & 72.41 & .20 &. .31 & 1.24 & $60 \mathrm{~B}$ & OPACITY & ( พBITE & BACKING), & BA USCE & - LeMB & \\
\hline 1213 & $\sigma$ & 90.11 & 72.84 & .55 & .013 & 1.08 & $60 \mathrm{~B}$ & GPACI TY & ( सIIE & BACKING), & BAUSCR & - LAMB & \\
\hline L21OD & $\sigma$ & 90.14 & 73.07 & .81 & .05 & .89 & 6OD & GPACITY & ( \$HITE & BACK ING), & $B N L=2$ & & \\
\hline L150 & $\theta$ & 90.20 & $74 \cdot 10$ & 1.77 & .33 & 1.00 & $60 \mathrm{~B}$ & OPACITY & ( VBITE & BACKING), & BAUSCH & - LबMB & \\
\hline L254 & A & $\$ 0.20$ & 72.06 & .08 &. .54 & 1.12 & 608 & OPACITY & ( WBITE & BACKING), & BUYGEN & & \\
\hline 1731 & $\boldsymbol{\theta}$ & 90.30 & 72.20 & .05 & -.57 & 1.14 & $60 B$ & बPACITY & ( WH I TE & BACKING ). & BA USCH & - LGMB & \\
\hline 1157 & $\theta$ & 50.35 & 72.95 & .79 &. .30 & 1.48 & $60 B$ & OPACITY & ( WITE & BACKING), & BAUSCH & - LबMB & \\
\hline L262 & $\boldsymbol{\theta}$ & 90.36 & 72.80 & .66 & -.37 & .82 & $60 R$ & OPACITY & ( DHITE & BACKING), & THषING- & ALBERT ( FGRMBRLY & SRL) \\
\hline 1158 & $\boldsymbol{\theta}$ & 90.38 & 73.80 & 1.57 & .04 & 1.29 & $60 D$ & OPACITY & ( WHITE & BACKING), & BNL -2 & & \\
\hline L1C5 & $\theta$ & 90.40 & 74.00 & 1.76 & .10 & 1.25 & $60 \mathrm{~B}$ & OPACI TY & ( \$BITE & BACR ING)。 & HUYGEN & & \\
\hline L308 & ๘ & 90.45 & $73 \cdot 13$ & .99 & -.31 & .97 & $60 \mathrm{~B}$ & OPACITY & ( พнITE & BACKING), & BUYGEN & & \\
\hline L261 & $\boldsymbol{\sigma}$ & 90.47 & 73.20 & 1.07 & -.30 & 1.02 & $60 B$ & OPACITY & ( WHITE & BACKING) & BA USCH & - LеMB & \\
\hline L212 & ฮ & 90.50 & 72.80 & .72 &. .50 & 1.39 & $60 B$ & OPACIIY & ( WHITE & BACKING)。 & BA USCH & - LaMB & \\
\hline L688 & $\theta$ & 90.51 & $73 \cdot 33$ & 1.20 & $\because 28$ & .70 & $60 B$ & GPACITY & ( DBITE & BACKING ), & BA USCE & - LOMB & \\
\hline L.328 & $\theta$ & 90.00 & 72.60 & .58 & -.67 & 1.69 & $60 \mathrm{~B}$ & OPACIIY & ( WHITE & BACE ING ), & BAUSCH & - LबMB & \\
\hline L225 & $\sigma$ & 50.91 & 74.62 & 2.54 & .010 & 1.46 & $60 \mathrm{~B}$ & OPACI IY & ( WBITE & BACE ING). & BAUSCB & - LONB & \\
\hline 2567 & $*$ & 91.02 & 75.25 & 3.16 & .07 & 1.28 & $60 D$ & GPACITY & ( ВHITE & BACKING), & $B N E=2$ & & \\
\hline 1608 & 4 & 91.28 & 76.65 & $4 \cdot 53$ & .43 & .81 & 6OD & EPACI IY & ( & BACKING), & $B N L=2$ & & \\
\hline GMEAN & & 89.75 & $72 \cdot 36$ & & & 1.00 & & & & & & & \\
\hline & & \$5\% & LLIPSE: & 2.75 & .77 & ШIтн & GAMA & $I A=64$ & DEGREES & & & & \\
\hline
\end{tabular}


OPACITY, B\&L TYPE, 89\% BACKING

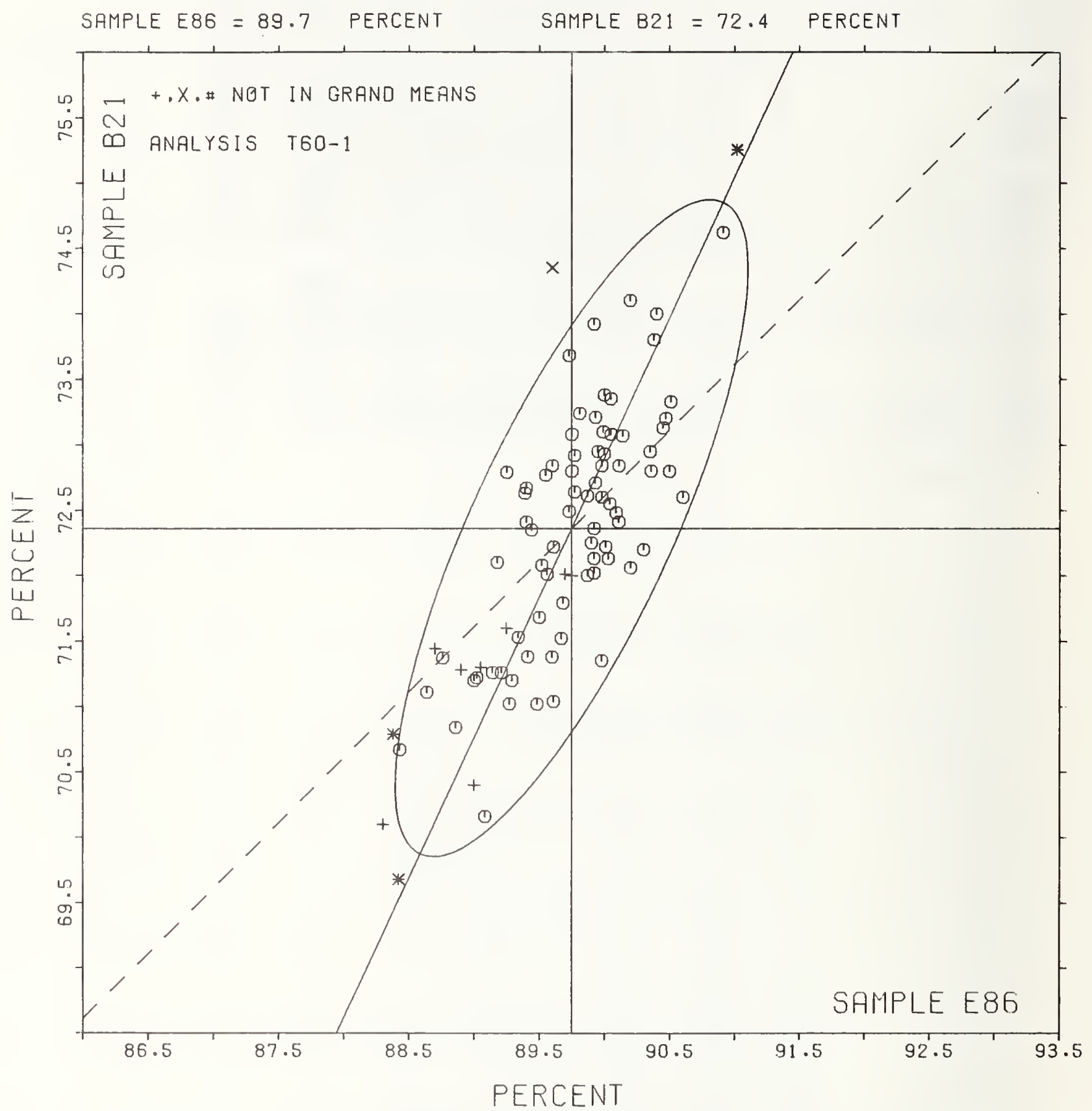


OPACITY (PAPER BACRING) IN PERCENT

TAPPI STANDAFD I425 ES-75, OPACITY GF PAPER (15 DEG./DIFPUSE, ILLUMINANT A) - BSL TYPE

\begin{tabular}{|c|c|c|c|c|c|c|c|c|c|c|c|c|c|}
\hline \multirow{3}{*}{$\begin{array}{l}\mathrm{LAB} \\
\mathrm{CADE}\end{array}$} & \multirow{3}{*}{$\begin{array}{c}\text { SAMPLE } \\
\text { E86 } \\
\text { MEAN }\end{array}$} & \multicolumn{4}{|c|}{ BEND } & \multicolumn{5}{|c|}{ SEMI BLEACAED } & \multirow[t]{2}{*}{ IEST } & $D_{0}=$ & \multirow[t]{2}{*}{10} \\
\hline & & 79 GEA & S PER & SOUARE M & METER & B21 & $65 \mathrm{GR}$ & S PER S & SQUARE $M$. & YETER & & & \\
\hline & & DEV & N. DEV & SDR & $R_{\bullet} S D_{R}$ & MEAN & DEV & N.DEV & SDR & R. SDR & V $\mathbf{A R}$ & $\mathbf{F}$ & $\mathbf{L A B}$ \\
\hline 1118 & 51.40 & -.09 & -.32 & .37 & 1.24 & 75.61 & .00 & .01 & .91 & .83 & $60 \mathrm{C}$ & $\theta$ & L118 \\
\hline $\mathrm{L} 190 \mathrm{C}$ & 91.50 & .01 & .04 & 30 & 1.01 & 75.59 & -.02 & .06 & 1.03 & .93 & $60 \mathrm{C}$ & a & $1190 \mathrm{C}$ \\
\hline L190R & $91 \cdot 83$ & .34 & 1.23 & .24 & .80 & 76.06 & .45 & 1.66 & 1.71 & 1.55 & $60 \mathrm{C}$ & G & L190R \\
\hline$L 236 B$ & 91.43 & -.06 & -.21 & .43 & 1.44 & 75.67 & .06 & .23 & .66 & .60 & $60 \mathrm{C}$ & $\theta$ & L236B \\
\hline L243 & 91.73 & .24 & .87 & .21 & .71 & 75.49 &. .12 & -.43 & .97 & .89 & $60 \mathrm{C}$ & $\boldsymbol{\theta}$ & 1243 \\
\hline 2543 & 51.04 & -.45 & -1.61 & .24 & .80 & 75.22 & -.39 & -1.42 & 1.32 & 1.20 & $60 \mathrm{~V}$ & o & $L 543$ \\
\hline GR. MEAN & $=91.49$ & PE RCENI & & & GRAND MEAN & .75 .61 & PERCENT & & & IEST DETER & INATIE & NS & $=10$ \\
\hline SD MEANS &. .28 & $\begin{array}{r}\text { PE RCEN I } \\
\text { AVER }\end{array}$ & E $\quad S D R$ & .30 & $\begin{array}{l}\text { SD AF MEANS } \\
0 \text { PEBCENT }\end{array}$ & $=.27$ & $\begin{array}{r}\text { PERCENT } \\
\text { AVE }\end{array}$ & SDR & 1.10 & $\begin{array}{r}6 \text { LABS I } \\
\text { PERCENT }\end{array}$ & GRAN L & & ENS \\
\hline
\end{tabular}

TETAL NUMBEE GF LABEFATEEIES FEPGRTING PEBCENT

Best values: E86 91.5 percent

B21 75.5 percent

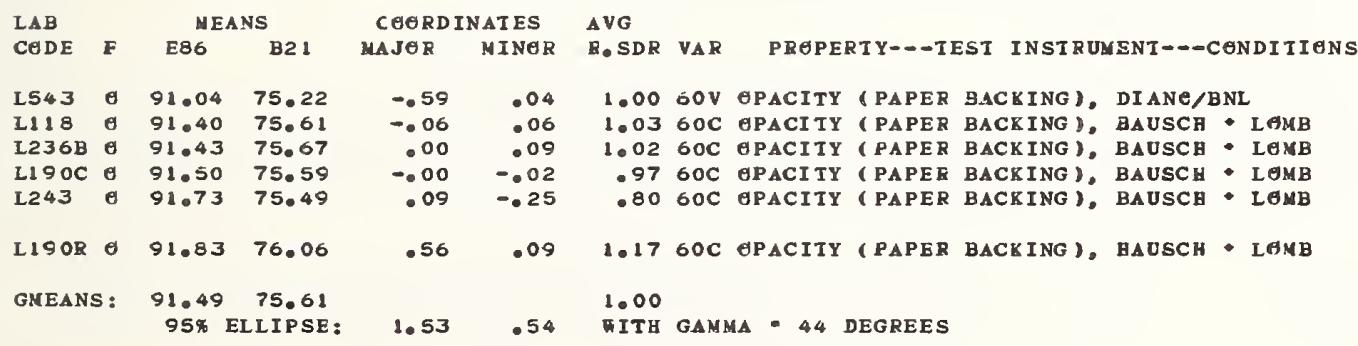


GPACITY (PAPER BACEING) IN PERCENT

TAPPI SUGGESTED METHOD TS19 GS-78, DIFFUSE OPACITY GF PAPER - ILLUNINANT C, ELREPBO IYPE

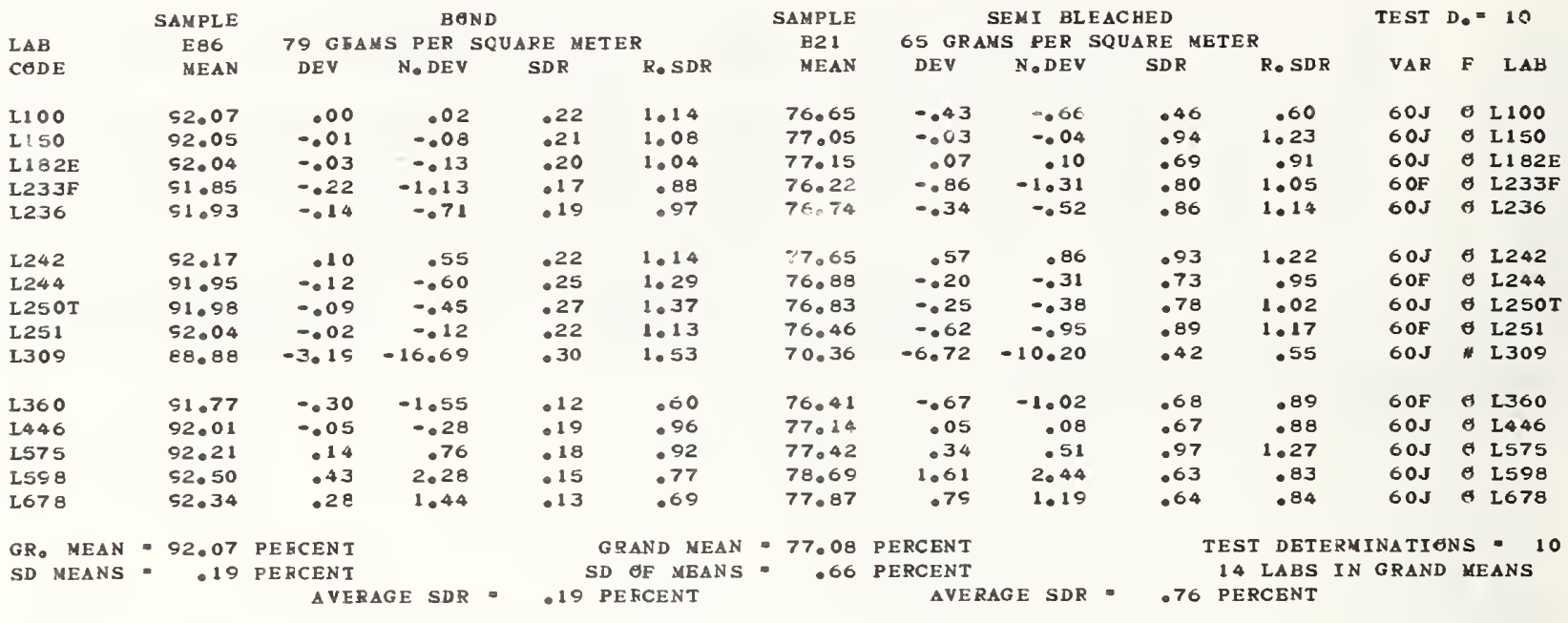

$\begin{array}{lllllllllllll}\text { L626 } & 51.25 & -.82 & -4.27 & .26 & 1.36 & 75.70 & -1.38 & -2.10 & .59 & .77 & 60 & .626\end{array}$ TATAL NUMBER OF LAEGEATEKIES DEPERTING = 16

Best values: E86 $92.1+0.3$ percent

B21 $77.0 \pm 1.0$ percent

The following laboratories were omitted from the

grand means because of extreme test results: 309 .

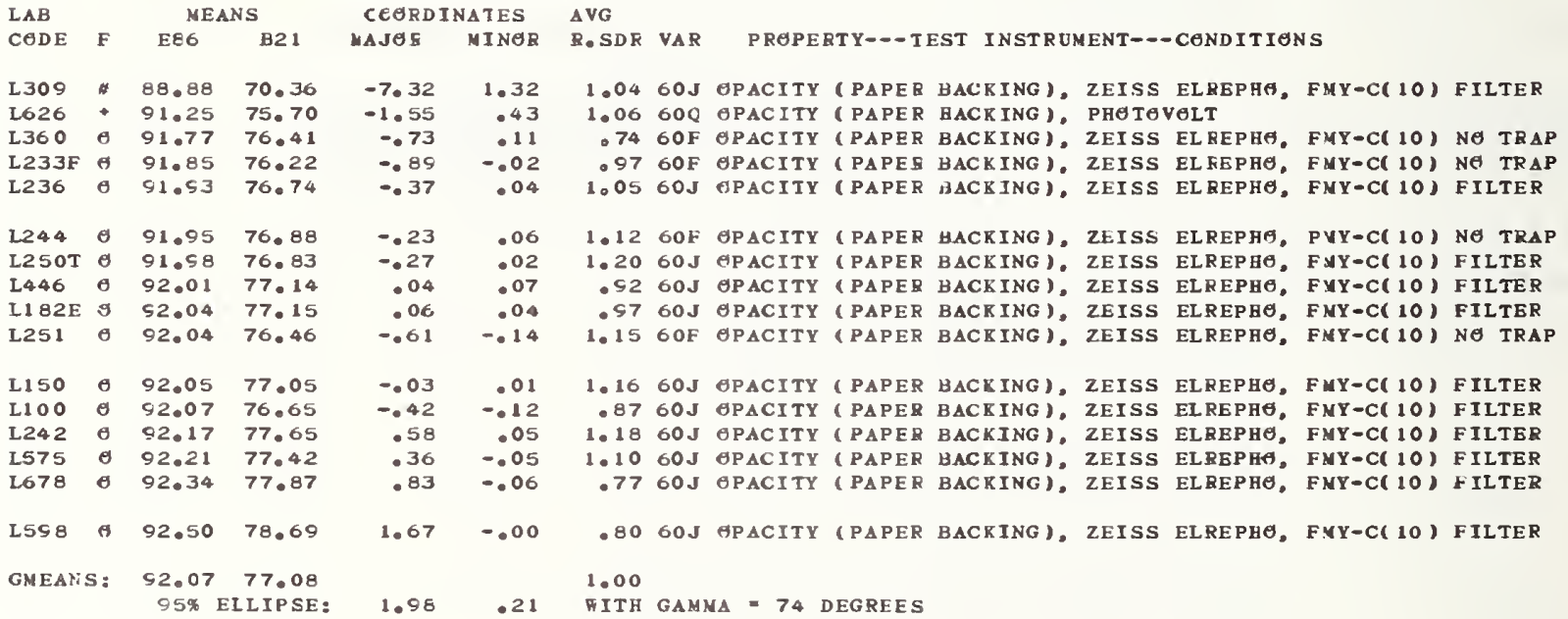




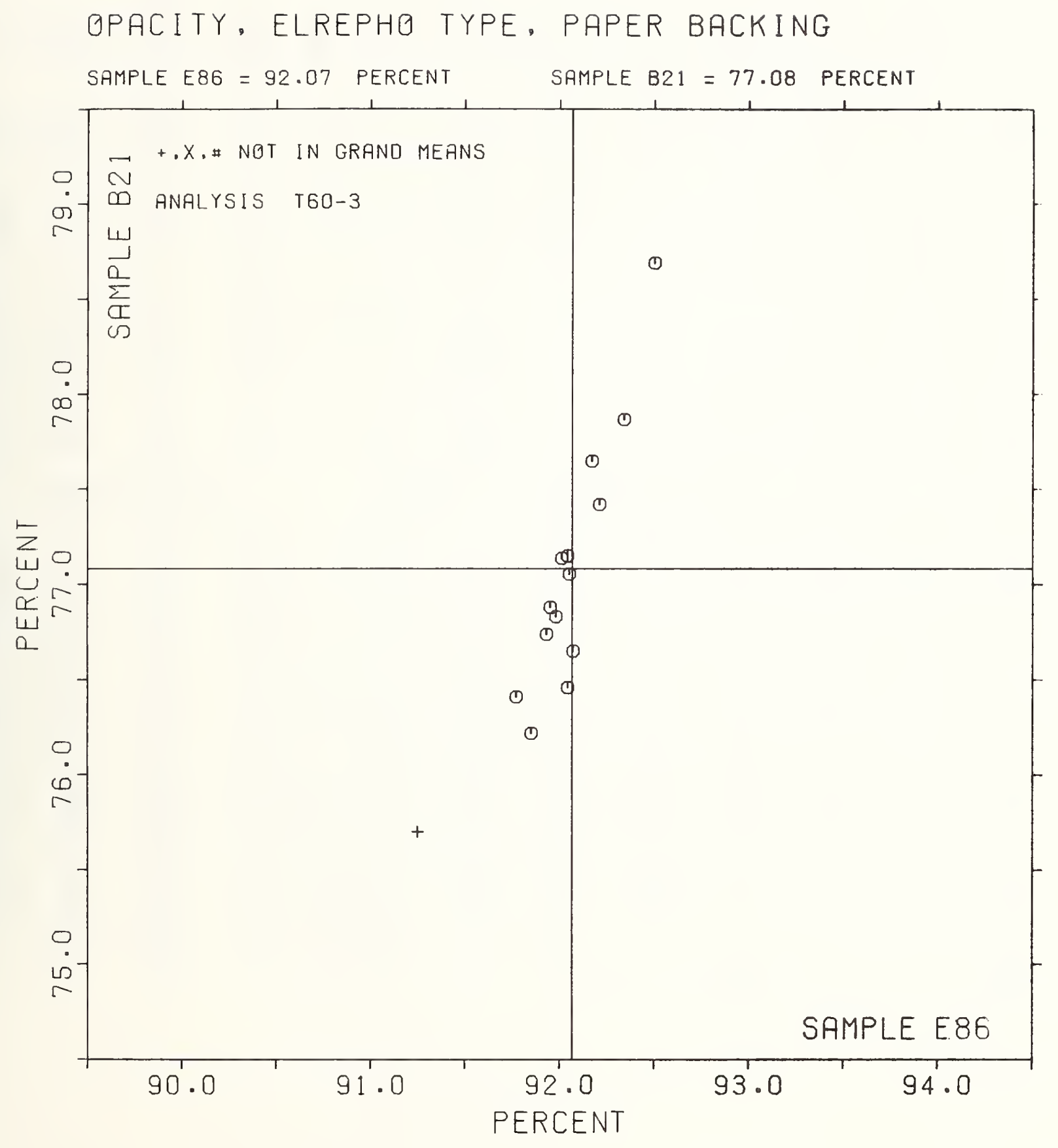


TAPPI STANDARD I452 OS-77, "BRIGHTNESS': MARTIN SWEETS (ACBT \& GE) IS STANDARD FOR THIS ANAIYSIS

\begin{tabular}{|c|c|c|c|c|c|c|c|c|c|c|c|c|c|}
\hline & SANPLE & & PRIN I & IING & & SAMPLE & & PRINT & I I NG & & TEST I & D. - & 8 \\
\hline $\mathbf{L A B}$ & $\mathbf{J 3 6}$ & 94 GLAMS & PEK $S$ & SQUARE ME & ETER & 579 & 73 GRAMS & PER S & SQUARE ME & ETER & & & \\
\hline CEDE & MEAN & DEV N & N. DEV & $S D R$ & $R_{*} S D R$ & MEAN & DEV N & N.DEV & SDR & $R_{\bullet} S D R$ & VAR & $\boldsymbol{F}$ & $\mathbf{L A B}$ \\
\hline 1108 & $83 \cdot 96$ & .03 & .05 & .12 & .96 & 68.59 & .45 & i. 28 & .24 & 1.09 & 654 & $\theta$ & 1108 \\
\hline$x: ? 2$ & 83.40 & .053 &. .88 & .09 & .75 & 68.01 & -.13 & -.36 & .16 & .76 & $65 \mathrm{~N}$ & $\boldsymbol{\theta}$ & 1122 \\
\hline 2132 & 83.45 & -.48 & -.80 & .13 & 1.06 & 68.01 & .13 &. .36 & .26 & 1.20 & $65 N$ & $\theta$ & L 132 \\
\hline 158 & $84 \cdot 10$ & .17 & .28 & .09 & .75 & 68.47 & .34 & .96 & .16 & .73 & $65 N$ & $\theta$ & L158 \\
\hline $1190 \mathrm{C}$ & 83.69 & -.24 & -.40 & .08 & .68 & $68 \cdot 24$ & .10 & .28 & .18 & .85 & $65 A$ & $\theta$ & L $190 \mathrm{C}$ \\
\hline L210 & 84.30 & .37 & .62 & .12 & .97 & 6.8 .21 & .07 & .21 & $\cdot 20$ & .91 & $65 M$ & $\boldsymbol{G}$ & $\mathrm{L} 21 \mathrm{cM}$ \\
\hline L210N & 84.32 & .40 & .66 & .18 & 1.48 & 67.76 & -.38 & -1.07 & .28 & 1.28 & $65 \mathrm{~N}$ & e & $\mathrm{L} 210 \mathrm{~N}$ \\
\hline L211 & 81.64 & -2.29 & $-3 \cdot 82$ & .19 & 1.56 & 68.02 & .11 &. .32 & $\cdot 35$ & 1.63 & $65 N$ & $*$ & 2211 \\
\hline L225 & 83.49 & -.44 &. .74 & .11 & .91 & 68.29 & .15 & .43 & .40 & 1.85 & $65 \mathrm{~N}$ & $\theta$ & L2.25 \\
\hline L243 & 23.31 & -.62 & -1.03 & .17 & 1.40 & 67.80 & .034 & -.97 & .21 & .96 & $65 A$ & $\theta$ & L243 \\
\hline L259 & 83.84 & .05 & .15 & .12 & - 96 & 67.61 &. .53 & -1.50 & .11 & .52 & $65 M$ & $\theta$ & L259 \\
\hline L275 & E3.70 & -.23 & -.38 & .12 & .97 & 68.57 & .44 & 1.25 & .10 & .48 & $65 \mathrm{M}$ & $A$ & L275 \\
\hline L285 & 85.56 & 1.63 & 2.72 & .05 & .42 & 68.17 & .04 & .11 & .17 & .77 & $65 \mathrm{~N}$ & $\star$ & L285 \\
\hline L288 & 83.72 & -.20 & $\because 34$ & .09 & .72 & 67.76 & -.38 & -1.07 & .30 & 1.37 & $65 \mathrm{~N}$ & $M$ & L2 88 \\
\hline L308 & 85.11 & 1.18 & 1.97 & .14 & 1.10 & 68.86 & .72 & 2.07 & .14 & .65 & $65 \mathrm{~N}$ & $\theta$ & L308 \\
\hline L315 & 83.61 & -.32 &. .53 & .06 & .52 & 67.95 & .19 & -.54 & .37 & 1.71 & $65 \mathrm{~N}$ & $\theta$ & L315 \\
\hline 1317 & 83.69 & -.24 & -.40 & .19 & 1.53 & 68.00 & .014 & -.39 & .11 & .49 & $65 M$ & $\theta$ & L317 \\
\hline LS23 & 83.91 & -.02 & .03 & .11 & .91 & 68.32 & .19 & .53 & .23 & 1.04 & $65 \mathrm{~N}$ & $\boldsymbol{\theta}$ & L523 \\
\hline L543 & 83.29 & .064 & -1.07 & .24 & 1.96 & 68.55 & .41 & 1. 18 & .23 & 1.08 & $65 x$ & H & 1543 \\
\hline L565 & 83.56 & -.37 &. .61 & .07 & .60 & 68.27 & .14 & .39 & .07 & .33 & $65 A$ & a & $\mathbf{L 5 6 5}$ \\
\hline L59 8 & Ne DATA & REPORTED & FOR S & SAMPLE J3 & & 67.05 & -.49 & -1.39 & .17 & .78 & $65 *$ & $M$ & L598 \\
\hline 2636 & 84.82 & .90 & 1.49 & .07 & .57 & 67.57 &. .56 & -1.61 & .14 & .64 & $65 \mu$ & 0 & L636 \\
\hline L673R & 83.67 & -.25 & .42 & .15 & 1.21 & 67.85 &. .29 & -.82 & .45 & 2.07 & $65 \mathrm{~N}$ & $\theta$ & L673R \\
\hline 2692 & 80.51 & -3.42 & -5.69 & - 38 & 3.04 & 66.36 & -1.78 & -5.07 & .46 & 2.12 & $65 N$ & $\omega$ & $L 692$ \\
\hline GR. MEAN & - 63.93 & PEACENT & & & GRAND MEAN & - 68.14 & PERCENT & & & TEST DETERM I & INAT T & NS & $\because$ \\
\hline SD MEANS &. $.60 \mathrm{~F}$ & $\begin{array}{l}\text { PERCEN I } \\
\text { AYERAGE }\end{array}$ & E SDR & $.12^{5}$ & $\begin{array}{l}\text { SD OF NEANS } \\
\text { PE RCENT }\end{array}$ & $=.35$ & $\begin{array}{l}\text { PERCENT } \\
\text { AVERAGE }\end{array}$ & E SDR & $\bullet<2$ & $\begin{array}{l}21 \text { IABS IN } \\
\text { PERCENT }\end{array}$ & GRAN D & YE & EANS \\
\hline L105 & 83.65 & -.28 & -.47 & .08 & .61 & 67.49 & -.65 & -1.86 & .11 & .52 & EST & $\bullet$ & L105 \\
\hline L213 & $84 \cdot 25$ & .32 & .53 & .09 & .75 & 67.87 & -.26 & -.75 & .22 & 1.01 & $65 T$ & $\bullet$ & L213 \\
\hline L219 & $85 \cdot 20$ & 1.27 & 2.12 & .13 & 1.06 & 68.81 & .67 & 1.93 & .37 & 1.72 & $65 \mathrm{P}$ & $\bullet$ & L219 \\
\hline L223 & E5. 86 & 1.93 & 3.22 & .07 & .60 & 68.75 & .61 & 1.75 & .13 & .60 & $65 G$ & $\bullet$ & L223 \\
\hline L232 & 85.00 & 1.07 & 1.78 & .00 & .00 & 67.94 &. .20 &. .57 & .18 & .82 & $65 P$ & $\bullet$ & L232 \\
\hline L241 & $84 \cdot 16$ & .23 & .39 & .23 & 1.88 & 68.51 & .37 & 1.07 & .10 & .46 & 651 & $\bullet$ & L241 \\
\hline L249 & 84.51 & .58 & .97 & .11 & .91 & 69.22 & 1.09 & 3.10 & .16 & .73 & $65 P$ & $\bullet$ & 1249 \\
\hline L256 & 83.41 & -.52 & -.86 & .10 & .80 & 67.34 & -.80 & -2.29 & .12 & .55 & $65 H$ & - & L256 \\
\hline L.260 & $84 \cdot 26$ & .33 & .55 & .13 & 1.05 & 09.05 & .91 & 2.60 & .08 & .35 & $65 P$ & $\bullet$ & 1260 \\
\hline L278 & 85.56 & 1.63 & 2.72 & .32 & 2. 59 & 71.15 & 3.01 & 8.60 & .35 & 1.62 & $65 P$ & $\bullet$ & 1278 \\
\hline L301 & 84.05 & .12 & .20 & .14 & 1.15 & 68.32 & .19 & .53 & .13 & .59 & $65 \%$ & $\bullet$ & L301 \\
\hline L312 & 84.87 & .95 & 1.57 & .23 & 1.87 & 70.94 & 2.80 & 7.99 & .18 & .82 & $65 P$ & • & L312 \\
\hline L32 1 & 85.94 & 2.01 & 3.34 & .18 & 1.43 & 70.00 & 1.86 & $5 \cdot 32$ & .00 & .00 & $65 P$ & $\bullet$ & L321 \\
\hline L328 & 87.65 & 3. 72 & 6.20 & - 18 & 1.44 & 71.64 & 3.50 & 9.99 & .23 & 1.07 & $65 P$ & $\bullet$ & L328 \\
\hline 1339 & 86.62 & 2.70 & 4.49 & .35 & 2.86 & 71.87 & 3.74 & 10.67 & $\cdot 23$ & 1.07 & $65 P$ & $\bullet$ & L339 \\
\hline L380 & $84 \cdot 12$ & .20 & $\bullet 33$ & .23 & 1.87 & 72.00 & 3.86 & 11.03 & .00 & .00 & $65 P$ & $\bullet$ & 1380 \\
\hline 1442 & 51.00 & 7.07 & 11.77 & .17 & 1.37 & 72.97 & 4.84 & 13.81 & .24 & 1.12 & $65 T$ & $\bullet$ & 1442 \\
\hline L56 2 & 87.00 & 3.07 & 5.11 & .00 & .00 & $74 \cdot 50$ & 6.36 & 18.17 & .00 & .00 & $65 \mathrm{P}$ & $\bullet$ & L562 \\
\hline L587 & $84 \cdot 37$ & .45 & .74 & .07 & .57 & 68.01 & -.13 & -.36 & .30 & 1.41 & $65 I$ & $\bullet$ & L587 \\
\hline L59 1 & $23 \cdot 85$ & -.08 & -.13 & .08 & .63 & 66.79 & -1.35 & $-3 \cdot 85$ & .09 & .39 & $65 H$ & $\bullet$ & L591 \\
\hline L626 & $86 \cdot 34$ & 2.41 & 4.01 & .19 & 1.56 & 70.24 & 2.10 & 6.00 & .23 & 1.07 & $65 P$ & • & L626 \\
\hline 1684 & 83.77 &. .15 & -.26 & .16 & 1.28 & 66.79 & -1.35 & -3.86 & .83 & 3.84 & $65 \mathrm{H}$ & $\bullet$ & 2684 \\
\hline
\end{tabular}

TETAL NUNBER OF LABERATARIES GEPERTING = 46

Best values: J36 $83.7 \pm 1.2$ percent

$$
\mathrm{J} 7968.0 \pm 0.6 \text { percent }
$$

The following laboratories were omitted from the

grand means because of extreme test results: 211 ,

692. 
TAPPI STANDARD T452 GS-77. "BRIG GTNESS": MARTIN SWEETS (ACBT \& GE) IS STANDARD FOR THIS ANALYSIS

\begin{tabular}{|c|c|c|c|c|c|c|c|c|c|c|c|c|}
\hline LAB & & $\mathbf{M E}$ & NS & CGORD & NATES & AVG & & & & & & \\
\hline CODE & $\mathbf{F}$ & 536 & 579 & MAJEE & MINOF & R. SDR & VAR & PRE & BPERTY $=-$ TEST & INSTRUMENT $-\infty$ & CONDITIONS & \\
\hline L598 8 & M & & 67.65 & & & .78 & $65 \mathrm{M}$ & BLUE & REFLECTANCE & ( DI RECTIGNAL), & MARTIN SWEETS (GE), S-1 & \\
\hline L692 & 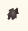 & 80.51 & $66 \cdot 36$ & -3.58 & -1.43 & 2.58 & $65 N$ & BLUE & REFLECTANCE & ( DI RECTIGNAL), & TECHNIDYNE/DIANG/M. $S_{*}$. & $S-4$ \\
\hline L2 11 & * & 81.64 & 68.02 & -2.29 & .12 & 1.60 & $65 N$ & BLUE & REFIECTANCE & (DIRECTI GNAL), & IECBNIDYNE/D IAN $/ \mathrm{N}_{*} \mathrm{~S}_{\bullet}$ & $S-4$ \\
\hline L543 & $\sigma$ & 83.29 & 68.55 &. .60 & .47 & 1.52 & $65 M$ & BLUE & REFLECTANCE & ( DI RECT I GNAL), & MARTIN SWEETS (GE), $S=1$ & \\
\hline 1243 & 6 & $83 \cdot 31$ & 67.80 & -.65 & -.27 & 1.18 & $65 \mathrm{~A}$ & BLUE & REFLECTANCE & ( DIRECTIGNAL). & MARTIN SWEETS ( $\triangle C B T)_{0} S-$ & -2 \\
\hline L1 22 & $\boldsymbol{\sigma}$ & 83.40 & 68.01 &. .54 & -.07 & .75 & $65 \mathrm{~N}$ & BLUE & REF LECT ANCE & (DIRECTIBNAL), & TECHNIDYNE/DIANA/M。S•, & $S=4$ \\
\hline L256 & - & 83.41 & $67 \cdot 34$ & -.59 & -.74 & .68 & $65 H$ & HL UE & REF LECTANCE & ( DIRECTIONAL), & BUNTER & \\
\hline 1132 & 6 & 83.45 & 68.01 & -.49 & -.08 & 1.13 & $65 \mathrm{~N}$ & BLUE & REF LECT A NCE & ( D I RECI IGNAL), & TECANIDYNE/DI ANO/N.S•. & $s=4$ \\
\hline L225 & $\theta$ & 83.49 & $68 \cdot 29$ &. .43 & .19 & 1.38 & $65 N$ & BL UE & REFLECT $\triangle N C E$ & ( DIRECTIENAL), & TE CHNI DYNE/D I ANO / M。 $S_{\bullet}$ 。 & $S-4$ \\
\hline L565 & $\theta$ & 83.56 & 68.27 & -.35 & .17 & .46 & $65 A$ & BLUE & REFLECTANCE & ( DIRECTIGNAL), & MARTIN SWEETS ( $A C B T), S-$ & -2 \\
\hline $\mathbf{L 3 1 5}$ & ब & 83.61 & 67.95 & -.33 & -.16 & 1.11 & $65 \mathrm{~N}$ & BLUE & REF FLECTANCE & (DI RECTIENAL). & TECHNIDYNE/DIANO/M。 $S_{\bullet}$ & $S=4$ \\
\hline L105 & - & 83.65 & 67.49 &.- .34 & -.62 & .57 & $65 \mathrm{~T}$ & BLUE & REFLECTANCE & (DIRECIIGNAL), & HUNTER D25D2N & \\
\hline L673R & a & $83 \cdot 67$ & 67.85 & -.28 & -.26 & 1.64 & $65 \mathrm{~N}$ & BLUE & REFLECT ANCE & (DIRECTIONAL), & TEC HNID YNE/DIANG/No $S_{\bullet}$ & $5-4$ \\
\hline LIS OC & $\boldsymbol{\theta}$ & 83.69 & 68.24 & -.23 & .12 & .76 & $65 A$ & BLUE & REF LECTANCE & (DIRECTIGNAL), & MARTIN SWEETS (ACBT), S- & \\
\hline 1317 & $\theta$ & 83.69 & $68.0 \mathrm{C}$ & -.25 & .11 & 1.01 & $65 \mathrm{M}$ & BLUE & REFLECTANCE & ( DI RECTIGNAL), & MARTIN SWEETS (GE), $S-1$ & \\
\hline L275 & $\boldsymbol{\theta}$ & 83.70 & $68 \cdot 57$ & -.18 & .46 & .72 & $65 \mathrm{M}$ & ELUE & REFLECT ANCE & (DIRECTIENAL), & MARTIN SWEETS (GE), $S=1$ & \\
\hline L28 8 & ๘ & 83.72 & 67.76 & -.24 & -.35 & 1.05 & $65 \mathrm{~N}$ & BLUE & REFLECTANCE & ( DIRECII $\forall N A L)$. & TECBNI DYNE/DIANG/No S* & $5=4$ \\
\hline L684 & - & 83.77 & 66.75 & -.29 & $-1 \cdot 33$ & 2.56 & $65 H$ & BLUE & REFLECT $A N C E$ & (DIRECTIONAL), & HUNTER & \\
\hline L259 & $\boldsymbol{\theta}$ & 83,84 & 67.61 &. .14 &. .51 & .74 & $65 \mathrm{H}$ & BLUE & QEF LECTANCE & ( DIRECTIGNAL). & MARTIN SWEETS (GE), S-I & \\
\hline 1591 & - & 83.85 & 66.79 & -.22 & -1.33 & .51 & $65 H$ & BLUE & REF LECTANCE & ( DIRECTIGNAL). & HUNTER & \\
\hline L523 & a & 83.91 & $68 \cdot 32$ & .00 & .19 & .98 & $65 \mathrm{~N}$ & BLUE & REF LECT ANCE & (DIRECTIGNAL), & TECHNIDYNE/DIANE/M. & $s=4$ \\
\hline L108 & a & 83.96 & 68.59 & .08 & .44 & 1.03 & $65 u$ & BLUE & REFLECT ANCE & (DIRECTIGNAL)。 & MARTIN SWEETS (GE), $S=1$ & \\
\hline L301 & - & 84.05 & 68.32 & .14 & .17 & .87 & $65 G$ & BLUE & REFLECTANCE & ( DIBECTIONAL). & GARDNER & \\
\hline L158 & 6 & $84 \cdot 10$ & 68.47 & .20 & .32 & .74 & $65 \mathrm{~N}$ & BLUE & REFLECTANCE & ( DIRECIIENAL), & TECHNIDYNE/DIANG/M. $S_{0}$ & $S=4$ \\
\hline $\mathrm{L} 380$ & - & 84.12 & 72.00 & $\cdot 58$ & 3.82 & .94 & $65 \mathrm{P}$ & BLUE & REFLECTANCE & ( DI RECTI GNAL). & PHETEVALT & \\
\hline L 241 & - & 84.16 & 68.51 & $\cdot 27$ & . 35 & 1. 17 & 651 & HLUE & REFLECTANCE & (DIRECTIONAL). & HUNTER D25D2A & \\
\hline L213 & - & 84.25 & 67.87 & $\cdot 29$ & -.25 & .88 & $65 \mathrm{~T}$ & BLUE & REFLECTANCE & (DIRECII ANAL), & HUNTER D25D2U & \\
\hline L260 & - & 84.26 & 65.05 & .42 & .87 & .70 & $65 P$ & BLUE & REF LECTANCE & ( DIRECIIONAL), & PHETEVELT & \\
\hline L210M & $\boldsymbol{\theta}$ & $84 \cdot 30$ & $68 \cdot 21$ & - 38 & .04 & .94 & 654 & BLUE & FEFLECTANCE & (DIRECTI ONAL). & MARTIN SWEETS (GE), S=1 & \\
\hline L210N & $\sigma$ & 84.32 & 67.76 & . 36 & -.41 & 1.38 & $65 \mathrm{~N}$ & BLUE & REFLECTANCE & (DIRECTIGNAL), & TECBNIDYNE/DIANO/N. S. & $s-4$ \\
\hline L587 & - & $84 \cdot 37$ & 68.01 & .43 & .17 & .99 & 651 & BLUE & REF LECT A NCE & (DIRECTIGNAL), & HUNTER D25D2A & \\
\hline L249 & - & 84.51 & 69.22 & .69 & 1.02 & .82 & $65 \mathrm{P}$ & BLUE & REFLECT ANCE & (DIRECTIONAL), & PHETOVELT & \\
\hline 1636 & $\boldsymbol{\theta}$ & 84.82 & 67.57 & $\cdot 83$ & -.65 & .61 & $65 \mathrm{M}$ & BLUE & REFLECT ANCE & (DIRECTI GNAL), & MAKIIN SWEETS (GE). & \\
\hline L312 & • & 84.87 & 70.94 & 1.22 & 2.69 & 1.35 & 65P & BLUE & REFLECT ANCE & (DIRECII INAL), & FHOTEVGLT & \\
\hline L232 & 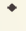 & 85.00 & 67.94 & 1.04 & -.31 & .41 & $65 P$ & BLUE & REFLECTANCE & ( DIRECTIONAL), & F HETEVOLT & \\
\hline 1308 & e & $85 \cdot 11$ & 68.86 & 1.25 & .60 & .87 & $65 \mathrm{~N}$ & BLUE & REFLECTANCE & (DIRECII INAL), & TECHNI DYNE/DIANE/M. $S_{*}$ & $S=4$ \\
\hline L219 & - & 85.20 & 68.81 & 1.33 & .54 & 1.39 & $65 P$ & BLUE & REF LECTANCE & (DIRECTIGNAL), & PHETEVELT & \\
\hline L278 & - & 85.56 & 71.15 & 1.93 & 2.83 & 2.11 & $65 \mathrm{P}$ & HLUE & REF LECT ANCE & (DIRECIIGNAL). & FHETEVELT & \\
\hline L285 & * & 85.56 & 68.17 & 1.63 &. .13 & .60 & $65 \mathrm{~N}$ & BLUE & REFLECTANCE & ( DIRECTIGNAL), & TECHNIDYNE/DIANO/M• $S_{\bullet}$ & $s=4$ \\
\hline L223 & - & e5.86 & 68.75 & 2.98 & .42 & .60 & $65 \mathrm{G}$ & BLUE & REFLECTANCE & (DIRECTIENAI), & GARDNER & \\
\hline L321 & - & 85.94 & $70 \cdot 00$ & 2.18 & 1.65 & .72 & $65 \mathrm{P}$ & BLUE & REFLECT ANCE & (DI RECT IENAL). & PHET EVELT & \\
\hline L626 & - & $86 \cdot 34$ & 70.24 & 2.61 & 1.85 & 1.32 & $65 P$ & BLUE & REFLECTANCE & ( DIRECIIGNAL), & FHбT TVUL I & \\
\hline L339 & - & 86.62 & 71.87 & 3.06 & 3.45 & 1.97 & $65 P$ & BLUE & REFLECTANCE & (DIRECIIGNAL), & PHOIAVELT & \\
\hline L562 & - & 87.00 & 74.50 & 3.69 & 6.02 & .00 & $65 P$ & BLUE & REFLECTANCE & (DIRECTIENAL), & PHETEVOLT & \\
\hline L328 & - & 87.65 & $71 \cdot 64$ & 4.05 & 3.11 & $1 \cdot 26$ & $65 P$ & BLUE & REFLECT ANCE & ( DIRECTIONAI). & PHฮTสVฮLT & \\
\hline 1442 & - & 91.00 & 72.97 & 7.52 & 4.11 & 1.25 & $65 \mathrm{~T}$ & BLUE & REFLECTANCE & (DIRECTIONAL). & HUNTER D25D2M & \\
\hline GME & & $\begin{array}{c}83.53 \\
95 \%\end{array}$ & $\begin{array}{l}\text { 68. } 14 \\
\text { LLI PSE: }\end{array}$ & 1.64 & .94 & $\begin{array}{l}1.00 \\
\text { พITH }\end{array}$ & GAMN & $M A=$ & 5 DEGREES & & & \\
\hline
\end{tabular}


BLUE REFLECTANCE, DIRECTI INAL

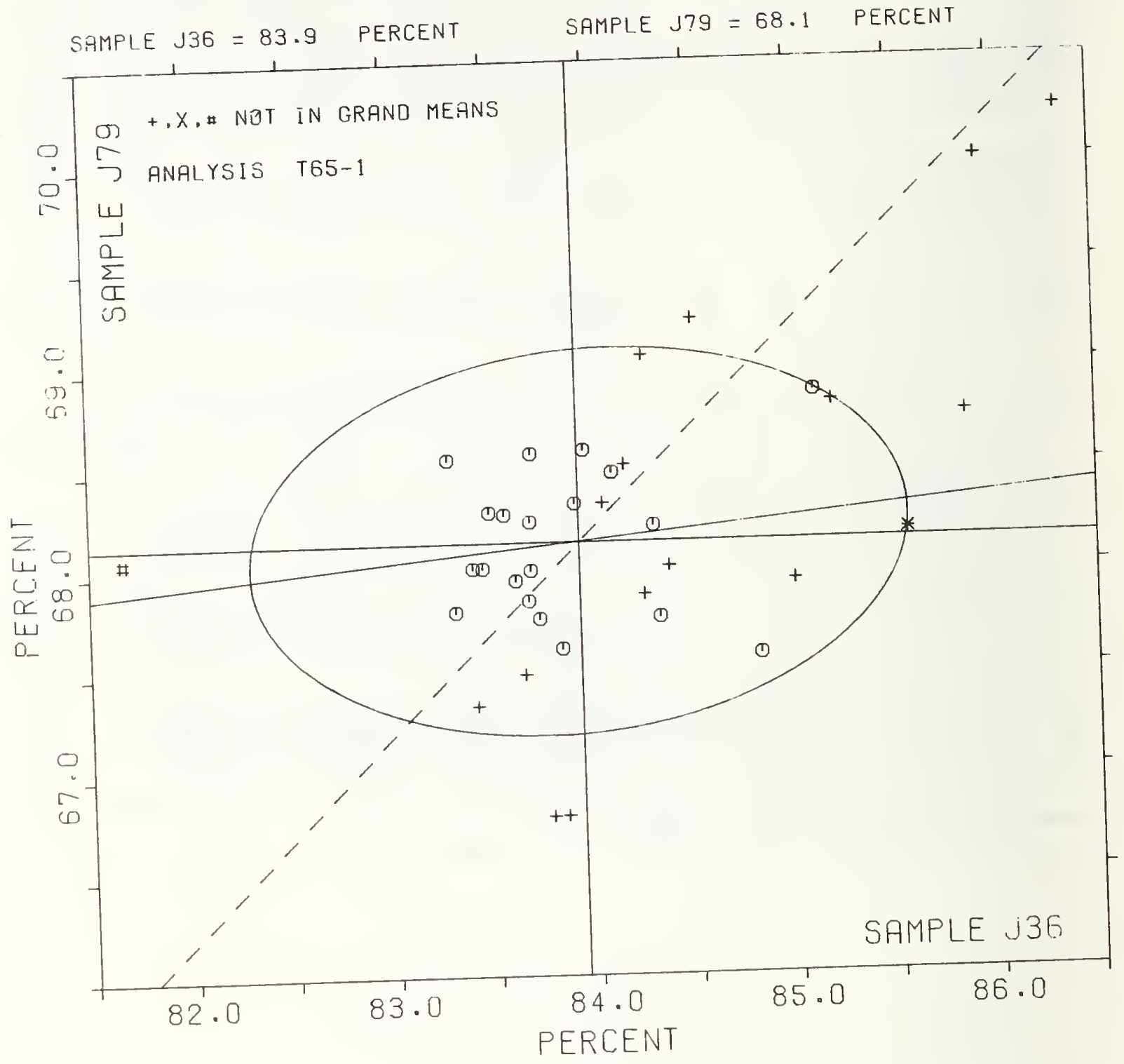


DIFFUSE BLUE REFLECTANCE IN PERCENT (GLESS TRAP)

IAPPI SUGGESTED METHED IS25 SU-72, BRIGETNESS GF PULP (DIFFUSE IIIUMINATIEN AND O DEG. ABSERVATIGN)

\begin{tabular}{|c|c|c|c|c|c|c|c|c|c|c|c|c|c|}
\hline \multirow{3}{*}{$\begin{array}{l}\text { LAB } \\
C \theta D E\end{array}$} & \multirow{3}{*}{$\begin{array}{c}\text { SANPLE } \\
\text { J36 } \\
\text { HEAN }\end{array}$} & \multicolumn{4}{|c|}{ PRINTING } & \multirow{3}{*}{$\begin{array}{c}\text { SAMPIE } \\
\text { J79 } \\
\text { MEAN }\end{array}$} & \multicolumn{4}{|c|}{ PRINTING } & \multirow{2}{*}{ TEST } & \multirow[t]{2}{*}{$D_{*} \cdot$} & \multirow[t]{2}{*}{8} \\
\hline & & 94 G5Al & $S$ PER & SQUARE M] & & & $73 \mathrm{GR}$ & PER & SQUARE $M$ & & & & \\
\hline & & DEV & N. DEV & $S D R$ & R. SDR & & DEV & N. DEV & SDR & $R \cdot S D R$ & VAR & $\mathbf{F}$ & $L A B$ \\
\hline L1CO & 84.62 & .17 & .36 & .09 & 1.10 & 67.47 & -.40 & -.55 & .16 & .92 & $65 F$ & $\theta$ & 2100 \\
\hline 1121 & 84.58 & .13 & .28 & .08 & .89 & 68.74 & .87 & 1.21 & .13 & .73 & $65 K$ & $\boldsymbol{\theta}$ & 2121 \\
\hline$L 136$ & 84.53 & .08 & .17 & .16 & 1.85 & 67.65 & -.22 & -.31 & .17 & .95 & $65 F$ & $\boldsymbol{\theta}$ & 2136 \\
\hline L1 50 & 84.15 & -.30 & -.64 & .06 & .77 & 67.01 &. .87 & -1.20 & .14 & .82 & 650 & $\theta$ & L 150 \\
\hline 1170 & 84.00 & -.45 & -.96 & .00 & .00 & 67.60 &. .27 & .038 & .23 & 1.33 & 658 & $\theta$ & 1170 \\
\hline L210K & 85.05 & .60 & 1.27 & .05 & .61 & 69.28 & 1.41 & 1.95 & .20 & 1.12 & $65 \mathrm{~K}$ & $\boldsymbol{\theta}$ & L2 $10 \mathrm{~K}$ \\
\hline L236 & 84.82 & .37 & .78 & .08 & .90 & $67 \cdot 94$ & .07 & .09 & .09 & .53 & $65 \%$ & $\theta$ & L236 \\
\hline L242 & $84 \cdot 30$ &. .15 & -.31 & .08 & 1.00 & 66.75 & $-1 \cdot 12$ & -1.55 & .32 & 1.82 & $65 F$ & $\boldsymbol{\theta}$ & $\mathrm{I} 242$ \\
\hline L250T & 84.36 & -.10 & -.21 & .13 & 1.55 & 67.66 & -.21 & .29 & .16 & .91 & $65 F$ & $\boldsymbol{\theta}$ & I250T \\
\hline L280 & 84.29 & .016 & -.35 & .09 & 1.06 & 67.72 & .15 & -.21 & .23 & 1.33 & 650 & $\theta$ & 1280 \\
\hline 1325 & 85.08 & .62 & 1.32 & .09 & 1.10 & 67.98 & .10 & .15 & .25 & 1.41 & $65 F$ & $\theta$ & 1325 \\
\hline 2349 & 84.00 & $-.4 \epsilon$ & -.97 & .16 & 1.94 & $67 \cdot 17$ & -.71 & -.98 & .20 & 1.14 & $65 \mathrm{~K}$ & $\theta$ & 2349 \\
\hline 1446 & 84.09 & -.36 &. .77 & .11 & 1.28 & 67.38 &. .49 & -.68 & .15 & .85 & $65 E$ & e & 1446 \\
\hline L573 & 85.53 & 1.08 & 2.29 & .08 & .90 & 68.33 & .46 & .64 & .11 & .65 & $65 F$ & $\theta$ & 1573 \\
\hline L575 & 83.98 & -.48 & -1.01 & .05 & .62 & $67 \cdot 56$ & -.31 &. .43 & .25 & 1.41 & $65 F$ & $\theta$ & 2575 \\
\hline L598 & 84.25 & -.21 & -.44 & .05 & .61 & 69.21 & 1.33 & 1.85 & .09 & .52 & $65 K$ & $\theta$ & 1598 \\
\hline 1636 & 84.83 & $.3 \varepsilon$ & .81 & .05 & .63 & 68.76 & .88 & 1.22 & .16 & .89 & $65 \mathrm{~K}$ & e & 2636 \\
\hline I680 & 83.70 & -.76 & -1.61 & .10 & 1.17 & 67.49 & -.38 & -.53 & .12 & .67 & $65 \mathrm{k}$ & $\boldsymbol{\theta}$ & 2680 \\
\hline
\end{tabular}

GL. MEAN - 84.45 PERCENT

SD MEANS - .47 PERCENT

GRAND MEAN $=67.87$ PERCENT

SD GF MEANS - 72 PERCENT

AVERAGE SDR - .08 PERCENT

AVERAGE SDR -

TEST DETERMINATIONS - 8 18 LABS IN GRAND MEANS

$\begin{array}{llllllllllllll}L 289 & 83.37 & -1.08 & -2.29 & .05 & .55 & 67.34 & -.53 & -.74 & .07 & .42 & 650 & .289\end{array}$ TETAL NUMBEF GF IAEEATERIES EEPERTING = 19

Best values: J36 $84.4+0.7$ percent

J79 $67.8 \pm 1.0$ percent

IAPPI SUGGESTED MEIBOD I525 SU-72, BRIGHTNESS CF PULP (DIFFUSE ILLUMINAIION AND O DEG. OBSERVATION)

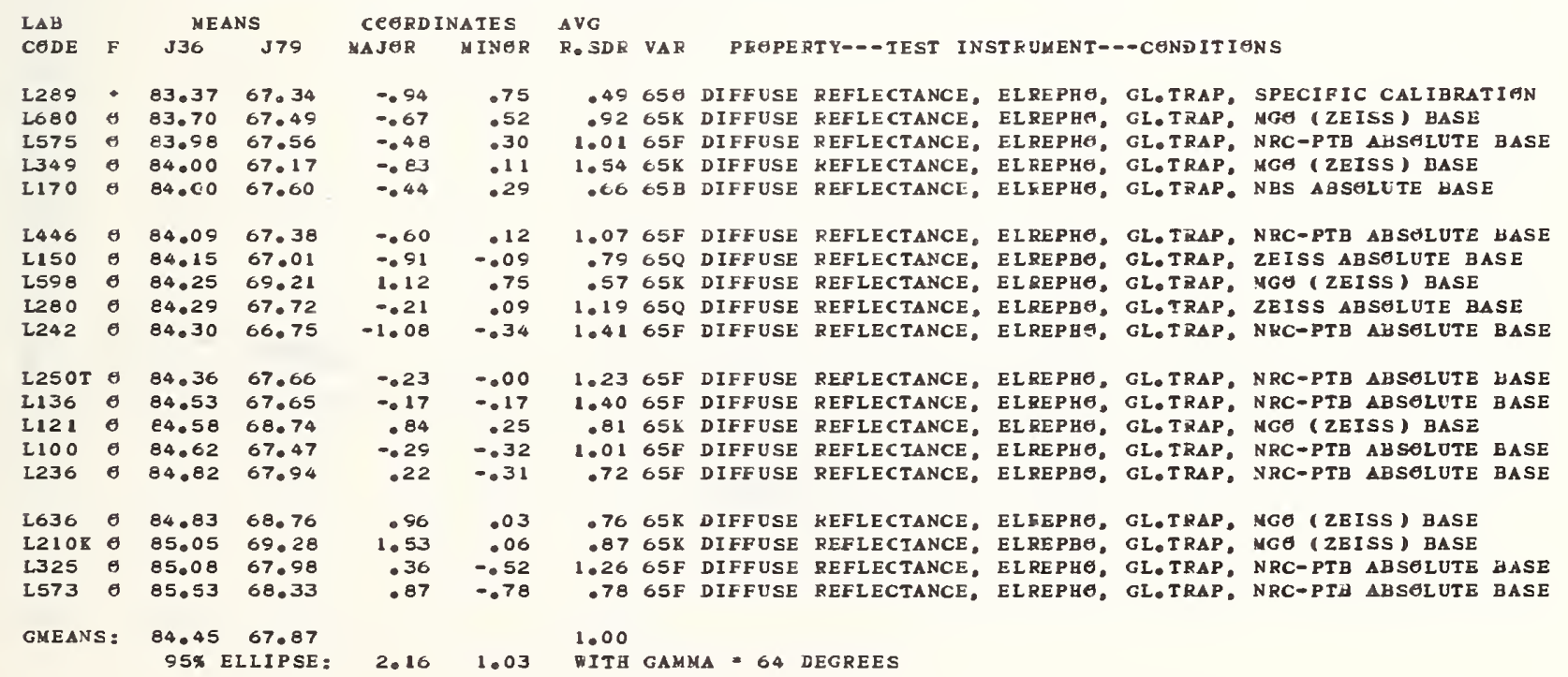


BLUE REFLECTANCE, DIFFUSE, With TRAP

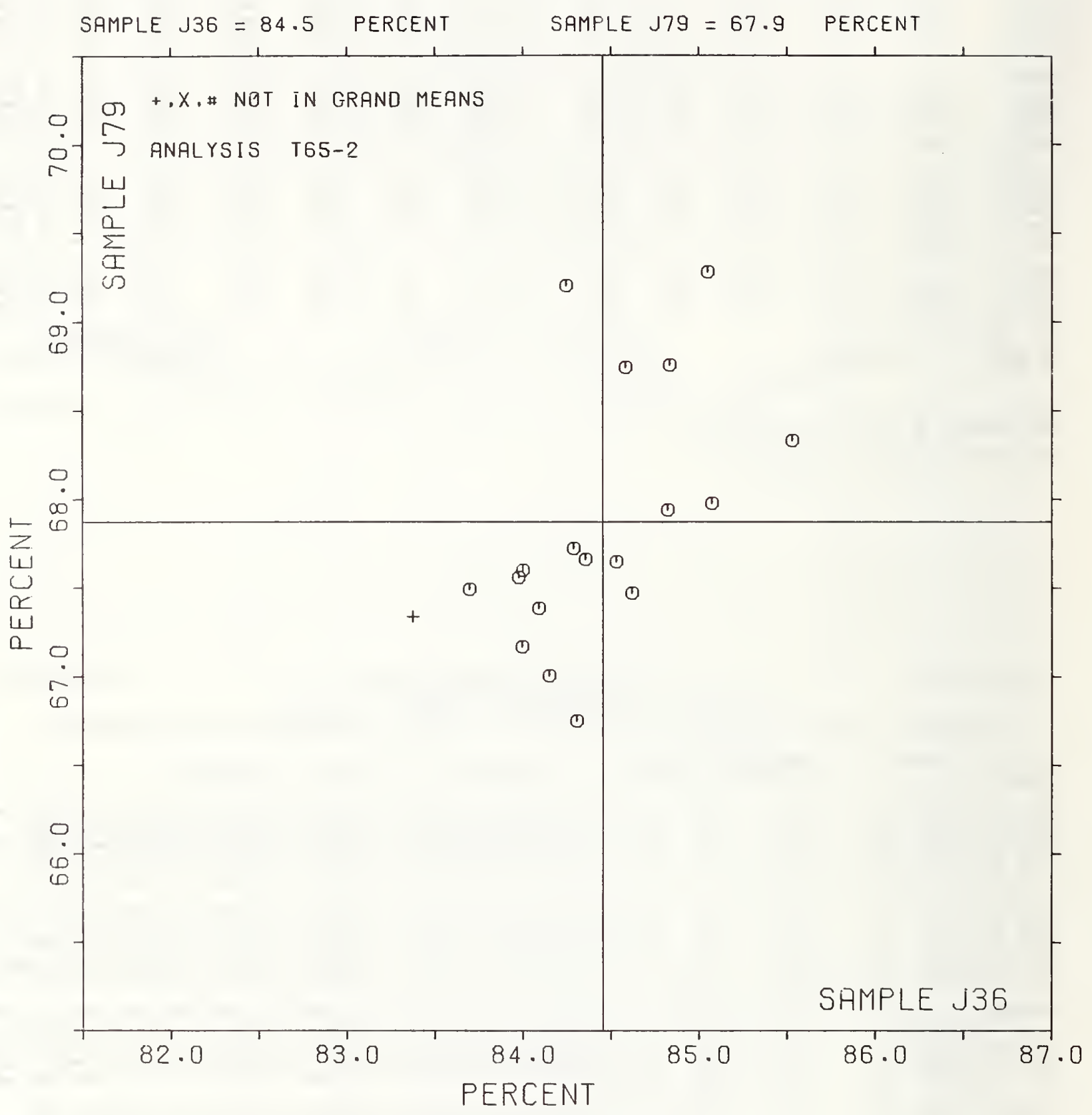


DIFFUSE BLUE REFIECTANCE IN PERCENT (NO GLOSS TRAP)

TAPPI SUGGESTED METHED TS25 SU-72, BRIGBTNESS OF PULP (DIFFUSE ILLUMINATIGN AND O DEG. GBSERVATION)

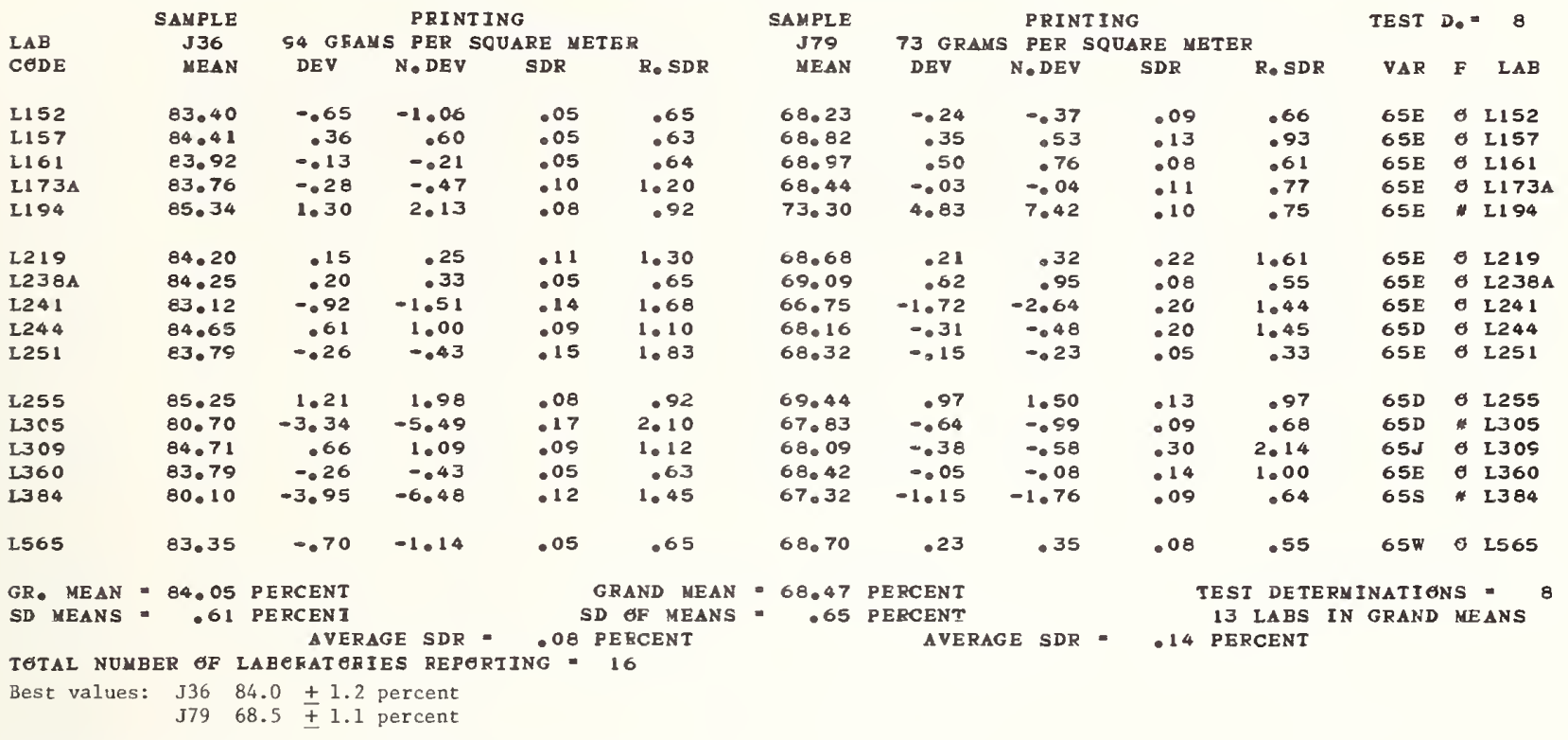

The following laboratories were omitted from the grand means because of extreme test results: 194 , $305,384$.

TAPPI SUGGESTED NETBED TS25 SU-72, BRIGHTNESS GF PULP (DIFFUSE ILLUMINATIGN AND O DEG. GBSERVATIGN)

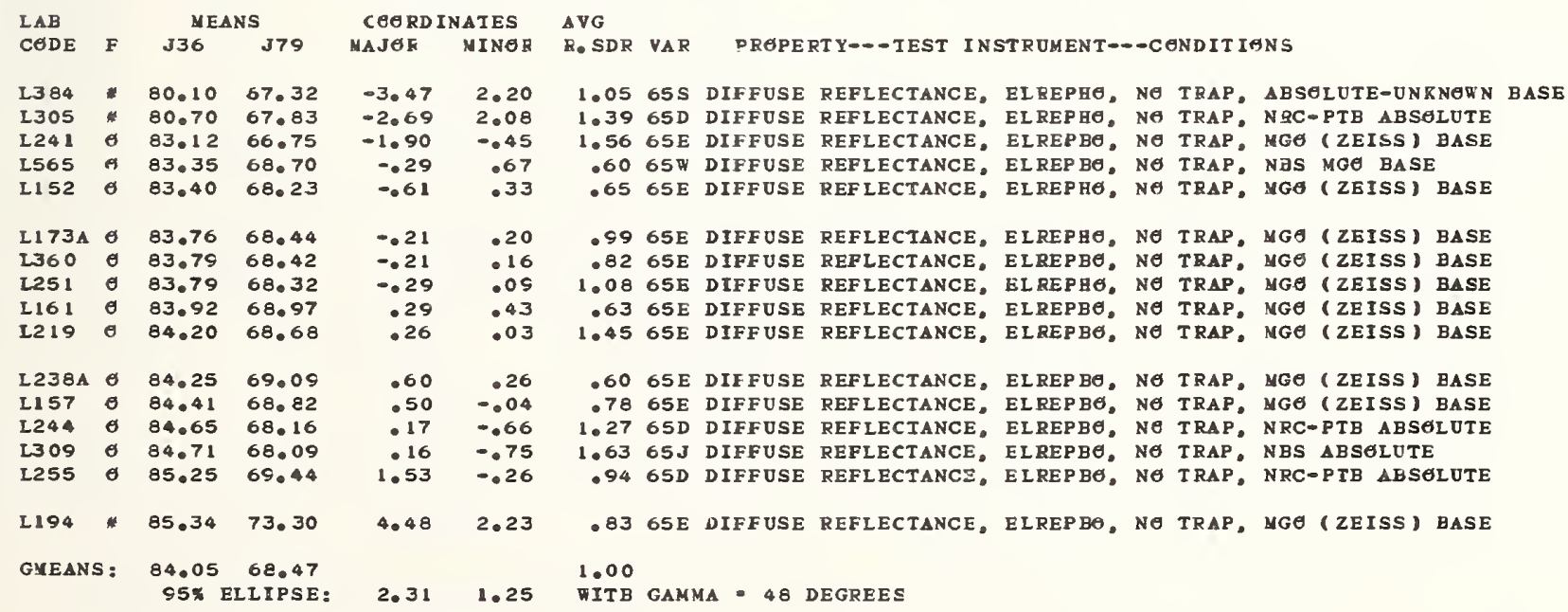




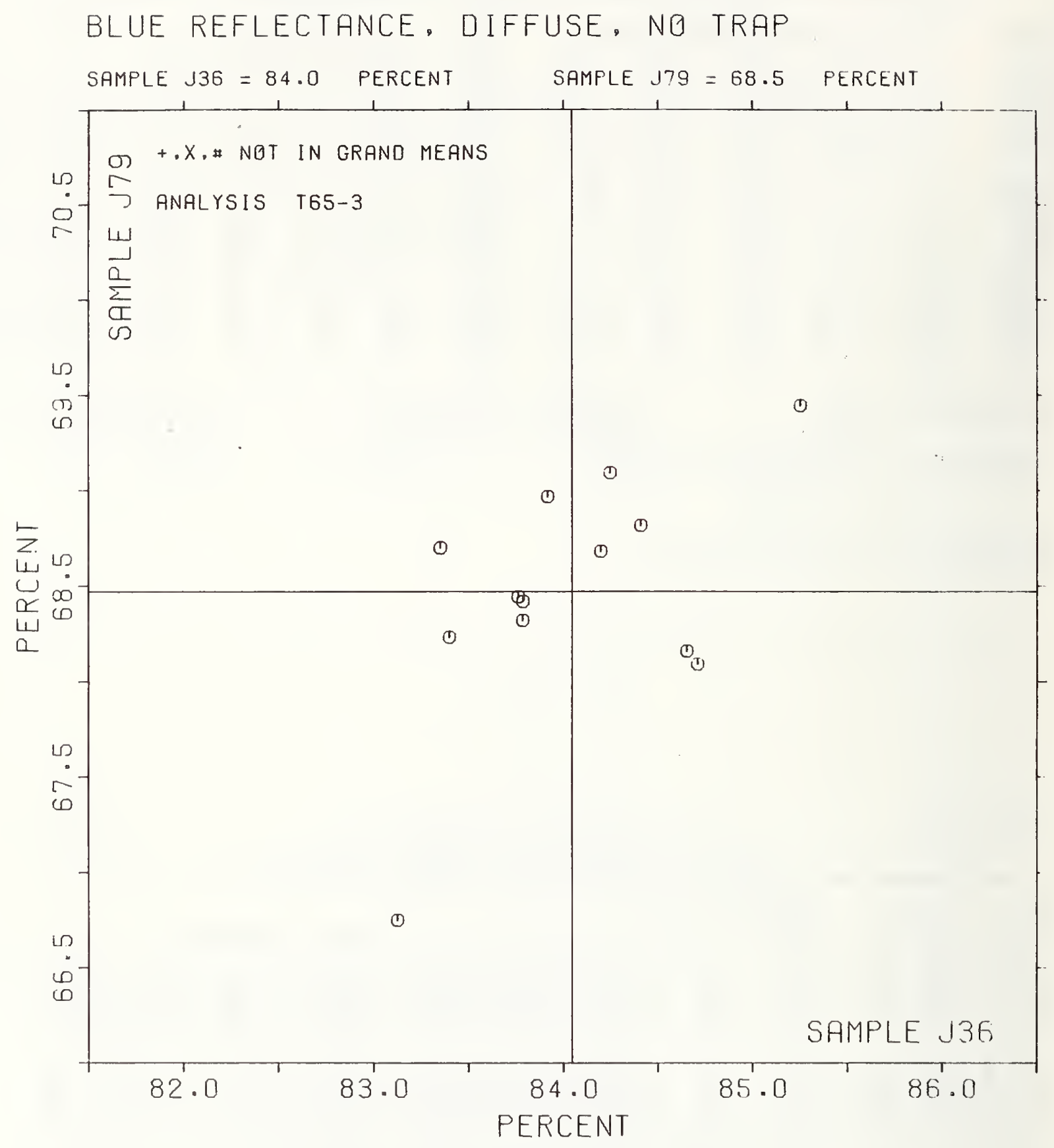


SPECULAR GIESS AT 75 DEGREES, IN GLESS UNITS

TAPPI SIANDARD I480 OS=78, SPECULAR GLOSS OF PAPER AND PAPERBOARD AT 75 DEOREES

\begin{tabular}{|c|c|c|c|c|c|}
\hline \multirow{3}{*}{$\begin{array}{l}\mathrm{LAB} \\
\operatorname{CODE}\end{array}$} & \multirow{2}{*}{$\begin{array}{c}\text { SAMPLE } \\
\text { E87 }\end{array}$} & \multicolumn{4}{|c|}{ COAIED GFFSET } \\
\hline & & $117 G 5$ & PER & SOUARE M & \\
\hline & MEAN & DEV & N. DEV & SDR & R. SDR \\
\hline 2108 & 68.0 & -.3 & -.20 & $2 \cdot 4$ & 1.25 \\
\hline L121 & 69.8 & 1.5 & .86 & 2.9 & 1.50 \\
\hline L122 & 68.5 & - 1 & .08 & 2.7 & 1.40 \\
\hline L1 28 & 66.8 & -1.5 & -.89 & 2.5 & 1.29 \\
\hline L134 & 69.2 & .5 & .51 & 2.0 & 1.06 \\
\hline L136 & 70.5 & 2.2 & 1.27 & 1. 7 & .86 \\
\hline L149 & 61.9 & -6.4 & -3.76 & 2.2 & 1.13 \\
\hline L 153 & 70.4 & $2 \cdot 1$ & 1.21 & 2.0 & 1.04 \\
\hline L 162 & 73.0 & 4.7 & 2.74 & 2.1 & 1.09 \\
\hline L173A & 64.8 & -3.5 & -2.06 & 2.7 & 1.40 \\
\hline L182 & 69.1 & - 2 & .47 & 1.8 & . 95 \\
\hline L1 89 & 68.2 & -01 & .05 & .9 & .46 \\
\hline L1900 & 65.4 & -2.9 & -1.71 & 1.9 & .98 \\
\hline L190R & 67.3 & -1.1 &. .62 & 1.8 & .95 \\
\hline L206 & 68.6 & $\cdot 3$ & .18 & 1.4 & .73 \\
\hline L2 10 & 72.2 & 3. 5 & 2.26 & 1.6 & - 84 \\
\hline L2 11 & 69.5 & $1 \cdot 2$ & .71 & 1.2 & .63 \\
\hline L2 12 & 71.6 & $3 \cdot 3$ & 1.91 & 2.3 & 1.20 \\
\hline L213 & 68.5 & $\cdot 2$ & .11 & 1.6 & .82 \\
\hline L223 & 68.7 & .4 & .22 & 1.5 & .79 \\
\hline L230 & 68.6 & - 3 & .16 & $1 \cdot 3$ & .66 \\
\hline 1243 & 69.8 & 1.5 & .86 & 1.9 & .97 \\
\hline L25 1 & 69.4 & 1.1 & .63 & 1.8 & - 91 \\
\hline L255 & 67.8 & -.5 & -.31 & 1.2 & .64 \\
\hline L256 & $69 \cdot 2$ & - 5 & .52 & 1. 7 & .90 \\
\hline L259 & 67.7 & -.6 & -.37 & $2 \cdot 8$ & 1.47 \\
\hline L262 & 68.9 & .6 & - 33 & 2.5 & 1.29 \\
\hline L278 & 62.9 & -5.4 & -3.18 & $2 \cdot 8$ & 1.45 \\
\hline L291 & 66.6 & -1.7 & -1.02 & 3.1 & 1.59 \\
\hline L30 1 & 67.4 & -1.0 & -.57 & 2.4 & 1.27 \\
\hline L315 & 67.9 & $=4$ & -.25 & $2 \cdot 6$ & 1.35 \\
\hline L3 17 & 68.2 & -.1 & .009 & 2.2 & 1. 14 \\
\hline L321 & 69.4 & 1.1 & .63 & 2.0 & 1.06 \\
\hline L323 & $66 \cdot 9$ & -1.4 & -.82 & $2 \cdot 7$ & $1 \cdot 38$ \\
\hline 1.328 & 67.2 & -1.1 & -.65 & 1.4 & .70 \\
\hline 1339 & 65.4 & -2.5 & -1.71 & 3.2 & 1.64 \\
\hline 1349 & 65.8 & -2.5 & -1.46 & 1.3 & .70 \\
\hline L.37 2 & 67.7 &. .6 &. .34 & 1.6 & .84 \\
\hline 2388 & 60.3 & -8.0 & -4.70 & 1.1 & . 55 \\
\hline L396 & 69.3 & 1.0 & .57 & 1.0 & .51 \\
\hline$L 456$ & 68.7 & $\cdot 3$ & .19 & 1.6 & .84 \\
\hline$L 483$ & 68.0 & -.3 & -.20 & 1.7 & .86 \\
\hline L573 & 65.4 & $-2 \cdot 9$ & -1.71 & $2 \cdot 2$ & 1.15 \\
\hline L574 & 66.9 & $-1 \cdot 5$ & -.85 & 1.7 & .88 \\
\hline L583 & 69.5 & 1.1 & .06 & 2.0 & 1.02 \\
\hline L587 & 69.6 & 1.3 & .74 & $\cdot 8$ & .44 \\
\hline L592 & 68.6 & .3 & .18 & 1.5 & .76 \\
\hline L598 & 67.8 &. .5 & -.29 & 1.2 & .62 \\
\hline 2643 & $68 \cdot 3$ & -.0 &. .03 & 1.7 & .87 \\
\hline 1668 & 68.4 & .0 & .02 & 2.5 & 1.32 \\
\hline 1670 & 65.2 & -3.1 & -1.83 & 2,8 & 1.43 \\
\hline L688 & 68.4 & .0 & .02 & 1.2 & .63 \\
\hline
\end{tabular}

CGATED BGOK
SAMIE
E5 118 GRAMS PER SQUARE MBTER

MEAN DEV N.DEV SDR R,SDR

$\begin{array}{rrrrr}69.3 & 1.2 & .75 & .6 & .56 \\ 70.0 & 1.9 & 1.20 & 1.3 & 1.17 \\ 67.3 & -.8 & -.54 & .8 & .74 \\ 68.7 & .6 & .38 & .9 & .87 \\ 69.5 & 1.4 & .89 & 1.3 & 1.16\end{array}$

TEST $D_{0}=10$

71.2

3.11 .99

62.9

70.7

65.6

$-5.2$

2.6

$4.3 \quad 2.74$

$-2.5-1.60$

67.7

66.

$68 \cdot 1$

$\begin{array}{rr}-0.4 & -.26 \\ -3.0 & -1.92 \\ -1.5 & -.97\end{array}$

$\begin{array}{ll}-.0 & -.03 \\ -.5 & -.029\end{array}$

69.4

68.8

69.4

67.1

1.3
.7
1.3

1.7

$-1.0$

.83
.43
.82

66.7

69.3

67.4

69.

-1.4
1.2
-.07

$\because 7$

1.4

.50
.76

68.8

70.8

61.8

.7
2.7

.05

67.1

2.7
-6.3

$-6.3$

.04
1.75
-4.00
.062

68.5

67.1

67.9

70.1

68.5

66.2

-1.0
-.2
2.0

2.0

$-1.4$

66.6
67.9

67.2

59.9

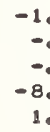

67.3

67.9
66.4

66.7

69.1

$\begin{array}{rr}-.8 & -.53 \\ -.2 & -.15 \\ -1.7 & -1.09 \\ -1.4 & -.08\end{array}$

69.4

67.1

66.9

65.7
66.0

1.3

$\begin{array}{ll}-1.0 & -.62 \\ -1.2 & -.78\end{array}$

$-2.5 \quad-1.57$

$-2.2 \quad-1.37$

66.6

$-1.5 \quad-.95$

GRAND MEAN $=68.1$ GLESS DNITS

SD OP MEANS = 1.6 GLOSS UNITS

REAN - 68,3 GLESS UNITS

AVERAGE SDR *
AVERAGE SDR =

$\begin{array}{rr}.6 & .58 \\ 1.2 & 1.09 \\ 1.7 & .62 \\ 1.0 & .92 \\ 1.3 & 1.23 \\ 1.0 & .96 \\ 1.7 & 1.55 \\ 1.7 & 1.56 \\ 1.5 & 1.37 \\ 1.3 & 1.14\end{array}$

$1.3 \quad 1.14$

$1.4 \quad 1.31$

$\begin{array}{rr}1.9 & .79 \\ 1.0 & .88\end{array}$

$.8 \quad .70$

0

1.

1.04

$\begin{array}{rl}.8 & .78 \\ 1.0 & .92\end{array}$

1.61 .48

$\begin{array}{rr}1.9 & .83 \\ 1.7 & 1.55\end{array}$

$\begin{array}{ll}1.7 & 1.55 \\ 2.0 & 1.85\end{array}$

.9 .79

$\begin{array}{rr}1.0 & .91 \\ 1.1 & 1.02 \\ 1.1 & 1.01\end{array}$

$.7 \quad .67$

.7
$1.1 \quad 1.04$

$2.0 \quad 1.83$

1.1 .96

$\begin{array}{ll}1.1 & .96 \\ 1.1 & .97\end{array}$

$\begin{array}{rr}1.0 & .91 \\ 1.0 & .89\end{array}$

$1.0 \quad 89$

$\begin{array}{ll}1.0 & .95 \\ 1.3 & 1.19\end{array}$

$1.0 \quad .88$

$1.0 . .93$

.6 .53

$\begin{array}{lr}1.0 & .88 \\ 1.4 & 1.27 \\ 1.1 & 1.05\end{array}$

$\begin{array}{rr}1.1 & 1.05 \\ .9 & .78 \\ 1.1 & 1.05\end{array}$

$\begin{array}{rr}.8 & .70 \\ 1.1 & .97\end{array}$
VAR F LAB

$75 H \circlearrowleft 2108$

$75 \mathrm{H}$ L L 121

$75 \mathrm{H} \quad \theta \quad \mathrm{L} 122$

$75 G$ ค L 128

$75 \mathrm{H}$ o I134

$75 G \quad 0 \quad L_{136}$

$75 G$ * L 149

$75 \mathrm{G}$ o L 153

$75 G$ L162

$75 G$ o L173A

$75 H \quad \theta \quad L 182$

$75 P$ O L189

$75 \mathrm{G}$ o L $190 \mathrm{C}$

$75 \mathrm{G}$ o L L $190 \mathrm{R}$

$75 H$ H L206

$75 \mathrm{H}$ o L210

$75 \mathrm{H} O \mathrm{I} 211$

$75 \mathrm{P}$ ○ 212

$75 H$ o 1213

75 H O L 223

75H $0 \quad$ L230

758 o L243

$75 \mathrm{G}$ o $\mathrm{L} 251$

$75 G \quad \sigma \quad$ L255

$\begin{array}{lll}75 H & \theta & \text { L } 256\end{array}$

75H L259

$75 \mathrm{~K}$ o L L262

$75 \mathrm{G}$ L2 278

$\begin{array}{llll}75 \mathrm{~K} & \boldsymbol{\sigma} & \mathrm{L} 291\end{array}$

$75 \mathrm{H}$ o $\mathrm{L} 301$

$75 \mathrm{G}$ e L 315

$75 \mathrm{~F}$ o $\mathrm{L} 317$

$75 \mathrm{G}$ O L321

$75 H \quad O \quad L 323$

$75 \mathrm{~F} O \mathrm{~L} 328$

$75 P$ O 1339

75H o L345

75日 O L372

$75 \mathrm{P}$ L 388

$75 G$ o 1396

$75 \mathrm{H}$ o L456

$75 \mathrm{H}$ o L483

$75 G \quad$ o $L 573$

$75 G$ o L574

$75 \mathrm{H}$ म 2583

$75 H$ ค $L 587$

75H $\theta$ L592

75H $\boldsymbol{G}$ L598

$75 \mathrm{H}$ o L643

$75 G$ a L668

$75 H$ o 1670

$75 G$ G L 688

TEST DETERMINATI 49 LABS IN GRAND MEANS 1. 1 GLOSS UNITS

$\begin{array}{lllllllllllll}L 250 & 63.4 & -4.5 & -2.88 & 1.3 & .70 & 59.5 & -8.6 & -5.50 & 1.2 & 1.08 & 750 & .250\end{array}$

TETAL NUMBER OF LABGRATEIES EEPGRTING

Best values: $\mathrm{E} 8768+3$ gloss units

$$
\text { E5I } 68 \pm 2 \text { gloss units }
$$

The following laboratories were omitted from the

grand means because of extreme test results: 149,

278,388 . 
SPECULAR GLESS AT 75 DEGREES, IN GLOSS UNITS

IAPPI SIANDARD I480 OS-78, SPECULAR GLOSS OF PAPER AND PAPERBOARD AT 75 DEGREES

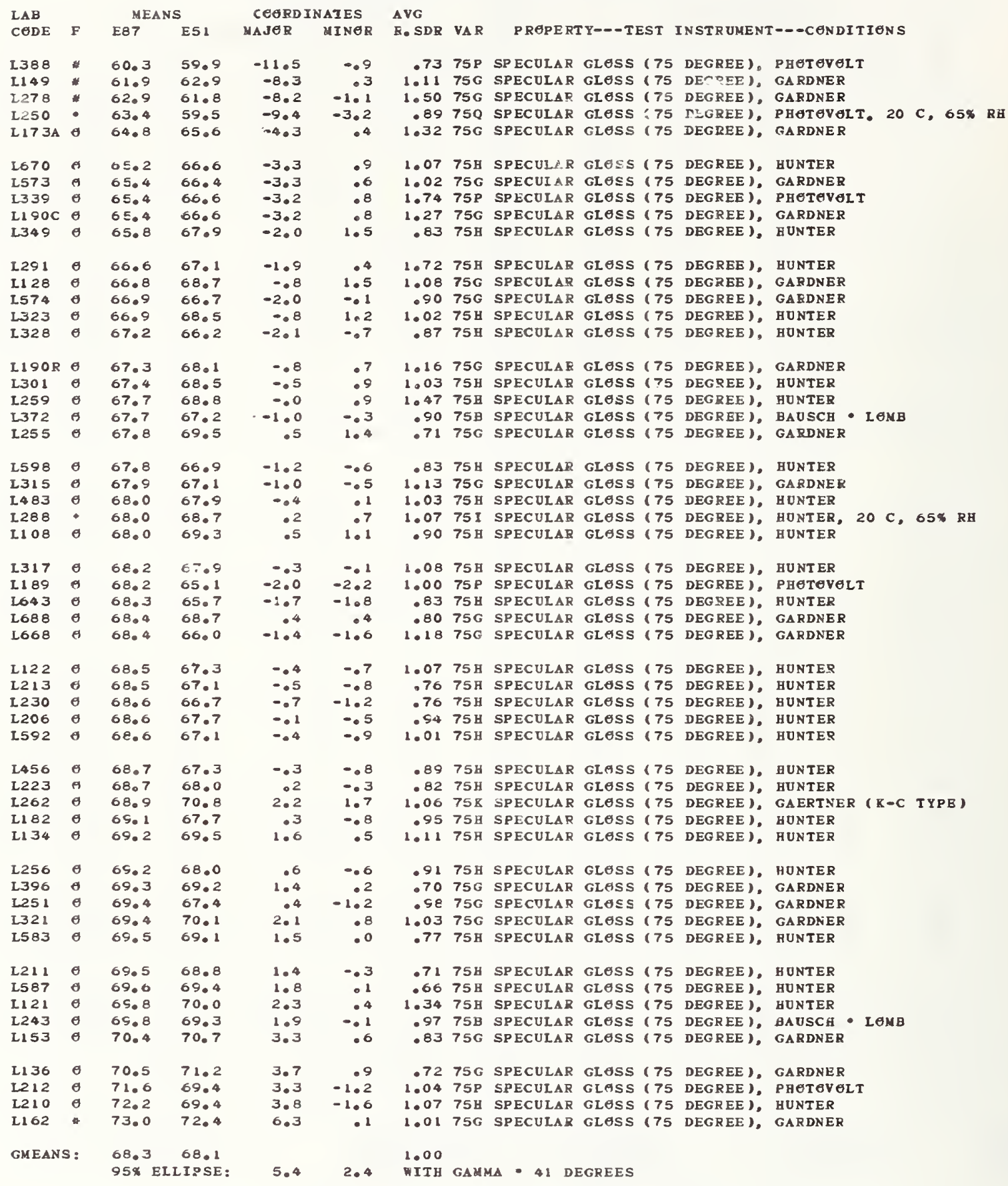


SPECULAR GLOSS, 75 DEGREE

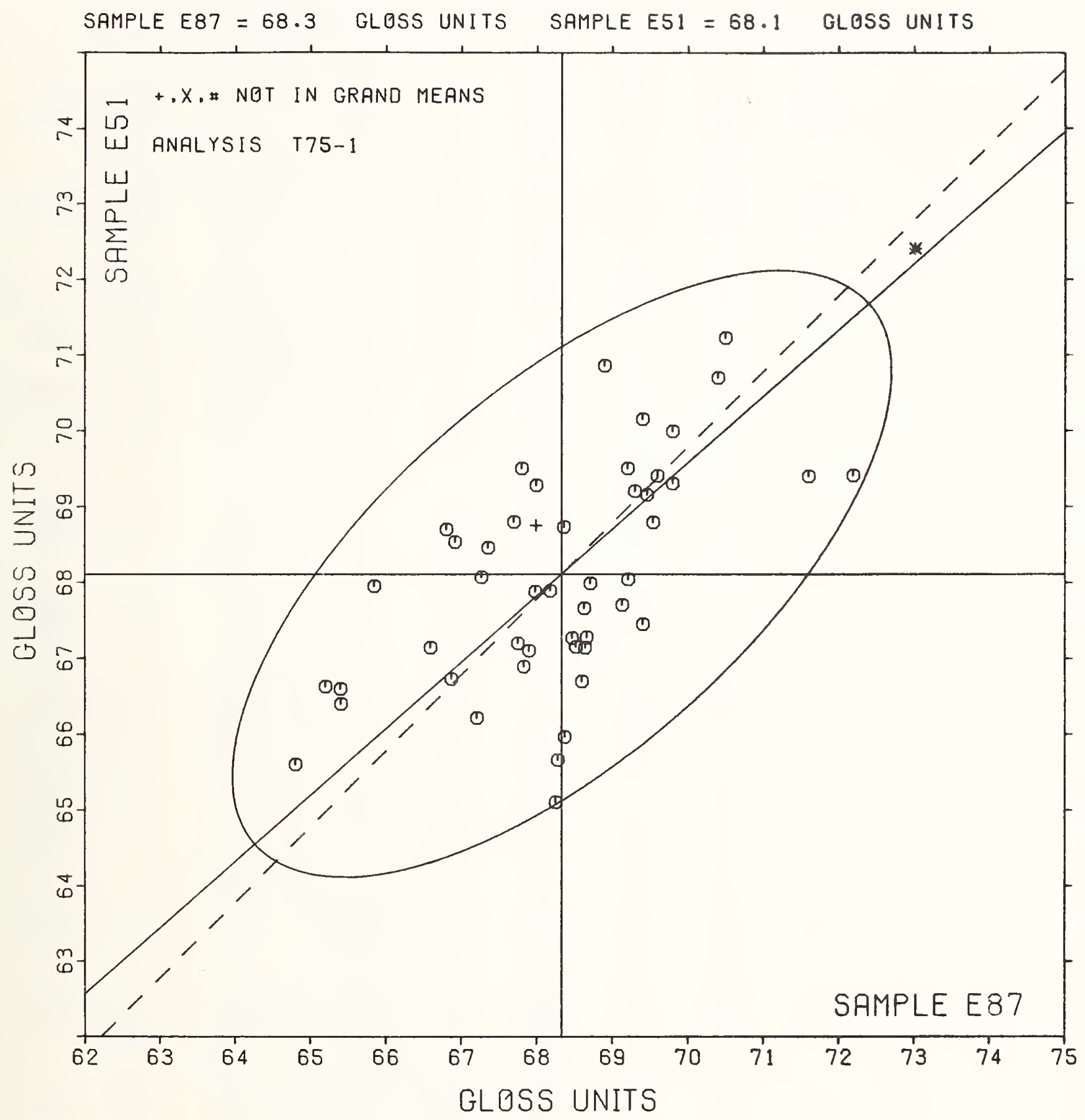

41 
TAPPI STANDARD T411 OS-76

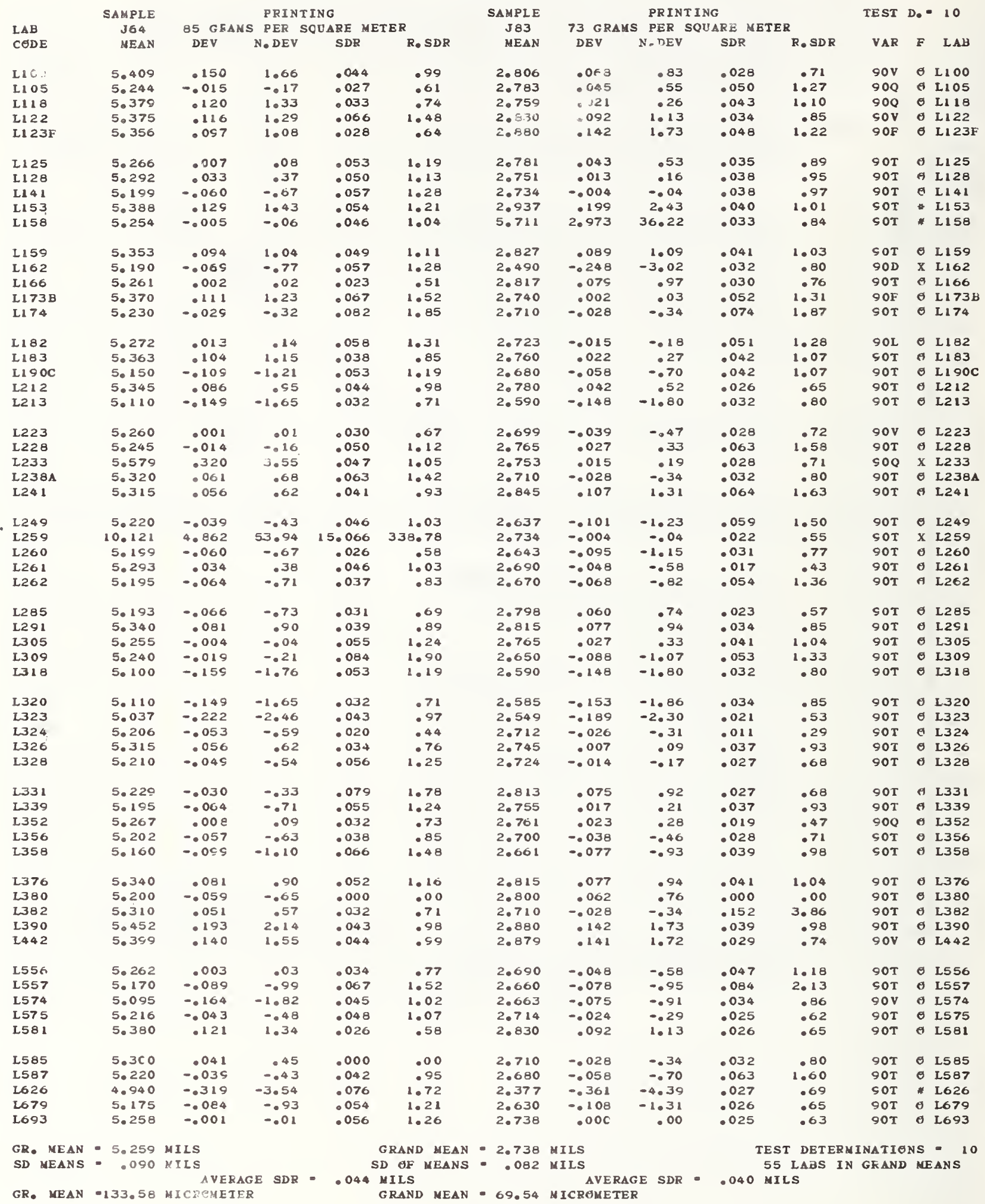




$$
\text { ANALYSIS I90-1 TABLE } 1
$$

THICRNESS (CALIPER), THOUSANDTHS GF AN INCB

TAPPI STANDARD T411 GS=76

IAB

$\operatorname{CODE}$

SAMPLE

J64

PRINIING

O5 GEAMS PER SQUARE METER

DEV

N. DEV

SDR

5.090

5.190

5.303

.165
.065

.065
.044

$-1.87$

I 185

I2426

1243

5. 162

1.63

1.03
-1.08

.057
.025

.025

.041

.026

SAMPIE
J83

J83

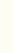

DEV

2.860

2.698

2.742

2.825

2.672

5.236

$-.023$

5.280

.021

.25

.034
.063

139614

L563

5.340

5.041500 .63

.789
.052

.047

.78
1.42

17.74

1.16

1.06

5.135

.007

.08

.048

.039

$5.211 \quad-.048$
$.043 \quad .020$

1.08
.87
.44

TOTAL NUMBER OF LABEFATERIES BEPERTING $73^{\bullet}$

.87

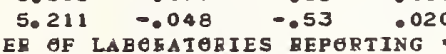

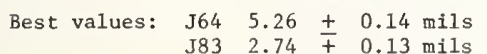

The following laboratories were omitted from the

grand means because of extreme test results: 158 ,

626. 
THICENESS (CALIPER), THEUSANDTHS OF AN INCH

TAPPI STANDARD T41I ES-76

\begin{tabular}{|c|c|c|c|c|c|c|c|c|c|c|c|c|c|}
\hline $\mathbf{L} \mathbf{A B}$ & & MEA & & $\operatorname{ce\theta RD} 1$ & VAIES & AVG & & & & & & & \\
\hline CODE & $\boldsymbol{F}$ & $\sqrt{64}$ & J83 & MAJER & $M I N \theta R$ & R.SDR & VAR & PROPERT & YO- TEST IN & STRUMENT - - & $--\operatorname{coNDI}$ & ITIENS & \\
\hline 1626 & $\#$ & 4.940 & 2.377 & -.478 & .058 & 1.20 & $90 \mathrm{~T}$ & TH IC ENESS & ( CAI IPER), & $T M I$ 。 & MeTER & DRI VEN & \\
\hline L323 & $\theta$ & 5.037 & 2.549 & -.291 & .006 & .75 & $90 \mathrm{~T}$ & THICBNESS & ( CALIPER), & T노. & METER & DRI VEN & \\
\hline L106 & - & 5.090 & 2.860 &. .045 & .204 & 1.52 & $90 \mathrm{C}$ & THICENESS & ( $C A L I P B R)$, & CADY. & BAND & DRIVEN & \\
\hline$\therefore 54$ & $\theta$ & 5.095 & 2.663 &. .172 & .053 & .94 & $90 \mathrm{~V}$ & THICENESS & $\left(\mathrm{CA}^{\top} \mathrm{IPE}\right)$, & TMI, & MOTOR & DRI VEN, DIGITIZED & \\
\hline L318 & a & 5.100 & 2.590 & -.217 & .005 & .99 & $90 \mathrm{~T}$ & TRICRNES S & ( CAI IPER), & IMI, & MOTOR & DRI VEN & \\
\hline L320 & $\theta$ & 5.110 & 2. 585 & -.213 & -.015 & .78 & $90 \mathrm{~T}$ & THIC I N N & (,$\triangle$ LIPER ), & TMI, & MOTOR & DRI VEN & \\
\hline L213 & $\theta$ & 5.110 & 2.590 & -.205 & .011 & .76 & $90 \mathrm{~T}$ & THICDNESS & ( CALIPER), & TMI. & MeTER & DRI VEN & \\
\hline L576 & $\bullet$ & 5.135 & 2.662 &. .143 & .026 & 1.08 & $90 \mathrm{C}$ & THICRNESS & ( CAIIPER), & CADY, & BAND & DR I VEN & \\
\hline L190C & $\theta$ & 5.150 & 2.680 & -.120 & .029 & 1.13 & $90 \mathrm{~T}$ & THICRNESS & ( CALIPER), & TMI, & MOTOS & DR I VEN & \\
\hline L358 & $\theta$ & 5.160 & 2.661 & .0125 & .008 & 1.23 & $90 \mathrm{I}$ & THICKNESS & (CALIPER), & TM I, & METER & DRI VEN & \\
\hline L 243 & 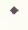 & $5 \cdot 162$ & 2.672 & .0116 & .015 & .65 & $90 \mathrm{~s}$ & THICENESS & ( CALIPER), & SCHGPPER, & HAND & DRI VEN & \\
\hline L557 & $\theta$ & 5.170 & 2.660 & .0118 & .001 & 1.83 & $90 T$ & THICKNESS & ( CALIPER). & TMI & METER & DRIVEN & \\
\hline 1679 & $\theta$ & 5.175 & 2.630 & .0134 &. .025 & .93 & $90 \mathrm{~T}$ & THICERESS & ( CALIPER), & TNI, & MeTER & DRI VEN & \\
\hline L1 85 & - & 5.190 & 2.698 & -.078 & .016 & .54 & $90 \mathrm{~B}$ & THICENESS & ( CAL IPER), & $A M T H O R$, & BAN D & DRIVEN & \\
\hline L 162 & $\mathrm{x}$ & 5.190 & 2.490 & -.216 & .139 & 1.04 & $90 \mathrm{D}$ & TH ICRNESS & (CALIPER), & CADY, & METER & DRI VEN & \\
\hline L285 & ค & 5.193 & 2.798 & .009 & .089 & .63 & $90 \mathrm{~T}$ & THICRNESS & ( CALIPER), & TM I, & METER & DRIVEN & \\
\hline 1339 & $\theta$ & 5.195 & 2.755 & -.036 & .055 & 1.09 & 901 & THICKNESS & ( CAIIPER), & TMI, & MeTeR & URIVEN & \\
\hline L262 & $\theta$ & 5.195 & 2.670 & -.093 & .008 & 1.09 & $90 \mathrm{I}$ & THICENESS & ( CALIPER)。 & THI, & METEF & DRI VEN & \\
\hline L 141 & $\theta$ & 5.199 & 2.734 & .0047 & .037 & 1.13 & $90 \mathrm{~T}$ & THICENESS & ( CALIPER), & IMI, & METER & DRI VEN & \\
\hline L 260 & $\theta$ & $5 \cdot 199$ & 2.643 &. .108 & .0031 & .68 & SOT & THICKNESS & ( CAIIPER), & TMI, & METOR & DRI VEN & \\
\hline 5380 & $\theta$ & 5.200 & 2.800 & -.003 & .086 & .00 & $90 \mathrm{~T}$ & T H I CE NESS & ( CALIPER), & TMI, & MOTOR & DRI VEN & \\
\hline L356 & $\theta$ & 5.202 & 2.700 & -.067 & .010 & .78 & $90 \mathrm{~T}$ & THICKNESS & ( CAL IPER $)$, & TMI, & MOTER & DRI VEN & \\
\hline $\mathbf{L 3 2 4}$ & $\theta$ & 5.206 & 2.712 & -.057 & .016 & .36 & 901 & THIC KNESS & ( CALIPER), & TMI, & METER & DRI VEN & \\
\hline L328 & $\theta$ & 5.210 & 2.724 & -.046 & .022 & .96 & $90 \mathrm{~T}$ & THICKNESS & $(\mathrm{CALIPER})$, & TMI, & MOTOR & DR I VEN & \\
\hline L684 & + & 5.211 & 2.830 & .025 & .101 & .67 & $90 \mathrm{U}$ & THICKNESS & ( C $\triangle L I P E R)$, & TMI, & HAN D & DRIVEN & \\
\hline L575 & 9 & 5.216 & 2.714 & -.048 & .011 & .85 & $90 \mathrm{~T}$ & THICRNESS & ( CALIPER), & TMI, & MOTER & DRI VEN & \\
\hline L249 & $\theta$ & 5.220 & $2 \cdot 637$ & $-.0 \leqq 6$ & .0 .049 & 1.27 & $90 \mathrm{~T}$ & THICKNESS & $(C \triangle L I P E R)$, & TMI, & METOR & DII VEN & \\
\hline 1587 & $\theta$ & $5 \cdot 220$ & 2.680 & .0067 & .017 & 1.27 & $90 \mathrm{~T}$ & THICRNESS & ( CAIIPER), & TN I, & MET TOR & DRI VEN & \\
\hline L331 & $\theta$ & $5 \cdot 229$ & 2.813 & .028 & .076 & 1.23 & $90 \mathrm{~T}$ & ТВICKNESS & $(C A I I P E R)$, & TMI, & METAR & DRI VEN & \\
\hline 2174 & $\theta$ & 5.230 & 2.710 & -.040 & $\because C 01$ & 1.86 & SOT & ТНICKNESS & ( CAIIPER), & TM I, & MOTOR & DRI VEN & \\
\hline 1251 & 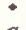 & 5.236 & 2.726 & .024 & .007 & .78 & $90 \mathrm{H}$ & THICKNESS & ( CAL IPER), & L $\cdots$, & METER & $20 \mathrm{c}, 65 \%$ & $\mathbf{R} \mathbf{F}$ \\
\hline L309 & $\theta$ & 5.240 & 2.650 & -.072 & .053 & 1.62 & SOT & IHICRNESS & ( CALIPER), & TMI, & METOR & DRI VEN & \\
\hline 1105 & $\theta$ & 5.244 & 2.783 & .019 & .044 & .94 & 900 & THICENESS & ( CAL IPER ), & EMVECO, & MOTER & DRIVEN & \\
\hline L228 & $\theta$ & 5.245 & 2.765 & .008 & .030 & 1.35 & $90 \mathrm{~T}$ & THICENESS & ( CAIIPER), & TM I, & MOTER & DRI VEN & \\
\hline 1158 & \# & 5.254 & 5.711 & 1.970 & 2.227 & $\cdot 94$ & $90 T$ & TEICRNESS & ( CALIPER), & TMI, & MOTOF & DRIVEN & \\
\hline 1305 & $\theta$ & 5.255 & $2 \cdot 765$ & .015 & .023 & 1.14 & $90 \mathrm{~T}$ & THICKNESS & ( CAIIPER), & TMI, & MATEE & DRI VEN & \\
\hline 1693 & $\theta$ & 5.258 & 2.732 &. .000 & .001 & .95 & $90 \mathrm{~T}$ & ЈHICENESS & ( CALI PER), & TMI, & MOTAR & DR I VEN & \\
\hline L 223 & $\theta$ & 5.260 & 2.699 & -.025 & -.030 & .70 & $90 \mathrm{~V}$ & THICKNESS & ( CALIPER), & TMI, & METER & DRI VEN, DIGITIZED & \\
\hline L1 66 & $\theta$ & $5 \cdot 261$ & 2.817 & .054 & .058 & .64 & sol & TEICENESS & ( CAIIPER), & TMI, & METER & DRI VEN & \\
\hline L556 & $\theta$ & 5.262 & 2.650 &. .029 & -.038 & - 98 & $90 \mathrm{~T}$ & THICKNESS & ( CALIPER), & TMI, & METER & DRI VEN & \\
\hline L567 & - & $5 \cdot 266$ & 2.674 & -.037 &. .052 & 1.19 & SOB & THICRNESS & ( CALIPER), & $\triangle M T H O R$, & HAND & DR IVEN & \\
\hline 1125 & $\theta$ & 5.266 & 2.781 & .034 & .028 & 1.04 & $90 \mathrm{~T}$ & THICKNESS & ( CAIIPER), & TM I, & METEF & DRI VEN & \\
\hline $\mathbf{L 3 5 2}$ & $\theta$ & $5 \cdot 267$ & 2.761 & .022 & .012 & .60 & 900 & THICKNESS & ( CALIPER), & ENVECO, & METOR & DRI VEN & \\
\hline 1182 & $\theta$ & 5.272 & 2.723 & .000 &. .020 & 1.30 & $90 \mathrm{~L}$ & THICKNESS & ( CALIPER), & $\mathrm{L} \cdot \mathrm{w}$, & METER & DRI VEN & \\
\hline $\mathbf{L} 344$ & $\bullet$ & 5.280 & 2.690 & -.016 & .050 & 1.11 & 900 & THICENESS & ( CAL IPER ), & TMI & HAND & DR I VEN & \\
\hline L1 28 & $\theta$ & 5.292 & 2.751 & .034 & .012 & 1.04 & SOT & THICKNESS & ( CALIPER), & TMI, & MeTEF & DRI VEN & \\
\hline L261 & $\theta$ & $5 \cdot 293$ & 2.690 & -.006 & .058 & .73 & $90 \mathrm{~T}$ & THICKNESS & ( CALIPER). & IMI, & METER & DRI VEN & \\
\hline L585 & $\theta$ & 5.300 & 2.710 & .012 & .048 & .40 & $90 \mathrm{~T}$ & THICRNESS & ( CAIIPER), & TMI & MeTEK & DR I VEN & \\
\hline L $242 \theta$ & - & 5.303 & 2.742 & .036 & -.026 & .58 & 900 & T H ICKNESS & ( CAI IPER). & MESSMER, & METAR & DRIVEN, & \\
\hline L382 & $\theta$ & 5.310 & 2.710 & .020 & .055 & 2.28 & $90 \mathrm{~T}$ & THICKNESS & ( CALIPER), & TMI & METER & DRI VEN & \\
\hline 1326 & $\theta$ & 5.315 & 2.745 & .047 & -.032 & .85 & $90 \mathrm{~T}$ & THICENESS & ( CAIIPER), & TMI, & MOTOR & DRI VEN & \\
\hline 1241 & $\theta$ & 5.315 & 2.845 & .113 & .043 & 1.28 & $90 \mathrm{I}$ & THICKNESS & ( $C A \perp I P E R)$, & INI, & MOTOR & DRIVEN & \\
\hline L238A & $\theta$ & 5.320 & 2.710 & .027 &. .061 & 1.11 & $90 \mathrm{~T}$ & THICKNESS & ( C $\triangle L I P E R)$, & TMI, & MOTEE & DRI YEN & \\
\hline 1616 & - & $5 \cdot 336$ & 2.832 & .120 & .019 & .96 & $90 \mathrm{C}$ & THICRNESS & ( CALIPER), & CADY, & H AN D & DRI VEN & \\
\hline 1563 & + & 5.340 & 2.820 & .115 & .008 & 1.38 & $90 \mathrm{U}$ & ТВICENESS & ( CALIPER), & TMI, & HAND & DR I VEN & \\
\hline 1291 & $\theta$ & 5.340 & 2.815 & .112 & .004 & .87 & $90 \mathrm{~T}$ & THICKNESS & ( CAIIPER), & TMI. & MOTER & DRI VEN & \\
\hline L376 & $\theta$ & 5.340 & 2.815 & .112 & .004 & 1.10 & $90 \mathrm{~T}$ & THICENESS & ( CALIPER), & TMI, & MOTOR & DR I VEN & \\
\hline L21 2 & $\theta$ & $5 \cdot 345$ & 2.780 & .092 &. .025 & .82 & SOI & THICENESS & ( CALIPER), & TMI, & YOTOR & DRI VEN & \\
\hline 1159 & G & 5.353 & 2.827 & .130 & .004 & 1.07 & $90 \mathrm{~T}$ & THICKNESS & ( CALIPER), & TMI, & MOTER & DRI VEN & \\
\hline L123F & $\theta$ & 5.356 & 2.880 & .167 & .042 & .93 & $90 F$ & THICENESS & ( CAI IPER), & FEDERAL & MOTEE & DRIVEN & \\
\hline 1183 & $\theta$ & 5.363 & 2.760 & .093 &. .052 & - 56 & $90 \mathrm{~T}$ & THICENESS & ( CA LIPER), & TMI, & METER & DR I VEN & \\
\hline L1738 & $\theta$ & 5.370 & 2.740 & .085 & .072 & 1.41 & $90 \mathrm{~F}$ & TH ICBNESS & ( CAL I PER), & FE DERA L & MET बR & DRI VEN & \\
\hline 1122 & $\theta$ & 5.375 & 2.830 & .148 & -.008 & 1.17 & $90 \mathrm{~V}$ & TH IC KNESS & ( CAIIPER), & I M I, & MOTAK & DRI VEN, D I G I T I ZED & \\
\hline 2118 & $\sigma$ & 5.379 & 2.759 & .104 & .064 & - 92 & 900 & TH I CENESS & ( CAII PER), & EMVEC $\theta$, & METER & DRIVEN & \\
\hline L58 1 & $\boldsymbol{\theta}$ & 5.380 & 2.830 & .152 & -.011 & .62 & $90 \mathrm{I}$ & THICKNESS & ( CAII PER), & IMI. & MATOR & DRI VEN & \\
\hline
\end{tabular}


RE PORT N.. $58 G$

IAPPI COLLABERATIVE REFERENCE PREGRAM

ANALYSIS T90-1 TABLE 2

THICENESS (CAIIPER), THOUSANDIHS OF AN INCH

IAPPI STANDARD IAII $19-76$

\begin{tabular}{|c|c|c|c|}
\hline \multirow{2}{*}{$\begin{array}{l}\mathrm{LAB} \\
C \in D E\end{array}$} & \multirow[b]{2}{*}{ F } & \multicolumn{2}{|c|}{ MEANS } \\
\hline & & J64 & J83 \\
\hline 1153 & $*$ & 5.388 & 2.937 \\
\hline 1442 & $\theta$ & 5.399 & 2.879 \\
\hline I $242 P$ & - & 5.406 & 2.825 \\
\hline L1 00 & $\theta$ & 5.409 & 2.806 \\
\hline L390 & $\boldsymbol{\theta}$ & 5.452 & 2.880 \\
\hline L233 & $\mathrm{x}$ & 5.579 & 2.753 \\
\hline 1259 & $\mathbf{x}$ & 10.121 & 2.734 \\
\hline L396 M & - & 51.200 & 26.400 \\
\hline
\end{tabular}

GMEANS: $\quad 5.259 \quad 2.738$ S5\% EILIPSE:
CEARDINATES YAJEE MINOR

\section{.229 .063}

$.199 \quad .013$

$.168 \quad-.032$

.1580 .048

$.250 \quad-.201$

$3.633-3.231$

$50.065-12.806$
$.239-.022$

.293 .101 $\triangle$ VO

R.SDR VAR PREPERTY---IEST INSTRUMENT---CONDITIENS

1.11901 THICRNESS (CAIIPER), TMI.

$.8790 V$ THICKNESS (CAIIPER), TMI.

-79 9OP IHICXNESS (CAIIPER), NESSMER,

MGTEE DRIVEN

METOR DRIVEN, DIGITIZED

-85 90V THICKNESS (CAIIPER), TMI,

METER DRIVEN, ISE RS34

$.9890 T$ THICKNESS (CALIPER), TKI.

MEIOR DRI VBN

-8890Q THICKNESS (CALIPER), EKVECE, METOR DRIVEN $169.6790 T$ THICKNESS (CAIIPER), THI. METER DRIVEN 15.40 90S THICENESS (CAIIPER); SCHEPPER, HAND DRIVEN

1.00
WITH GAMMA = 41 DEgREES 
THICKNESS (CALIPER)

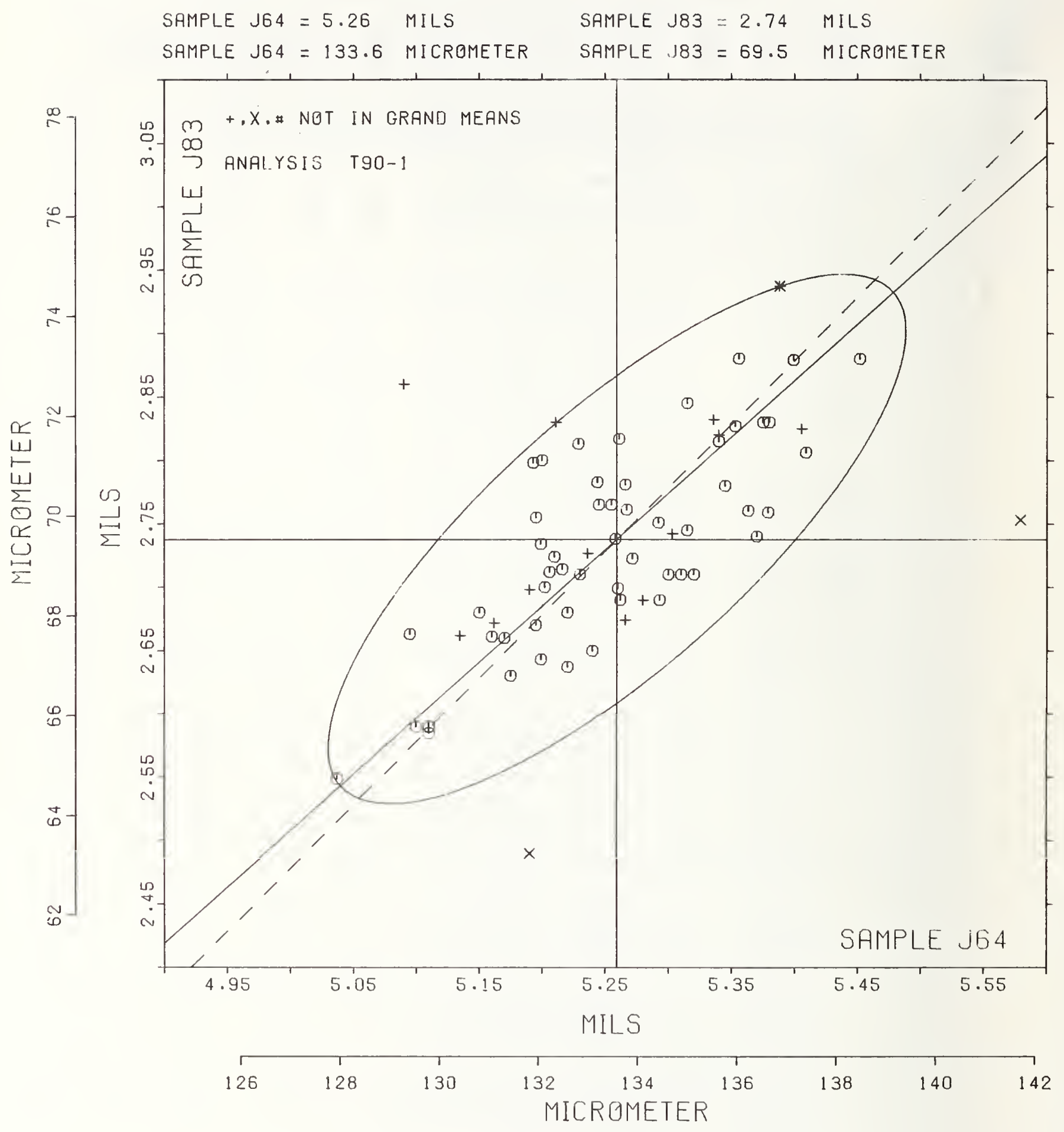


TAPPI STANDARD T410 OS-68

\begin{tabular}{|c|c|c|c|c|c|c|c|c|c|c|c|c|c|}
\hline \multirow{3}{*}{$\begin{array}{l}\text { LAB } \\
\text { CADE }\end{array}$} & \multirow{3}{*}{$\begin{array}{c}\text { SAMPIE } \\
\text { D32 } \\
\text { NEAN }\end{array}$} & \multicolumn{4}{|c|}{ BEND } & \multirow{3}{*}{$\begin{array}{c}\text { SAMPIE } \\
\text { D33 } \\
\text { MEAN }\end{array}$} & \multicolumn{4}{|c|}{ MANIIA ENVELGPE } & \multirow{2}{*}{ TEST } & \multirow[t]{2}{*}{$\mathrm{D}_{\bullet}=$} & \multirow[t]{2}{*}{10} \\
\hline & & $79 \mathrm{GE}$ & AS PEF & SQUARE MI & & & $118 \mathrm{GR}$ & MS PER & SQUARE ME & & & & \\
\hline & & DEV & $\mathrm{N}, \mathrm{DEV}$ & SDR & R.SDR & & DEV & $\mathrm{N}, \mathrm{DEV}$ & SDR & R. SDR & VAR & $\mathbf{F}$ & $I A B$ \\
\hline 1100 & $79 \cdot 30$ & .02 & .04 & .31 & .89 & 118.10 & .30 & .32 & 1.52 & 1.19 & $95 \mathrm{C}$ & $\theta$ & I 100 \\
\hline 1121 & $79 \cdot 31$ & .03 & .05 & .48 & 1.41 & $116 \cdot 31$ & -1.49 & -1.58 & 1.21 & .95 & 953 & $\theta$ & 1121 \\
\hline L162 & 79.00 &. .28 &. .50 & .00 & .00 & 116.59 & -1.21 & -1.28 & 1.77 & 1.38 & $95 \mathrm{~K}$ & $\theta$ & L 162 \\
\hline L213 & 80.20 & .92 & 1.65 & .44 & 1.27 & 118.28 & .48 & .51 & 1.65 & 1.29 & $95 \mathrm{~F}$ & ह & I2 13 \\
\hline I233 & 30.43 & -48.85 & -87.72 & .19 & .57 & $45 \cdot 35$ & -72.46 & -76.70 & .45 & .35 & $95 \mathrm{~T}$ & $*$ & L233 \\
\hline L249 & 79.46 & .18 & .32 & .30 & .87 & 118.80 & 1.00 & 1.06 & 1.19 & .92 & $95 I$ & 6 & 1249 \\
\hline L280 & 79.14 & .14 &. .25 & .46 & 1.33 & 117.97 & .17 & .18 & 1.51 & 1.18 & $95 T$ & $\theta$ & L 280 \\
\hline 1305 & 78.40 & -.88 & -1.58 & .28 & .82 & 116.30 & -1.50 & -1.59 & .89 & .69 & $95 T$ & $\theta$ & I305 \\
\hline 1339 & 78.51 &. .77 & -1.38 & .19 & .54 & 117.37 &. .43 & -.46 & .69 & .54 & $95 \mathrm{~T}$ & $\theta$ & L339 \\
\hline 1342 & 20.39 & 1.11 & 2.00 & .34 & 1.00 & 118.35 & .55 & .58 & 1.78 & 1.39 & $95 \mathrm{C}$ & $\sigma$ & L342 \\
\hline L34.4 & 79.89 & .61 & 1.09 & .33 & .97 & 118.41 & .61 & .64 & .21 & .16 & S5T & $\sigma$ & I344 \\
\hline 1442 & $75 \cdot 16$ & -.12 & -.22 & .16 & .48 & 117.71 & -.09 & -10 & .65 & .50 & $95 \mathrm{~K}$ & $\theta$ & 1442 \\
\hline 1557 & 78.91 & -.37 & -.66 & .59 & 1.71 & 116.53 & -1.27 & $-1 \cdot 34$ & 1.72 & 1.34 & $95 \mathrm{C}$ & $\theta$ & L557 \\
\hline L559 & 79.43 & .15 & .26 & .44 & 1.27 & 118.15 & .35 & .37 & 1.89 & 1.47 & $95 \mathrm{~K}$ & $\theta$ & L559 \\
\hline L56 7 & 79.59 & .31 & .56 & .43 & 1.26 & 118.26 & .46 & .48 & 1.67 & 1.30 & $95 E$ & e & L567 \\
\hline 1574 & $78 \cdot 75$ & -.53 &. .95 & .37 & 1.08 & 117.41 & -.39 & -.41 & 1.52 & 1.18 & S5D & 6 & 1574 \\
\hline I597 & $80 \cdot 26$ & .98 & 1.76 & .76 & 2.21 & 123.00 & 5.20 & 5.50 & 3.16 & 2.47 & $95 \mathrm{C}$ & \# & 1597 \\
\hline I626 & 78.65 & -.63 & -1.13 & .40 & 1.16 & 117.35 & -.45 & .048 & 1.14 & .89 & $95 \mathrm{E}$ & $\theta$ & I6 26 \\
\hline 2688 & 79.09 & .19 &. .33 & .30 & .89 & 118.82 & 1.02 & 1.07 & 1.25 & .97 & $95 T$ & $\boldsymbol{\sigma}$ & 1688 \\
\hline L693 & 79.86 & .58 & 1.04 & .36 & 1.04 & 119.72 & 1.92 & 2.03 & .83 & .65 & $95 G$ & $\theta$ & L693 \\
\hline
\end{tabular}

GR. MEAN $=79.28 \mathrm{G} / \mathrm{SC} . \mathrm{METER}$ SD MEANS - .56 G/SQ. METER
GRAND MEAN $=117.80$ G/SQ. METER

SD GF MEANS $=.94 \mathrm{G} /$ SQ.METER

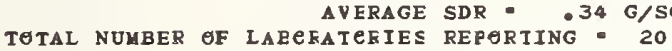

Best values: D32 $79.2 \pm 0.9$ grams per square meter

D.33 $117.8 \pm 1.5$ grams per square meter

The following laboratories were omitted from the

grand means because of extreme test results: 597.

Data from the following laboratories apppear to be

off by a multiplicative factor: 233 .

REPERT NO, 586

\begin{tabular}{|c|c|c|c|}
\hline \multicolumn{2}{|l|}{ LAB } & \multicolumn{2}{|c|}{ MEANS } \\
\hline CODE & $\boldsymbol{F}$ & D32 & D33 \\
\hline 2233 & * & 30.43 & $45 \cdot 35$ \\
\hline 1305 & $\theta$ & 78.40 & 116.30 \\
\hline 1339 & $\sigma$ & 78.51 & 117.37 \\
\hline 1626 & $\theta$ & 78.65 & 117.35 \\
\hline 1574 & $\theta$ & 78.75 & 117.41 \\
\hline 1557 & $\theta$ & 78.91 & 116.53 \\
\hline 2162 & $\theta$ & 79.00 & 116.59 \\
\hline 2688 & $\sigma$ & 79.09 & 118.82 \\
\hline L280 & $\theta$ & 79.14 & 117.97 \\
\hline 1442 & $\theta$ & 79.16 & 117.71 \\
\hline 2100 & $\theta$ & 79.30 & 118.10 \\
\hline L1 21 & $\boldsymbol{\theta}$ & 79.31 & 116.31 \\
\hline L559 & $\boldsymbol{\sigma}$ & 79.43 & 118.15 \\
\hline L249 & $\theta$ & 79.46 & 118.80 \\
\hline L56 7 & $\theta$ & 79.59 & 118.26 \\
\hline 2693 & $\theta$ & 79.86 & 119.72 \\
\hline 2344 & $\theta$ & 79.89 & 118.41 \\
\hline L213 & $\theta$ & 80.20 & 118.28 \\
\hline L5ऽ 7 & * & 80.26 & 123.00 \\
\hline L342 & o & 80.39 & 118.35 \\
\hline & & $\begin{array}{c}79.28 \\
95 \%\end{array}$ & $\begin{array}{l}117.80 \\
\text { IIIPSE: }\end{array}$ \\
\hline
\end{tabular}

TAPPI COIIABERATIVE REFERENCE PREGRAM ANALYSIS T95-1 TABIE 2 GRAMMAGE (MASS PER UNII AREA)

TAPPI STANDARD T 40 OS=68

CEORDINATES AVG

MAJER MINER R.SDR VAR PREPERTY---TEST INSTRUMENT-=CONDITIONS

$-86.14 \quad 14.69$

1.73

$-.71$

.67

.038

$-1.31 \quad-19$

$-1.22 \quad-.24$

$85 \quad .59$

.10 .20

0.13

.28 .10

$-1.35$

.38

$.98 \quad .25$

$.54 \quad 0.09$

1.99

-80

.064

1.14 .25

$.96 \quad-.79$

2.841 .11

.46 95T BASIS WEIGHT (GRAMMAGE). TEMPLATE CUT

.76 95T BASIS WEIGHT (GRAMMAGE); TEMPLATE CUT

.54 95T BASIS WEIGHT (GRAMMAGE); TEMPLATE CUT

1.02 95E BASIS WEIGHT (GRAMMAGE), GUILIOTINE TYPE CUTTER

1.13 95D BASIS WEIGHT (GRAMMAGE), DIE CUT

1.53 95C BASIS WEIGHT (GRAMMAGE), CUTTING BEARD

$.6995 \mathrm{~K}$ BASIS WEIGHT (GRAMMAGE), WEIGHED AS RECEIVED

- 33 95T BASIS WEIGHT (GRAMMAGE), TEMPIATE CUT

1. 25 95T BASIS WEIGHT (GRAMNAGE), TEMPLATE CUT

-49 95E BASIS WEIGHT (GRAMUAGE); WEIGBED AS RECEIVED

1.04 G5C BASIS WEIGHT (GRAKMAGE), CUTTING BeARD

1.13 ९5B BASIS WEIGHT (GRAMMAGE), CENCERA CUTTER

1.37 95K BASIS WEIGHT (GRAMMAGE); WEIGHED AS RECEIVED

-90 95I BASIS TEIGHT (GRAMMAGE), INGENTO PAPER CUTTER

1.28 95E BASIS WEIGHT (GRAMMAGE), GUILIGTINE TYPE CUTTER

.84 95G BASIS WEIGHT (GRAMMAGE), PRECISION CUTTER

.57 95T BASIS TEIGBT (GRAMMAGE), TEMPLATE CUT

1. 28 95F BASIS WEIGHT (GRAMMAGE), FOUR-SOUARE CUTTER

2.34 95C BASIS WEIGHT (GRAM(MAGE), CUTTING BEARD

1.19 95C BASIS WEIGHT (GRAMMAGE), CUTTING BGABD

1.00

WITH GAMMA - 65 DEGREES
TEST DETERMINATIONS = 10

18 IABS IN GRAND MEANS AVERAGE SDR $=1.28 \mathrm{G} /$ SQ.METER 


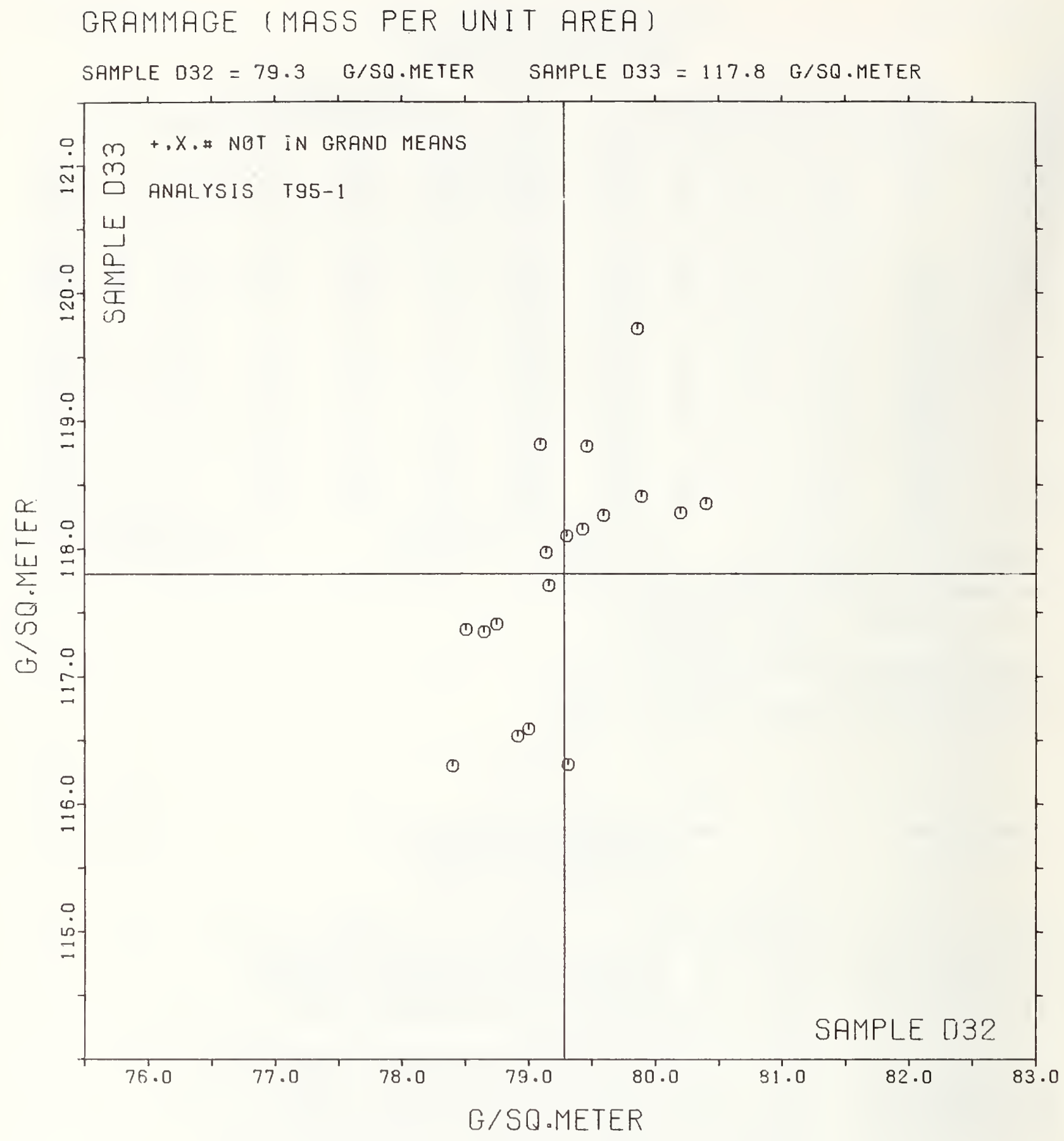


SUMNARY TABLE

\begin{tabular}{|c|c|c|c|c|c|c|c|c|c|c|}
\hline TEST METBOD & $\begin{array}{l}\text { SAMPLE } \\
\text { CODE }\end{array}$ & $\begin{array}{l}\text { GRAND } \\
\text { MEAN }\end{array}$ & $\begin{array}{l}\text { SD EF } \\
\text { MEAN }\end{array}$ & $\begin{array}{l}\text { AVER } \\
\text { SDR }\end{array}$ & $\begin{array}{l}\text { REPL } \\
\text { CRP }\end{array}$ & $\begin{array}{l}\text { LABS } \\
\text { INCL }\end{array}$ & $\begin{array}{l}\text { LABS } \\
\text { PARTIC }\end{array}$ & $\begin{array}{l}\text { REPL } \\
\text { TAPPI }\end{array}$ & REPEAT & REPROD \\
\hline AIR RESISTANCE, GURLEY & 821 & $51 \cdot 2$ & 3.0 & 4.3 & 10 & 55 & 60 & 10 & 3.8 & 8.3 \\
\hline T40-1 GURIEY UNITS & $\mathrm{K} 43$ & 16.4 & .8 & 1.2 & & & & & 1.0 & $2 \cdot 3$ \\
\hline $\begin{array}{ll}\text { AIR RESISTANCE, } & \text { SHEFFIELD } \\
\text { T40-2 } & \text { SHEFF. UNIT }\end{array}$ & $\begin{array}{l}821 \\
\times 43\end{array}$ & $\begin{array}{r}71.1 \\
167.6\end{array}$ & $\begin{array}{l}5 \cdot 9 \\
9.2\end{array}$ & $\begin{array}{l}5.9 \\
8.8\end{array}$ & 10 & 40 & 45 & 10 & $\begin{array}{l}5.2 \\
7.7\end{array}$ & $\begin{array}{l}16 \cdot 4 \\
25 \cdot 5\end{array}$ \\
\hline $\begin{array}{ll}\text { AIR RESISTANCE, GURLEY HG FLOTATION } \\
\text { T } 41-1 & \text { SEC } / 10 \text { CC }\end{array}$ & $\begin{array}{l}E 68 \\
E 65\end{array}$ & 651 & $\begin{array}{l}62 . \\
63\end{array}$ & $\begin{array}{r}85 . \\
148 .\end{array}$ & 10 & 12 & 12 & 10 & $\begin{array}{r}75 . \\
129\end{array}$ & $\begin{array}{l}171 . \\
173 .\end{array}$ \\
\hline $\begin{array}{l}\text { SNOETHNESS, PARKER PEINTSURF } \\
\text { T44-1 }\end{array}$ & $\begin{array}{r}\mathbf{1} 45 \\
-183\end{array}$ & 5.47 & .43 & $\begin{array}{r}10 \\
.24\end{array}$ & 10 & 9 & 9 & 10 & .09 & 1.20 \\
\hline $\begin{array}{l}\text { SMEबTHNESS, SHEFFIEID } \\
\text { T45-1 SHEFF, CNITS }\end{array}$ & $\begin{array}{l}\mathbf{B} 45 \\
183\end{array}$ & $\begin{array}{l}160 \cdot 3 \\
105 \cdot 4\end{array}$ & $\begin{array}{l}7.0 \\
6.8\end{array}$ & $\begin{array}{r}9.2 \\
10.7\end{array}$ & 15 & 89 & 91 & 10 & $\begin{array}{l}8.0 \\
9.4\end{array}$ & $\begin{array}{l}20.0 \\
19.6\end{array}$ \\
\hline $\begin{array}{l}\text { SUOӨTHNESS, HEKK } \\
\text { T45-2 HEKR SECONDS }\end{array}$ & $\begin{array}{l}\mathbf{8} 45 \\
\mathbf{8 8 3}\end{array}$ & $\begin{array}{l}30.2 \\
54.9\end{array}$ & $\begin{array}{l}3.9 \\
7.6\end{array}$ & $\begin{array}{l}2.6 \\
9.2\end{array}$ & 15 & 9 & 11 & 10 & $\begin{array}{l}2.3 \\
8.1\end{array}$ & $\begin{array}{l}10.9 \\
21.5\end{array}$ \\
\hline $\begin{array}{lr}\text { SMEOTHNESS, } & \text { HENDTSEN } \\
\text { T47-1 } & \text { ML } / M I N\end{array}$ & $\begin{array}{l}\text { B 45 } \\
\Delta 83\end{array}$ & $\begin{array}{l}203 . \\
113\end{array}$ & 14. & $\begin{array}{l}19 . \\
18 .\end{array}$ & 10 & 8 & 8 & 10 & $\begin{array}{l}16 . \\
16 .\end{array}$ & $\begin{array}{ll}40 . \\
36 .\end{array}$ \\
\hline $\begin{array}{l}\text { R \& N INB ABSERPTION } \\
\text { T56-1 } \\
\text { K \& N UNITS }\end{array}$ & $\begin{array}{l}E 48 \\
\text { H58 }\end{array}$ & $\begin{array}{l}24.7 \\
61.4\end{array}$ & $\begin{array}{l}3.3 \\
6.9\end{array}$ & $: 8$ & 4 & 8 & 11 & 4 & $\begin{array}{l}1.2 \\
1.0\end{array}$ & $\begin{array}{r}9.0 \\
19.2\end{array}$ \\
\hline $\begin{array}{l}\text { PH, } C O L D \\
\text { T57-1 }\end{array}$ & $\begin{array}{l}\text { J78 } \\
\text { J } 75\end{array}$ & $\begin{array}{l}7.339 \\
6.189\end{array}$ & $\begin{array}{l}.143 \\
.226\end{array}$ & $\begin{array}{r}.043 \\
.029\end{array}$ & 5 & 5 & 6 & 2 & $\begin{array}{l}.083 \\
.056\end{array}$ & $\begin{array}{l}.402 \\
.629\end{array}$ \\
\hline $\begin{array}{l}\text { PH, HबT } \\
\text { T57-2 }\end{array}$ & $\begin{array}{l}\mathbf{J} 78 \\
\mathbf{J} 75\end{array}$ & $\begin{array}{l}7.914 \\
5.841\end{array}$ & $\begin{array}{l}.208 \\
.395\end{array}$ & .035 & 5 & 4 & 4 & 2 & $\begin{array}{l}.069 \\
.069\end{array}$ & $\begin{array}{r}.579 \\
1.095\end{array}$ \\
\hline $\begin{array}{l}\text { OPACITY, HAL TYPE, 89\% EACKING } \\
\text { T60-1 }\end{array}$ & $\begin{array}{l}\text { E } 86 \\
\text { H21 }\end{array}$ & $\begin{array}{l}89 \cdot 75 \\
72.36\end{array}$ & $\stackrel{.54}{1.00}$ & $\because 35$ & 10 & 78 & 89 & 5 & $\begin{array}{r}.44 \\
1.26\end{array}$ & $\begin{array}{l}1.52 \\
2.90\end{array}$ \\
\hline $\begin{array}{l}\text { APACITY, H\&L TYPE, PAPEE HACEING } \\
\text { T60-2 } \\
\text { PERCENT }\end{array}$ & $\begin{array}{l}\text { E86 } \\
\text { H2 } 1\end{array}$ & $\begin{array}{l}91.49 \\
75.61\end{array}$ & $\begin{array}{l}.28 \\
.27\end{array}$ & $\begin{array}{r}.30 \\
1.10\end{array}$ & 10 & 6 & 6 & 5 & $\begin{array}{r}\cdot 37 \\
1 \cdot 36\end{array}$ & $\begin{array}{r}.81 \\
1.23\end{array}$ \\
\hline $\begin{array}{l}\text { OPACITY, ELREPEO TYPE, PAPER BACEING } \\
\text { T60-3 } \\
\text { PERCENT }\end{array}$ & $\begin{array}{l}E 86 \\
821\end{array}$ & $\begin{array}{l}92.07 \\
77.08\end{array}$ & .19 & $\begin{array}{l}.19 \\
.76\end{array}$ & 10 & 14 & 16 & 5 & .24 & $\begin{array}{r}.56 \\
1.94\end{array}$ \\
\hline $\begin{array}{l}\text { HLUE REFLECTANCE, DIRECTIONAL } \\
\text { T65-1 PERCENT }\end{array}$ & $\begin{array}{l}\mathbf{J} 36 \\
\mathbf{J} 79\end{array}$ & $\begin{array}{l}83 \cdot 93 \\
68 \cdot 14\end{array}$ & $\begin{array}{r}.60 \\
. \\
.35\end{array}$ & $\begin{array}{l}.12 \\
.22\end{array}$ & 8 & 21 & 46 & 6 & .14 & $\begin{array}{r}1.66 \\
\cdot 98\end{array}$ \\
\hline $\begin{array}{l}\text { HLUE REFLECTANCE, DIFFUSE, WITH TRAP } \\
\text { T65-2 }\end{array}$ & $\begin{array}{l}\text { J36 } \\
\text { J79 }\end{array}$ & $\begin{array}{l}84.45 \\
67.87\end{array}$ & .47 & $\begin{array}{l}.08 \\
.18\end{array}$ & 8 & 18 & 19 & 6 & $\begin{array}{l}.10 \\
.20\end{array}$ & $\begin{array}{l}1.31 \\
2.90\end{array}$ \\
\hline $\begin{array}{l}\text { HLUE REFLECTANCE, DIFFUSE, NO TRAP } \\
\text { T65-3 PERCENT }\end{array}$ & $\begin{array}{l}\text { J } 36 \\
\text { J } 79\end{array}$ & $\begin{array}{l}84.05 \\
68 \cdot 47\end{array}$ & .61 & .08 & 8 & 13 & 16 & 6 & .09 & $\begin{array}{l}1.69 \\
1.81\end{array}$ \\
\hline $\begin{array}{ll}\text { SPECULAR GLESS, } 75 \text { DEGREE } \\
\text { T75-1 } & \text { GLOSS UNIIS }\end{array}$ & $\begin{array}{l}\text { E87 } \\
\text { E51 }\end{array}$ & $\begin{array}{l}68.3 \\
68.1\end{array}$ & 1.7 & $\begin{array}{l}1.9 \\
1.1\end{array}$ & 10 & 49 & 54 & 5 & $\begin{array}{l}2.4 \\
1.4\end{array}$ & $\begin{array}{l}5.0 \\
4.4\end{array}$ \\
\hline $\begin{array}{l}\text { THI CENESS (CAIIPER) } \\
\text { T90-1 MILS }\end{array}$ & $\begin{array}{l}\sqrt{64} \\
\mathrm{~J} 83\end{array}$ & $\begin{array}{l}5.259 \\
2.738\end{array}$ & $\begin{array}{l}.090 \\
.082\end{array}$ & .044 & 10 & 55 & 73 & 10 & $\begin{array}{l}.039 \\
.035\end{array}$ & $\begin{array}{l}.250 \\
.227\end{array}$ \\
\hline $\begin{array}{l}\text { GRAMNAGE (NASS PER UNIT AREA) } \\
\text { T95-1 } \\
\text { G/SG.METER }\end{array}$ & $\begin{array}{l}\text { D32 } \\
\text { D33 }\end{array}$ & $\begin{array}{r}79.28 \\
117.80\end{array}$ & .56 & $\begin{array}{r}.34 \\
1.28\end{array}$ & 10 & 18 & 20 & 3 & $\begin{array}{r}.55 \\
2.05\end{array}$ & $\begin{array}{l}1.61 \\
3.13\end{array}$ \\
\hline
\end{tabular}


NBS-114A (REV. 9-78)

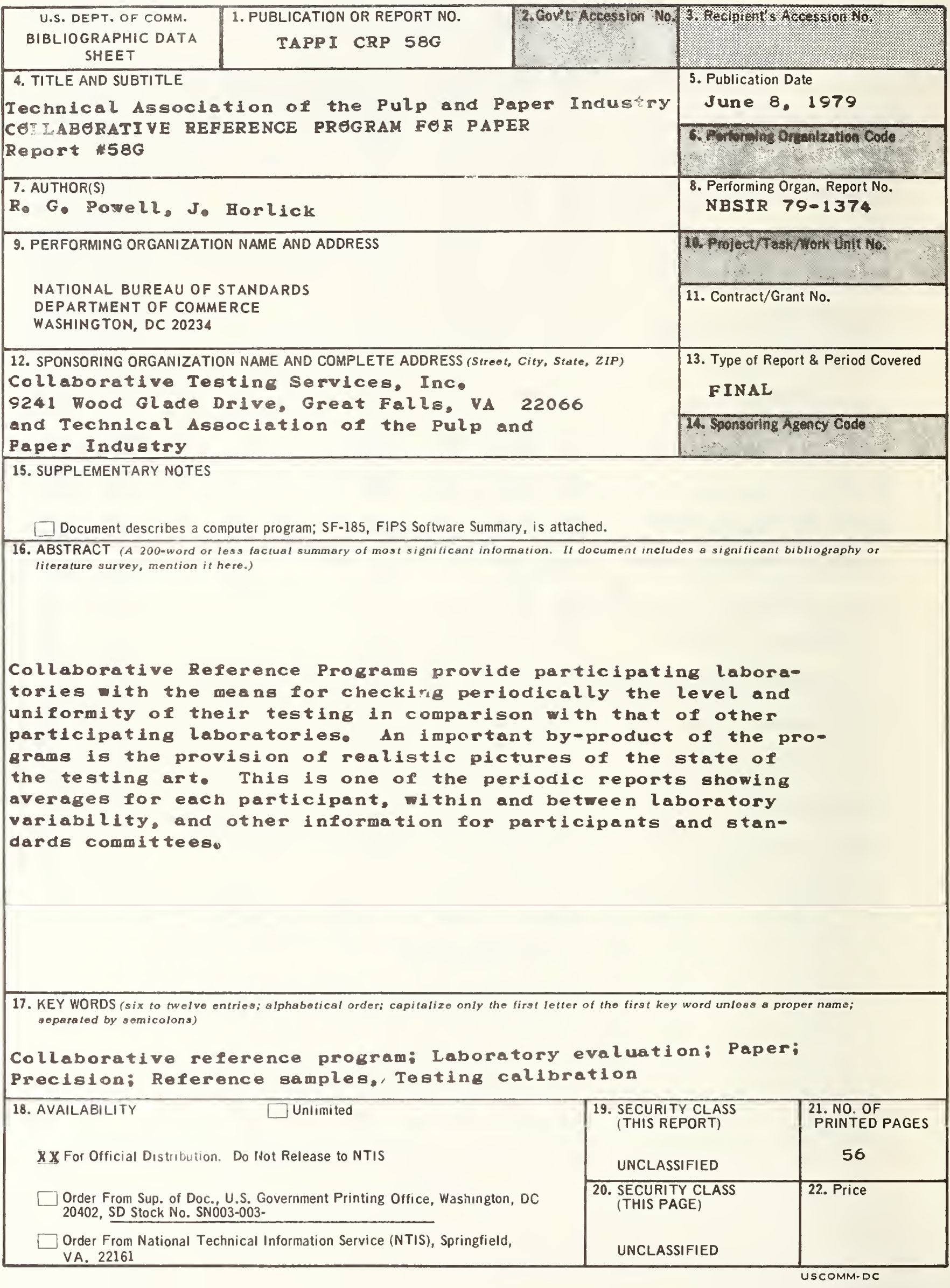


